\title{
Behavioural and physiological ecology of coastal marine fish:
}

\section{basic and applied perspectives}

by

Jacob W. Brownscombe

A thesis submitted to the Faculty of Graduate and Postdoctoral Affairs in partial fulfillment of the requirements for the degree of

Doctor of Philosophy

in

Biology

Carleton University

Ottawa, Ontario

(C) 2016

Jacob W. Brownscombe 


\begin{abstract}
Energy is the currency of life, by which we can measure how ecological and anthropogenic factors influence individual fitness, scaling up to population and ecosystem dynamics. Energy is expended and gained by organisms through diverse behavioural tactics aimed at maximizing fitness. My overarching hypothesis for this dissertation is that ecological and anthropogenic factors influence animal behaviour and energetics. I tested this hypothesis in two coastal marine fish species, bonefish (Albula vulpes) and great barracuda (Sphyraena barracuda) using a combination of field studies and controlled experiments. In the wild, landscape features had the greatest impact on bonefish activity and energy expenditure at both fine (i.e., between habitats on a single coral reef crest) and broad (i.e., between coastal habitats and regions) spatial scales. Diel period, water temperature, and tide state also influenced bonefish behaviour and energetics, with some consistent patterns across environments, including greater activity and energy expenditure during the day, as well as ebbing and low tides. Bonefish activity levels and habitat selection also corresponded with temperature-related physiological performance. Comparing two disparate coastal ecogeographic regions, activity- and temperature-based estimates of bonefish energy expenditure were higher in the fringing coral reefs of tropical Culebra, Puerto Rico than the expansive sand flats of sub-tropical Eleuthera, The Bahamas; however, home ranges were significantly larger in Eleuthera than Culebra, which likely has significant energetic costs that may contribute to differences in growth rates between the regions. From a more applied perspective, a common anthropogenic stressor, recreational angling, caused significant locomotory (i.e.,
\end{abstract}


swimming capabilities) and behavioural (i.e., refuge use) impairment in bonefish and great barracuda, which resulted in increased post-release predation risk. Retaining bonefish for a short period prior to release reduced this impairment and may be a useful strategy for improving post-release survival in environments with high predator burden. Collectively, by examining how ecological and anthropogenic factors influence fish behaviour and energetics, my dissertation has advanced our understanding of fundamental ecology and management of coastal marine fish and their ecosystems. 


\section{Acknowledgements}

First and foremost, I am grateful to my thesis advisors Steven Cooke and Andy Danylchuk, who have provided me the utmost support and contributed significantly to my development into the person and scientist I am today. The level of opportunity, moral and financial support (as well as a few beers and blended mojitos after work) they have provided me is truly remarkable. They have taught me not only the fundamentals of science, but also the value and skills of social networking, extracurricular activities, science outreach, striking a healthy work-life balance, and how to acquire research funding and scholarships. Since the beginning they treated me not simply as a student, but as a colleague, and their faith in my abilities has strengthened my confidence as a scientist, enabling me to accomplish far more than I could have ever imagined in these past 4 years.

I also extend my gratitude to my thesis committee members, Sue Bertram and Pat Walsh, as well as my comprehensive exam external Gabriel Blouin-Demers and dissertation defence examiners Nann Fangue and Murray Richardson, who contributed positive feedback and constructive criticism that helped shape this thesis and contributed to my development as a scientist. They are all busy people, but still took the time to provide critical input to my work while asking for nothing in return, for which I am very thankful.

Thank you my partner Caitlin Higginson and my entire family for their unconditional support. I would not do the work that I do had I not grown up fishing and 
exploring the wilderness with my father and grandfather. My mother imparted in me a level of determination that is certainly required to spend 10 years in post secondary education. Without the support of Caitlin, there is no way I would have been as productive as have been in these past years. She has kept me grounded with a life outside of work, with tireless tolerance of my frequent absence while on research trips, and my general level of grumpy cynicism.

Being a part of both the Cooke and Danylchuk research labs, I have been fortunate to work with many remarkable young scientists. In particular, I owe a huge thanks to Lee Gutowsky; without him I would not have done this Ph.D. He has been one of my greatest mentors, collaborators, and friends. I owe thanks to Jason Thiem, Graham Raby, Jacqueline Chapman, Taylor Ward, Alex Wilson, Charles Hatry, Nick Lapointe, Liane Nowell, Robert Lennox, Shannon Bower, Emma Samson, Petra Szekeres, Dirk Algera, Jordann Foster, Aaron Zolderdo, Mike Lawrence, Vivian Nguyen, Jill Brookes, Lucas Griffin, Tyler Gagne, Chris Haak, Sarah Becker, Karen Murchie, and many others for contributing to my research and development as a scientist. Thanks also to the numerous staff at the Cape Eleuthera Institute, especially Aaron Shultz, Ed Brooks, and Zach Zuckerman for supporting my research in Eleuthera. Thanks to Craig Lilyestrom, Ricardo Colón-Merced, Capt. Chris Goldmark, Sammy Hernandez, Zorida Mendez, Todd and Shellie Plaia, and Henry Cruz for support in Culebra. Thanks to Jason Thiem, Adrian Gleiss, and Ken Sakamoto for helping me learn how to analyze accelerometry data. 
During my Ph.D. I have received financial support including a Natural Sciences and Engineering Research Council (NSERC) PGS-D3 scholarship, American Fisheries Society Steven Berkeley Marine Conservation Fellowship, Fisheries Society of the British Isles Travel Grant, Ontario Federation of Anglers and Hunters Research Grant, Carleton University Graduate Research and Innovation Thinking Grant, American Fisheries Society CARS Clemens-Rigler Travel Award, International Bio-logging Symposium Student Travel Award, Carleton University Travel Award, Peter A. Larkin Award for Excellence in Fisheries, and a Research Assistantship from Carleton University. Research funding was provided by NSERC Discovery Grants (awarded to Cooke), the University of Puerto Rico Sea Grant Program (awarded to Danylchuk), and Bonefish and Tarpon Trust. Thanks also to Temple Fork Outfitters, RIO Products, Costa Del Mar, Patagonia, and Umpqua Feather Merchants for their in-kind support. 


\section{Thesis Format and Co-authorship}

This dissertation contains five data chapters written in manuscript format, which are at various stages of the publication process in peer -reviewed journals. For this reason there is some repetition between chapters to remain consistent with my contributions to the scientific literature. While this dissertation contains my research, many coauthors contributed to the data chapters. Here I list my contributions to each chapter, as well as that of my coauthors.

\section{Chapter 2. Foraging behaviour and activity of a marine benthivorous fish estimated using tri-axial accelerometer biologgers}

Brownscombe, J. W., Gutowsky, L. F., Danylchuk, A. J., \& Cooke, S. J. 2014. Foraging behaviour and activity of a marine benthivorous fish estimated using tri-axial accelerometer biologgers. Marine Ecology Progress Series, 505, 241-251.

I designed this study, conducted the data collection, data analysis, and manuscript preparation. Gutowsky contributed to data analysis and manuscript preparation. Danylchuk, and Cooke contributed to study design and manuscript preparation. 


\section{Chapter 3. Spatiotemporal drivers of energy expenditure in a coastal marine fish}

Brownscombe, J. W., Cooke, S. J., \& Danylchuk, A. J. In Review. Spatiotemporal drivers of energy expenditure in a coastal marine fish. Oecologia. OECO-D-16-00080

I designed the research project, conducted experiments and field studies, analyzed the data and wrote the manuscript. Danylchuk and Cooke contributed to research design and manuscript preparation

\section{Chapter 4. Ecological energetics of a marine teleost fish across ecogeographic regions}

Brownscombe, J. W., Cooke, S. J., Murchie, K. J., \& Danylchuk, A. J. In Prep. Ecological energetics of a marine teleost fish across ecogeographic regions. Ecography.

I designed the research project, conducted experiments and field studies, analyzed the data and wrote the manuscript. Murchie conducted field studies and provided edits to the manuscript. Danylchuk and Cooke contributed to study design and manuscript preparation. 


\section{Chapter 5. Recovery bags reduce post-release impairments in locomotory activity and behaviour of bonefish following exposure to angling-related stressors}

Brownscombe, J. W., Thiem, J. D., Hatry, C., Cull, F., Haak, C. R., Danylchuk, A. J., \&

Cooke, S. J. 2013. Recovery bags reduce post-release impairments in locomotory activity and behavior of bonefish (Albula spp.) following exposure to angling-related stressors. Journal of Experimental Marine Biology and Ecology, 440, 207-215.

I developed the study design, conducted field work, data analysis and wrote the manuscript. Thiem contributed to the experimental design, experimentation, data analysis, and manuscript editing. Hatry, Cull, and Haak assisted in experimentation, and edited the manuscript. Cooke contributed to the experimental design, conducted experiments, and edited the manuscript. Danylchuk contributed to the experimental design and edited the manuscript. 
Chapter 6. Fishing-related stressors inhibit refuge-seeking behaviour in released sub-adult great barracuda

Brownscombe, J. W., Nowell, L., Samson, E., Danylchuk, A. J., \& Cooke, S. J. 2014. Fishing-related stressors inhibit refuge-seeking behavior in released subadult great barracuda. Transactions of the American Fisheries Society, 143, 613-617.

I designed the experiments, conducted data analysis and wrote the manuscript. Nowell and Samson contributed to data collection and manuscript preparation. Danylchuk and Cooke contributed to experimental design and manuscript preparation. 
Table of Contents

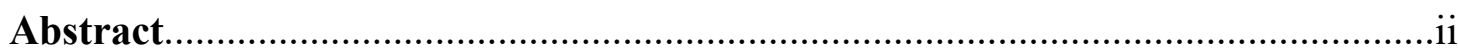

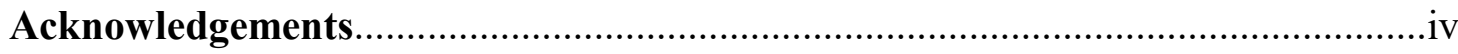

Thesis Format and Co-authorship............................................................... vii

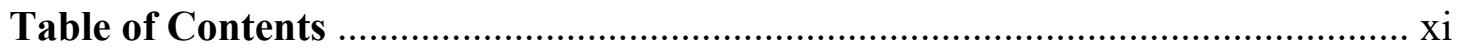

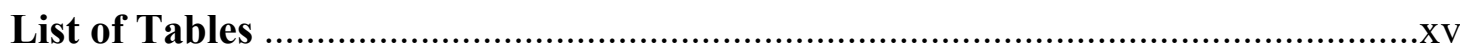

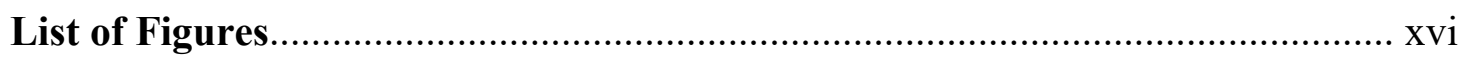

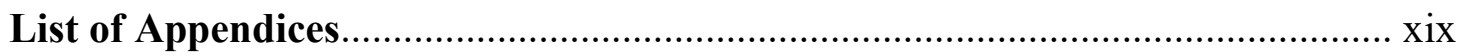

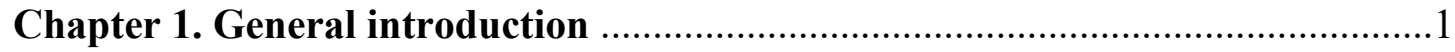

1.1 The basics - Animal behaviour and energetics............................................ 1

1.2 Applied conservation - Anthropogenic impacts......................................5

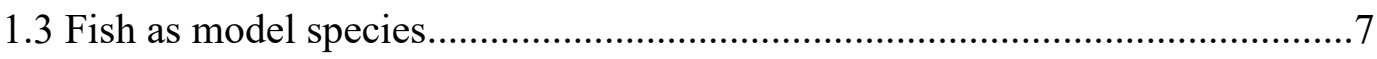

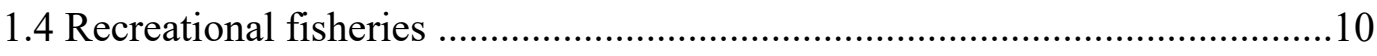

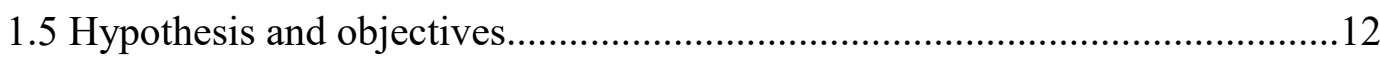

Chapter 2. Foraging behaviour and activity of a marine benthivorous fish estimated using tri-axial accelerometer biologgers..............................................13

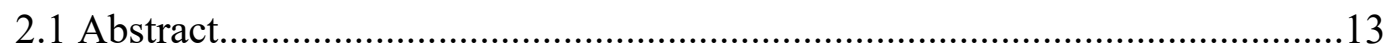

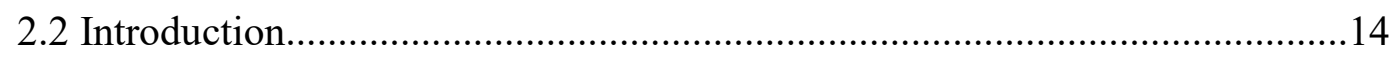

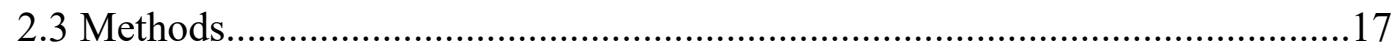

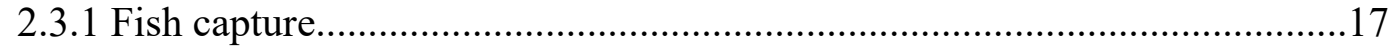

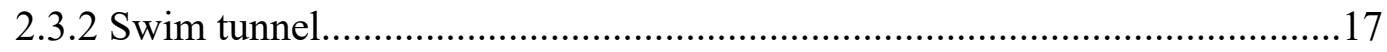

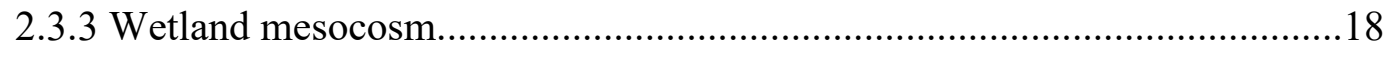

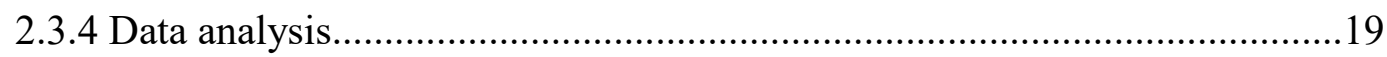




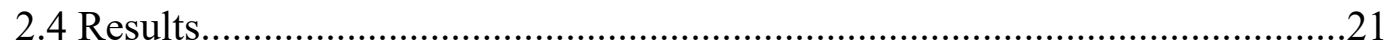

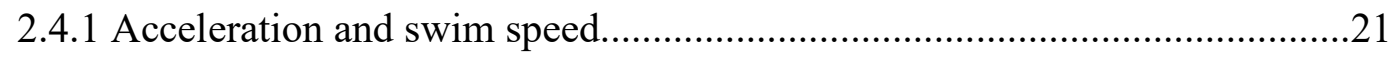

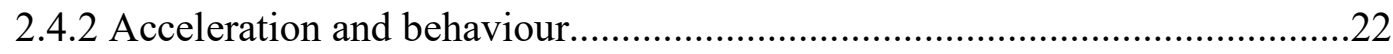

2.4.3 Bonefish behaviour in the wetland.....................................................22

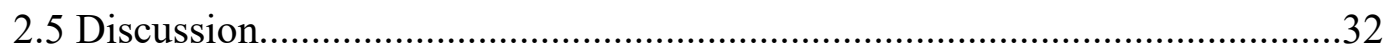

\section{Chapter 3. Spatiotemporal drivers of energy expenditure in a coastal marine}

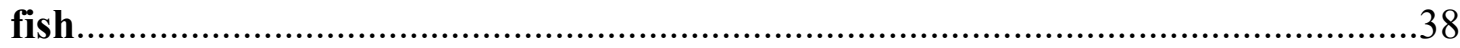

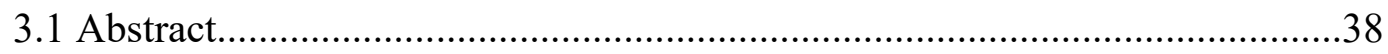

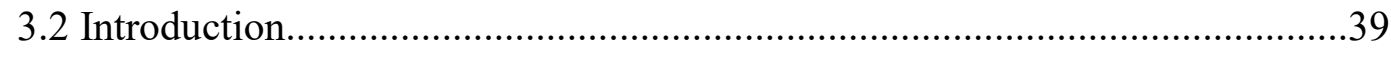

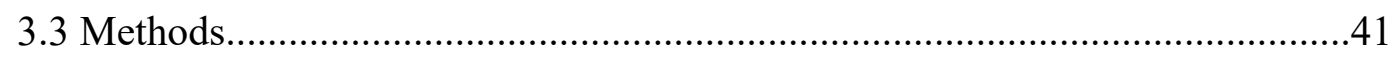

3.3.1 Accelerometer calibration................................................................4

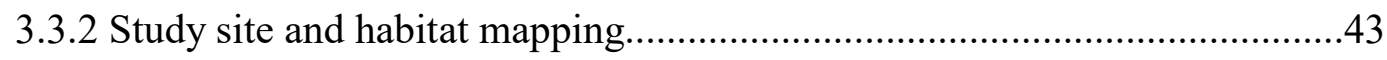

3.3.3 Acoustic tracking system and fish tagging.........................................45

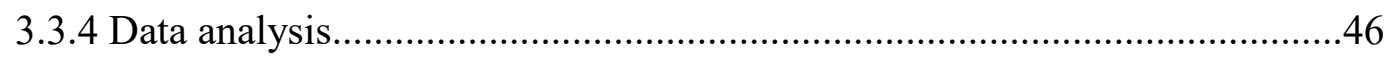

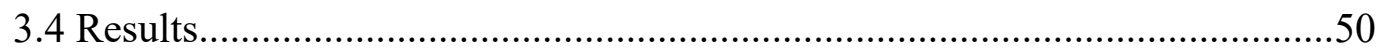

3.4.1 Accelerometer calibration.............................................................50

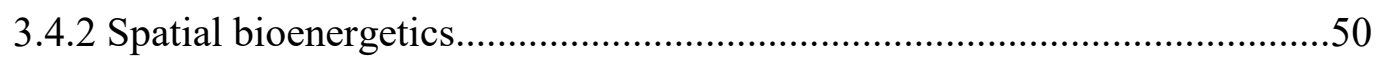

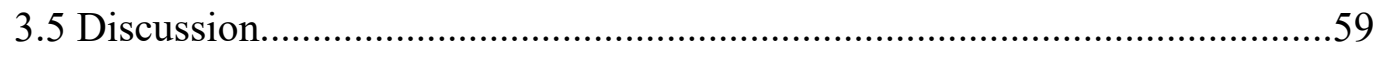

Chapter 4. Ecological energetics of a marine teleost fish across ecogeographic

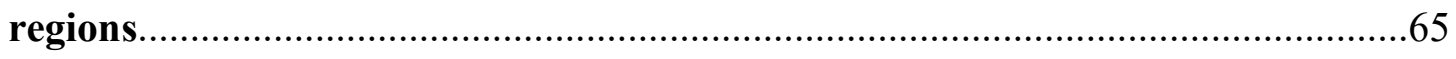

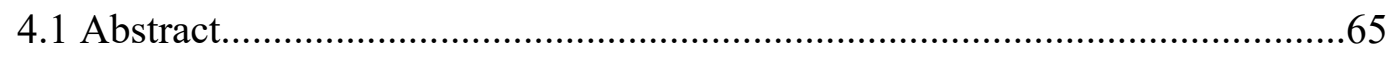

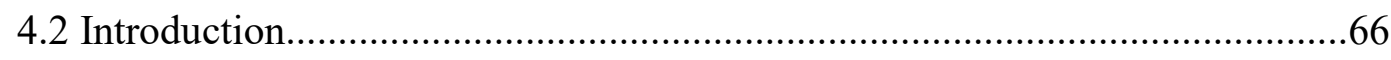

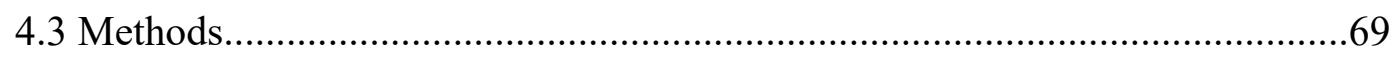

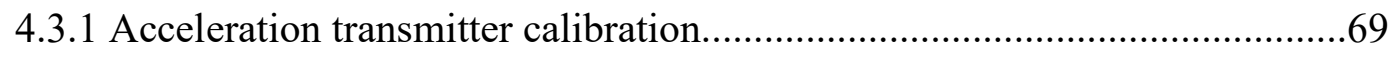


4.3.2 Field estimates of spatial energetics................................................ 70

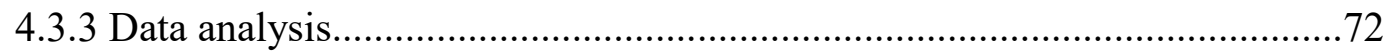

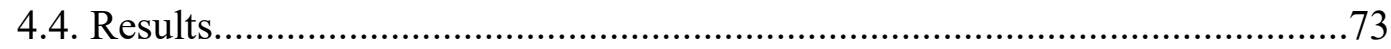

4.4.1 Activity, temperature, and energy expenditure...................................... 73

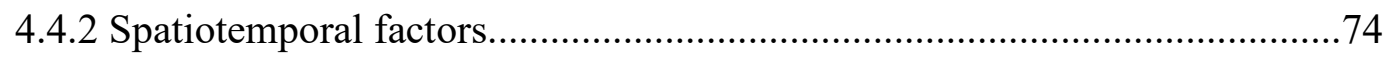

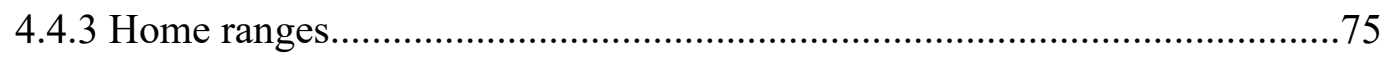

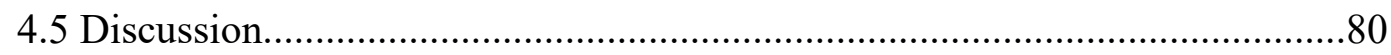

Chapter 5. Recovery bags reduce post-release impairments in locomotory activity and behaviour of bonefish following exposure to angling-related

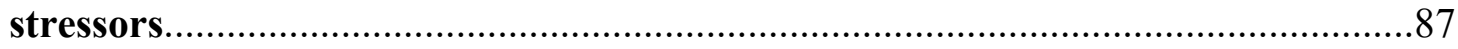

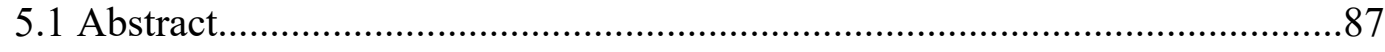

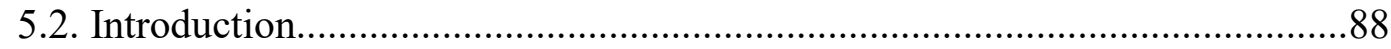

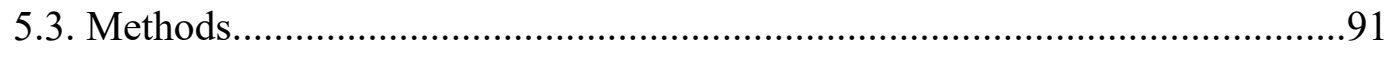

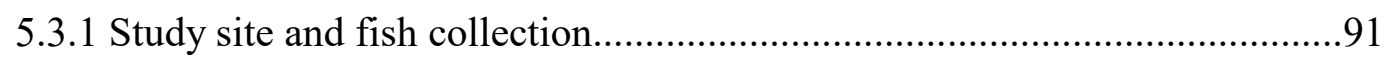

5.3.2 Validation of reflex impairment indices..........................................92

5.3.3 Post-release activity experiments......................................................93

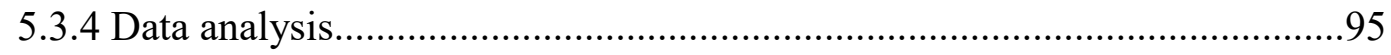

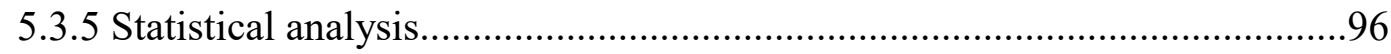

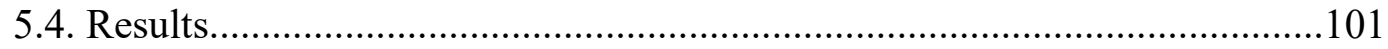

5.4.1 Validation of reflex impairment indices............................................101

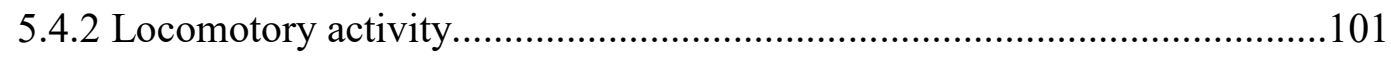

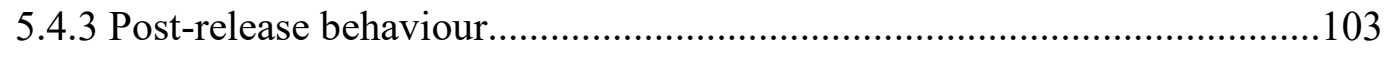

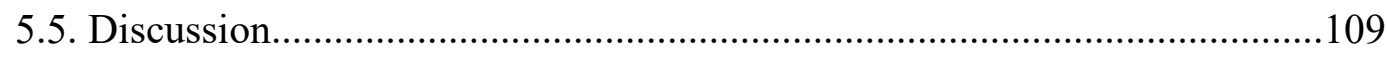

Chapter 6. Fishing-related stressors inhibit refuge-seeking behaviour in released

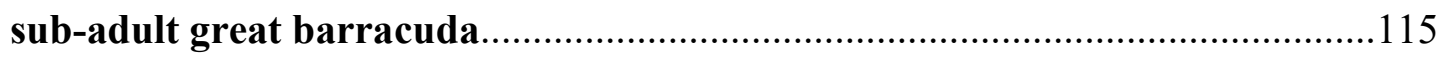




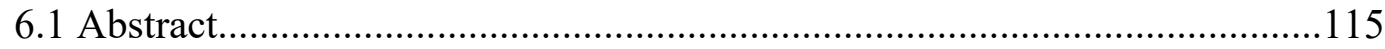

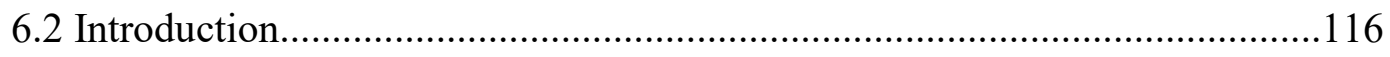

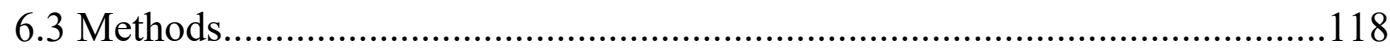

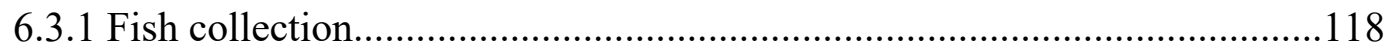

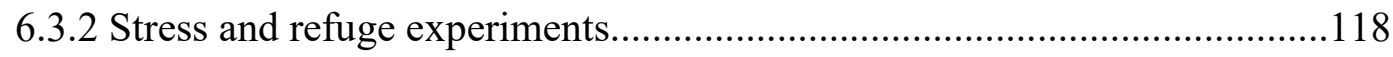

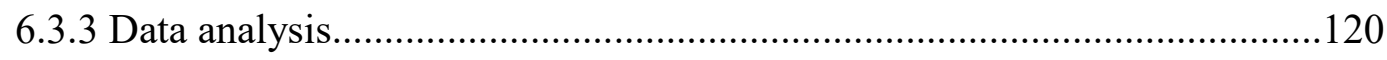

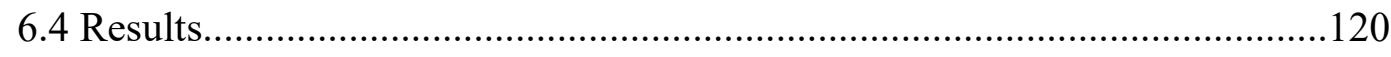

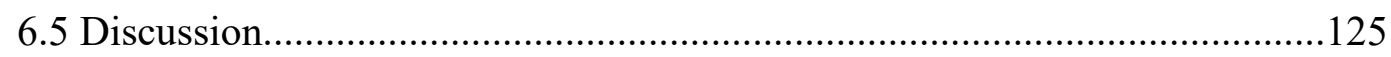

Chapter 7. Synthesis and future research directions....................................128

7.1 Environmental impacts on behaviour and energetics............................. 128

7.2 Anthropogenic impacts on behaviour.................................................... 140

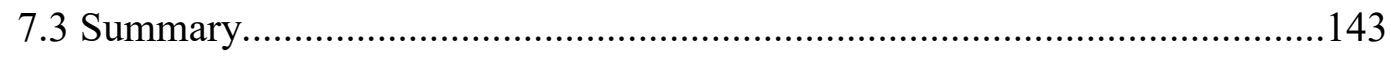

Appendix A. Abstracts of non-thesis publications during doctoral studies.........145

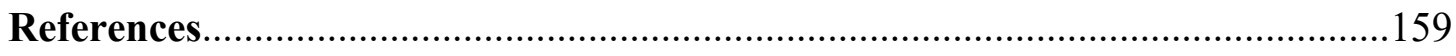




\section{List of Tables}

Table 2.1: Observed bonefish behaviours, corresponding accelerometrics, criteria, and identification efficiency using tri-axial accelerometer loggers. Under Criteria, Swim refers to time post-swimming, and $\mathrm{z}$ represents dynamic acceleration in the sway axis. All accelerometrics are presented as mean $\pm \mathrm{SD}$ (min-max)

Table 2.2: Significant factors predicting bonefish swimming behaviour (\% time), mean swimming speeds $(\mathrm{m} / \mathrm{s})$, and foraging behaviour (\# events/hr) in the wetland mesocosm over a 5-day period using generalized least squares models

Table 3.1: Linear mixed effects model outputs for bonefish acceleration (measured with a V13AP transmitter) and metabolism $\left(\mathrm{MO}_{2}\right)$ measured in a swim tunnel respirometer.

Table 3.2: Significant predictors of bonefish energy expenditure $\left(\mathrm{J} \mathrm{min}^{-1} \mathrm{~kg}^{-1}\right)$ derived from accelerometer transmitters within a fine scale positioning system on Las Pelas reef flat, comparing degrees of freedom (df), Aikaikes information criterion (AIC), log-ratio test (LRT), and p value based on the chi-square distribution (p-value) between full and reduced models .58

Table 4.1: Linear mixed effects model outputs for bonefish energy expenditure $\log ($ Joules min-1 kg-1) with both locations (Combined), predictors including location, month, bonefish fork length, and location*month interaction as predictors, as well as in each location separately (Culebra, Eleuthera) with habitat, diel period, and tide state as predictors. For all models fish ID was included as a random effect .79 


\section{List of Figures}

Fig. 2.1: Relationship between swimming speed (body lengths/second) and overall dynamic body acceleration (ODBA, g) and the fitted linear mixed model (black line) with upper and lower 95\% CI (hatch lines). Each symbol type represents individual fish ( $\mathrm{n}=9)$.

Fig. 2.2: Example of accelerometric signals from the dynamic sway (tail beat) axis and static surge axis (pitch) during observed bonefish behaviours while in the wetland excluding resting 25

Fig. 2.3: An untagged bonefish foraging in the wetland mesocosm. Photo credit: Karen Murchie.

Fig. 2.4: Individual bonefish swimming behaviour (\% time), mean swimming speed $(\mathrm{m} / \mathrm{s})$, and foraging (\# events/hr) across the diel period (dawn, day, dusk, night) in the wetland mesocosm.

Fig. 2.5: Individual bonefish swimming behaviour (\% time) across temperature and tidal periods while in the wetland mesocosm.

Fig. 3.1: Las Pelas fringing reef in Culebra, Puerto Rico. Colours represent habitat types identified using a k-means clustering algorithm based on habitat samples, and predicted across the entire area using a maximum likelihood classification algorithm on satellite imagery. Red circles represent acoustic receiver locations, and black crosses synchronization tags, comprising the Vemco Positioning System.

Fig. 3.2: A) Bonefish swimming speed $\left(\mathrm{m} \mathrm{s}^{-1}\right)$ and acceleration $\left(\mathrm{m} \mathrm{s}^{-2}\right)$ measured with V13AP transmitters in a swim tunnel and fitted linear mixed effects model predictions $\pm 95 \% \mathrm{CI}, \mathrm{B})$ Bonefish swimming speed $\left(\mathrm{m} \mathrm{s}^{-1}\right)$ and metabolic rate $\left(\mathrm{MO}_{2} ; \mathrm{mg} \mathrm{O}_{2} \mathrm{~min}^{-1} \mathrm{~kg}^{-1}\right)$ and linear mixed effect model predictions for a $61 \mathrm{~cm}$ fork length bonefish at $22^{\circ} \mathrm{C}$ (solid line), and a $34 \mathrm{~cm}$ fish at $36^{\circ} \mathrm{C}$ (hatched line) $\pm 95 \%$ CIs. 
Fig. 3.3: Linear mixed effects model predictions $\pm 95 \%$ CIs of bonefish energy expenditure ( $\mathrm{J} \mathrm{min}^{-1} \mathrm{~kg}^{-1}$ ) by A) bonefish fork length, B) habitat type and diel period, C) habitat type and tide state, D) habitat type and season

Fig. 3.4: Bonefish energy expenditure $\left(\mathrm{J} \mathrm{min}^{-1} \mathrm{~kg}^{-1}\right)$ on Las Pelas reef flat during each diel period.

Fig. 3.5: A) Bonefish acceleration $\left(\mathrm{m} \mathrm{s}^{-2}\right)$ and energy expenditure $\left(\mathrm{J} \mathrm{min}^{-1} \mathrm{~kg}^{-1}\right)$ across a range of water temperatures, B) Water temperature $\left({ }^{\circ} \mathrm{C}\right)$ and bonefish activity (acceleration, $\mathrm{m} \mathrm{s}^{-2}$ )

Fig. 3.6: Frequency of water temperatures in Las Pelas when tagged bonefish were present (white bars), and overall during the study period (grey bars).

Fig. 4.1: A) Bonefish activity (acceleration) across months of the year in Culebra (black) and Eleuthera (grey), B) associated environmental temperatures, C) predicted bonefish energy expenditure (Joules $\mathrm{min}^{-1} \mathrm{~kg}^{-1}$ ) from a linear mixed effects model

Fig. 4.2: Bonefish energy expenditure (Joules $\min ^{-1} \mathrm{~kg}^{-1}$ ) in Culebra (black) and Eleuthera (grey) across A) diel periods, B) tide states, C) habitats. Error bars represent $95 \%$ confidence intervals

Fig. 4.3: Home ranges (95\% minimum convex hulls; green) of bonefish with the median home range size in A) Eleuthera, and B) Culebra. Yellow circles represent acoustic receiver locations; maps are equivalent in scale

Fig. 5.1: Location of accelerometer attachment on bonefish for quantifying behaviour after simulated angling stress. .98

Fig. 5.2: Recovery bag being used to retain bonefish after angling by wading, behind a boat, and fastened to a mangrove 
Fig. 5.3: Example of behaviour clusters derived from continuous wavelet transformation and $k$-means cluster analysis, categorized into resting (hatched line), slow swimming (light grey), moderate swimming (grey), and fast swimming (dark grey) behaviours from one bonefish during 30 minutes post-release after simulated angling stress. 100

Fig. 5.4: (A) Reflex action mortality predictor impairment scores (mean $\pm \mathrm{SE}$ ), and (B) proportion of impairment for five impairment predictors in bonefish after $0 \mathrm{~min}$ $(n=30), 2 \min (n=20), 4 \min (n=5)$, and $6 \min (n=5)$ of air exposure $\pm S E$.

Fig. 5.5: Bonefish swimming activity (dynamic acceleration, g) in the sway axis measured using tri-axial accelerometers during the first minute post-release after exposure to simulated angling stress and (A) retained in a recovery bag for 15 minutes prior to release, or (B) immediately released. Oscillations represent individual tail beats. Data shown are from the individual bonefish that exhibited median activity levels for their respective treatments in the first minute postrelease.

Fig. 5.6: Mean and maximum tail beat frequencies and amplitudes of bonefish for 30 minutes post-release after exposure to simulated angling stress and retained in a recovery bag for 15 minutes prior to release (grey; $n=10$ ), or immediately released (white; $\mathrm{n}=9$ ) $\pm \mathrm{SE}$. * indicates $\mathrm{p}<0.05, * * \mathrm{p}<0.001$. Tail beat metrics were measured with tri-axial accelerometers and derived from continuous wavelet transformation. 106

Fig. 5.7: Dynamic acceleration (g) in the sway axis (tail beats) categorized as resting, slow, moderate, and fast swimming behaviours. Data shown is from one bonefish; collected within 30 minutes after angling-related stress.

Fig. 5.8: Frequency of bonefish behaviours (mean \pm SE) for 10 minutes post-release after exposure to simulated angling stress and retained in a recovery bag for 15 minutes prior to release (grey; $n=10$ ), or immediately released (white; $n=9$ ).........108 
Fig. 6.1: Great barracuda RAMP scores across three treatments prior to release into

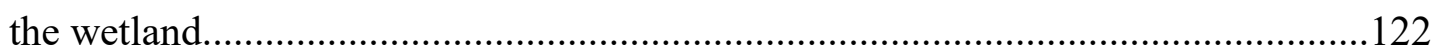

Fig. 6.2: Proportion of great barracuda in refuge with time post-release into the wetland across treatments $(\mathrm{Control}=$ solid, Chase $=$ dashed, Chase + air exposure $=$ dotted)

Fig. 6.3: (A) Time spent swimming (\%) by great barracuda, (B) Number of direction changes/min by barracuda across three treatments prior to reaching cover in the wetland. Dissimilar letters indicate significant differences. 124

\section{List of Appendices}

Appendix B Abstracts of non-thesis publications during doctoral studies. 


\section{Chapter 1. General introduction}

\subsection{The basics - Animal behaviour and energetics}

Animal behaviour is a central aspect of ecology. It is an integral component of individual fitness that can have cascading effects on population dynamics and demography (Shorrocks \& Swingland 1990; Morales et al. 2010). Behaviours such as movement, dispersal, and habitat selection also determine spatial distributions of animals (Lima \& Zollner 1996; Morales \& Ellner 2002; Nathan et al. 2008). Behaviour influences how individuals and populations interact with each other on both spatial and temporal scales, so it is pertinent to community and ecosystem dynamics (Naiman 1988; Morris 2003). Putatively, due to natural selection animals behave in ways that maximize fitness (Darwin 1859). Fitness is the outcome of much more than the reproductive process; animals must survive to maturity and acquire resources to fuel metabolic maintenance, activity, somatic growth, and gamete development. To this end, animals adopt a diversity of behaviours throughout their lifetime (Dugatkin 2004). The mechanisms driving animal behaviour are often considered within the framework of Nikolaas Tinbergen's four questions, which consider both the proximate (causation, ontogeny) and ultimate (adaptation and phylogeny) causes (Tinbergen 1963, 2005). In the age of the 'Anthropocene' (Crutzen 2006), with unprecedented human impacts on the environment, the intrinsic and extrinsic factors and their genetic and phenotypic bases have important implications not just in fundamental biology, but also in applied conservation (Caro 1999; Buchholz 2007). 
Animal physiology is also highly relevant to fundamental and applied ecology; the ability of animals to persist in an environment is related to their capacity to function within its ecological constraints (Withers 1992; Cooke et al. 2006). Energy is the currency of life; it is required by animals for metabolic maintenance, activity, growth, and reproduction (Kleiber 1961; Tytler \& Calow 1985; Brown et al. 2004). Energy gain and hence, fitness can be maximized through diverse behavioural tactics involving movement, habitat selection, foraging, predator avoidance, and reproduction (Cody 1974; Sih 1980; Pyke 1984; Pettifor et al. 1988). However, animals must make decisions on how much time and energy they commit to various behaviours necessary for fitness. For example, time and energy spent on predator avoidance often comes at the expense of foraging (Milinski \& Heller 1978; Hugie \& Dill 1994; Lind \& Cresswell 2005). Therefore individual fitness may vary due to environmental variables such as distribution of resources, costs of movement, intraspecific competition, and predation risk (Lima \& Dill 1990; Wilson et al. 1991; Lima \& Zollner 1996). Hence, living in heterogeneous environments, animals are thought to adopt a wide range of survival tactics. Measurements of animal behaviour and energy dynamics in relation to ecological characteristics help us understand population dynamics, distributions, and habitat use mechanistically (Nathan et al. 2008; Tomlinson et al. 2014; Chown \& Gaston 2016).

There is growing interest in understanding the mechanics behind how landscape level variation in ecological conditions influences animal distributions and trait diversity, with the recently emerging fields of macrophysiology (Chown \& Gaston 2016) and functional biogeography (Violle et al. 2014). Emphasizing energy as a key currency, the 
field of ecological energetics focuses on the influence of ecological factors on the energy dynamics of organisms; otherwise put, how ecological conditions impose physiological limitations on organisms (Bradshaw 2003; Tomlinson et al. 2014). A prominent constituent in this field is the metabolic theory of ecology (MTE; Brown et al. 2004), which posits that energy is a currency that can describe diverse ecological dynamics, scaling from individual to population, community, and entire ecosystem level processes. There is substantial evidence that patterns of animal metabolic rates across broad geographic space can be described primarily by animal size and environmental temperature (West et al. 1997; Gillooly et al. 2001; Brown et al. 2004). However, this is certainly a simplified representation of animal energetics, which can also be influenced by life stage, trophic level, habitat, activity, and primary productivity (Nagy 1987; Bryant 1997; Nagy et al. 1999). The MTE interfaces with the pace of life theory, which posits that animal life history characteristics (e.g., growth rate, maturation rate, life span) vary systematically with environmental temperature (Ricklefs \& Wikelski 2002; Wikelski et al. 2003). Originally developed focusing on endotherms, the pace of life tends to decrease (i.e., slower growth, longer life span) with increasing latitudes and corresponding environmental temperatures (Wikelski et al. 2003). However, in ectotherms the pattern is the opposite, with a faster pace of life at the equator (Angilletta et al. 2004). Indeed, temperature is considered the primary environmental driver of metabolic rate in ectotherms due to its influence on metabolic processes and locomotor performance (Clarke \& Johnson 1999; Halsey et al. 2015). 
While the MTE and pace of life theory provide the foundations for describing how animal characteristics (e.g., metabolic rate, growth rate) vary across ecogeographic regions, there are certainly discrepancies, likely due to variation in animal physiology and the impacts of diverse environmental factors (O’Connor et al. 2007; Irlich et al. 2009). The energetic costs of movement can vary dramatically across the landscape and between habitat types, forming the 'energy landscape' (Wilson et al. 2012; Shepard et al. 2013). For example, the energetic costs of movement can vary due to substrate type, water flow, or wind speed (Wilson et al. 1991; Sapir et al. 2011; McElroy et al. 2012). Energy landscapes explain animal movement ecology; for example, Wall et al. (2006) found African savannah elephants (Loxodonta africana) alter their movement paths to avoid energetically costly terrain. Similarly, birds use wind and fish use water flow patterns to minimize energetic costs of movement (Sapir et al. 2011; McElroy et al. 2012).

While quantifying the direct effects of landscape features on animal energetics provides a mechanistic basis for understanding movement ecology, there are many other environmental factors that impact animal energetics in the wild, and quantifying these impacts is particularly relevant to understanding spatiotemporal patterns of individualand population-level characteristics such as growth and reproduction. Prey availability and distribution are key factors in this context (Weiser \& Stamp 1998; Graeb et al. 2004). Predation risk can also have a significant impact on growth due to increased energy expenditure and reduced foraging opportunity (Werner et al. 1983; Halpin 2000; Biro et al. 2004). Lemon (1991) showed that the distribution of resources has a significant influence on net energy gain in zebra finches (Taeniopygia guttata), which in turn affects 
individual- and population-level growth rates. The field of ecological energetics describes how various environmental and ecological factors influence the energy dynamics of animals (Walter 1971; Stalmaster et al. 1984; Bell 1990), and is also useful for predicting the impacts of anthropogenic change on animal traits, distributions and persistence (Tomlinson et al. 2014).

\subsection{Applied conservation - Anthropogenic impacts}

Growing human populations have caused major declines in the biodiversity and abundance of organisms through habitat alteration, destruction, and overexploitation (Tillman et al. 1994; Pimm 1995; Rosser \& Mainka 2002). These stressors impact animal fitness directly through immediate mortality, or indirectly through physiological stress and altered behaviour (Pimm \& Raven 2000; Beale 2007), which consume energy and time that could be otherwise spent on fitness-enhancing activities such as feeding, growth, or reproduction (Frid \& Dill 2002). While animals adopt characteristics that maximize fitness in certain habitats, these strategies may not be optimal when their environment is drastically altered by human activity (Robinson et al. 1995). As human populations continue to grow and expand, so does our stress on the environment (Vitousek et al. 1997), and quantifying effects of anthropogenic disturbances on animal fitness is becoming increasingly important.

Animal behaviour has an important role to play in conservation biology (Soulé 1985; Caro 2007). The impact of anthropogenic stressors on animal fitness, and even 
ecosystem dynamics is mediated by animal behaviour. As an example, quantification of animal movement patterns is essential for designing protected areas (Kramer \& Chapman 1999), while social structures can influence responses to exploitation (Milner-Gulland et al. 2003). However, behaviour is often neglected in the context of conservation (see Angeloni et al. 2008). This is particularly true for basic behavioural biology (Buchholz 2007), yet understanding the ultimate causes of animal behaviour is of particular interest for conservation of animals in environments that are drastically altered by humans (Schlaepfer et al. 2002). Interfacing behaviour with elements of energetics or other aspects of ecological and environmental physiology is a particularly powerful approach for understanding how human activities will influence organisms, populations and ecosystems (Cooke et al. 2014a).

While wildlife exploitation by humans is traditionally consumptive (Robinson \& Redford 1991), non-consumptive exploitation (e.g. wildlife observation, catch-andrelease fishing) is increasing in popularity, particularly in developed countries where people rely less on wildlife resources for sustenance (Walpole \& Thouless 2005). Nonconsumptive exploitation is often touted as a method to generate revenue and engender human interest in conservation for the resource. However, it can also have negative impacts on wildlife populations, inadvertently causing physiological stress and altering behaviour (Green \& Higginbottom 2000; Blanc et al. 2006). While consumptive exploitation impacts populations directly by removing individuals, the impacts of nonconsumptive exploitation are often more subtle, with sublethal physiological effects that 
accumulate into reduced fitness over time, and in some cases eventual changes in space use (Lusseau \& Bejder 2007).

\subsection{Fish as model species}

Fish represent an important resource for humans for recreation and consumption (Cooke \& Cowx 2006). Fish are also integral to aquatic ecosystem function, and hence, relevant to not only demand-derived, but also fundamental ecosystem services (Holmund \& Hammer 1999). However, fish are the most over-exploited group of animals on Earth, and have undergone massive declines in abundance and biodiversity (Hutchings et al. 2004; Pauly et al. 2005). Fish also occupy nearly every type of aquatic environment, exhibiting massive diversity in taxonomy, morphology, physiology, and behaviour (Godin 1997; Helfman et al. 2009). Therefore fish offer excellent model organisms to inform basic behavioural ecology and applied management. Similarly, coastal marine ecosystems offer diverse and abundant resources for humans, yet are some of the most heavily affected by human activities (Vitousek et al. 1997; Castilla 1998; Halpern et al. 2008).

Bonefish (Albula spp.) are a group of at least 12 species that occupy shallow tropical and sub-tropical seas worldwide (Alexander 1961; Wallace 2014). Two species are prevalent in the Caribbean and Atlantic Seas, Albula vulpes and Albula species B (Wallace 2014). However, Albula vulpes is the only species found in shallow nearshore habitats in both Culebra and Eleuthera (Danylchuk et al. 2007; Brownscombe et al. 2015 
and are the main focus of this thesis. Bonefish occupy a wide range of habitats, including sand, mud, and marl flats, coral reefs, and sea grass beds (Colton \& Alevizon 1983a,b; Adams et al. 2012). Bonefish move into shallow areas in intertidal zones with flooding tides (Colton \& Alevizon 1983a; Humston et al. 2005, Murchie et al. 2013) to feed on a range of benthic prey including primarily crabs, shrimp, molluscs, worms, and small fish (Colton \& Alevizon 1983b; Crabtree et al. 1998), returning to deeper water habitat with ebbing tides. Because of this diurnal feeding migration, bonefish are thought to play an important role in the movement of nutrients offshore in shallow seas (Ault 2008; Murchie et al. 2013), although this has not been well quantified to date. Bonefish exhibit some significant variability in life history characteristics, growing faster in Puerto Rico and Florida than in The Bahamas (Crabtree et al. 1996, 1997; Adams et al. 2008), although there is currently limited information on life history characteristics and population dynamics in most areas of their range. Genetics studies revealed a high level of connectivity between the diverse regions bonefish occupy in The Caribbean and Western Atlantic Seas (Wallace 2014) likely due to their reproductive strategy involving offshore broadcast spawning (Danylchuk et al. 2011), and an extended pelagic leptocephalus larval stage (Pfeiler et al. 1988).

Bonefish are not only ecologically important, but also generate significant revenue for local economies due to their popularity for recreational anglers (Humston 2001; Ault 2008; Danylchuk et al. 2008). While bonefish angling is almost entirely catchand-release, post-release predation rates can be very high (e.g., 40\%; Cooke \& Philipp 2004) due to behavioural impairment and high densities of opportunistic predators 
(Cooke \& Philipp 2004; Danylchuk et al. 2007a,b), raising concerns over the sustainability of this practice for bonefish populations. Further, many coastline habitats are being altered in many areas of their range (Lotze et al. 2006) and commercial gill nets are another major source of bonefish mortality (Danylchuk et al. 2008). Unfortunately, $A$. vulpes was recently designated Near Threatened on the IUCN Red List, citing declines in the Florida Keys, St. Croix, Bermuda, and the Yucatan Peninsula, with an estimated 20\% decline in bonefish habitat in the past 35 years (Larkin et al. 2011; Adams et al. 2012).

Great barracuda (Sphyraena barracuda) are a common predatory fish in coastal areas of tropical and sub-tropical seas worldwide (de Sylva 1963). As juveniles, great barracuda occupy shallow water habitats, including mangrove creeks, sand and seagrass flats (Paterson 1998). Juveniles adopt piscivory at a very young age, and as they grow into adults, great barracuda move into deeper waters ranging from 1 to $>50$ meters, but still tend to associate with structured habitats such as reefs and flats where they forage opportunistically on diverse fish species (de Sylva 1963). Adults grow very large, in excess of 2 meters, and are considered a top predator in many marine systems. Because of their role as a ubiquitous predator in shallow tropical marine ecosystems, great barracuda are an important component of the food web (Friedlander \& DeMartini 2002). Great barracuda are a sport fish, commonly targeted by recreational anglers and caught incidentally as by-catch as adults and sub-adults (O'Toole et al. 2010). In areas of high fishing pressure such as Florida, population declines have been occurring (FWC 2015). However, release rates are often high with this species due to their tendency to 
biomagnify ciguatera bacteria, which produce a toxin with negative effects on human health when consumed (Villareal et al. 2007; O’Toole et al. 2010).

\subsection{Recreational fisheries}

Recreational fishing is a highly popular activity worldwide and an important connection between humans and natural resources with deep cultural roots in both sustenance and recreation (Cooke \& Cowx 2004; Arlinghaus \& Cooke 2009). While traditionally harvest dominated, catch-and-release (C\&R) fishing via angling with rod and reel is increasing in popularity due to harvest regulations and conservation priorities of anglers (Cowx 2002;

FAO 2012; Freire et al. 2012; Brownscombe et al. 2014). With growing human populations, release of captured fish is essential for sustainable fisheries and fish conservation. As a conservation strategy, $\mathrm{C} \& \mathrm{R}$ relies on the assumption that released fish will survive and experience limited fitness consequences (Wydoski 1977; Arlinghaus et al. 2007). However, angling often elicits physical injuries due to hooking, landing, and handling, as well as physiological stress due to exhaustive physical exercise while the fish resist capture, and subsequent air exposure during unhooking and admiration by the angler (reviewed by Cooke \& Suski 2005; Pelletier et al. 2007; Brownscombe et al. 2016a). For these reasons, released fish can experience delayed mortality or reduced fitness. Under high exploitation rates, through a combination of harvest and the sublethal impacts of C\&R, recreational fisheries can have significant detrimental impacts on fish populations and their ecosystems (Cooke \& Cowx 2004; Lewin et al. 2006). It is 
therefore essential to develop angling practices and regulations that minimize impacts on exploited fishes to ensure the sustainability of this culturally important connection to wildlife resources and conserve fish populations.

As with all human-wildlife interactions, animal behaviour is an important mediator of the effects of $\mathrm{C} \& \mathrm{R}$ on fish fitness. For example, in many fisheries, postrelease behavioural impairment results in increased predation risk by opportunistic predators, which can be significant source of mortality in many types of fisheries around the world (Danylchuk et al. 2007a; Raby et al. 2014). In ecosystems with high predator burden (e.g., shallow tropical marine systems), some fish species experience post-release predation rates exceeding 40\% (Cooke \& Philipp 2004; reviewed in Raby et al. 2014). Studies on the topic have identified locomotory impairment and the release of chemical cues such as ammonia that attract predators as causal factors of increased post-release predation risk (Danylchuk et al. 2007a; Dallas et al. 2010; Cooke et al. 2014b). However, studies explicitly quantifying behavioural impairment and related causal factors in this context are scarce. Understanding the behavioural mechanisms leading to increased predation risk in recreational fisheries would help contribute to more sustainable fisheries, as well as greater understanding of the effects of anthropogenic stressors on wildlife welfare in general. 


\subsection{Hypothesis and objectives}

At this time of unprecedented environmental change due to rapidly increasing anthropogenic activities, studies of fundamental animal ecology as well as applied conservation are essential for managing and conserving natural resources and resolving growing human wildlife conflicts. For this reason, my Ph.D. dissertation explores animal behaviour and energetics at the interface of basic and applied contexts. My overarching hypothesis is that ecological and anthropogenic factors influence animal behaviour and energetics. I test this hypothesis in five studies focusing on two fish species. There is a logical flow, particularly from Chapters 2-4, which examine the ecological drivers of behaviour and energy dynamics, and in Chapters 5-6 focusing on the impacts of anthropogenic stressors on fish behaviour in the context of post-release predation risk. In Chapter 2 I explore the hypothesis that both intrinsic and extrinsic factors influence bonefish behaviour, including activity levels and foraging. After establishing a basic understanding of bonefish behaviour and ecological drivers, I then examine the hypothesis that landscape features and temporal factors influence bonefish behaviour and energetics in the wild from fine (Chapter 3) to broad (Chapter 4) spatial scales, providing a comprehensive assessment of the influence of ecological factors, with implications for behavioural ecology and population dynamics. In Chapter 5 I explore the hypothesis that recreational angling influences bonefish behaviour and locomotory capabilities, causing increased post-release predation risk. Based on my findings in Chapter 5, in Chapter 6 I explore the hypothesis that cognitive impairment is a causal factor in post-release predation risk using great barracuda as a model. 


\section{Chapter 2. Foraging behaviour and activity of a marine benthivorous fish estimated using tri-axial accelerometer biologgers}

\subsection{Abstract}

Fine-scale behaviour such as foraging is difficult to quantify in free-swimming wild fish, yet has important basic and applied implications. Here, I used tri-axial accelerometer biologgers to determine accelerometric predictors of bonefish (Albula vulpes) behaviours (resting, swimming, bursting, coasting, and foraging) in a wetland mesocosm in Eleuthera, The Bahamas. I also used a swim flume to estimate the relationship between acceleration and swimming speed for bonefish $(n=9)$. In the wetland study, five bonefish were tagged externally with accelerometer loggers and monitored for a 5-day period during which visual behavioural observations were conducted for 5 hours. Classification tree models were used to identify accelerometric criteria for bonefish behaviours, and a classification algorithm was applied to estimate behavioural frequencies for bonefish in the wetland for the 5-day period. Bonefish spent the majority of time resting (57\%), followed by swimming (26\%) and coasting (17\%), and foraged an average of $11 \mathrm{times} / \mathrm{hr}$. Bonefish exhibited primarily slow swimming speeds (average $0.18 \mathrm{~m} / \mathrm{sec}$ ) while in the wetland, with occasional burst swimming events (14/hr) to maximum swimming speeds ranging from 4.3-6.4 m/sec across individuals. Swimming and foraging behaviours varied among individuals and over the diel cycle. Bonefish generally exhibited the most swimming behaviour at dawn, and the most foraging behaviour at night. Temperature and tide were also significant predictors of swimming behaviour, and fish were generally 
most active at lower temperatures $\left(\sim 24^{\circ} \mathrm{C}\right)$ and during ebbing tidal periods despite the fact that the wetland was disconnected from the ocean. The ability to estimate fine-scale behaviours such as foraging and activity levels has important implications for understanding energy dynamics, which is fundamental to the fitness of wild fish.

\subsection{Introduction}

Environmental and ecological factors influence the behaviour and energy dynamics of animals, with direct implications for their fitness (Lima \& Zollner 1996; Lind \& Cresswell 2005). For example, energetic costs of movement may vary among habitat types (Nathan et al. 2008; Shepard et al. 2013), while the distribution of resources can affect foraging success and behaviour (Pyke 1977). Energy is the currency of life for wild animals, and thus, energy dynamics have the potential to influence its allocation to different activities such as growth and gonadal development, influencing organismal fitness (Kleiber 1975; Callow 1985). Indeed, these energy dynamics can have population level effects as has been documented for a variety of taxa (e.g., Tytler \& Callow 1985; Lemon 1993; Nagy et al. 1999). While understanding how environmental conditions and animal behaviour interact is key for both basic biology (i.e. foraging theory; Altmann \& Altmann 2003) and applied conservation (Buchholz 2007), measurement of these dynamics in situ is challenging. A variety of biologging and biotelemetry tools with various sensors have been developed and they show much promise for the study of 
detailed aspects of animal behavior and energetics in natural environments, including aquatic ecosystems (Cooke et al. 2004; Rutz \& Hays 2009).

Tri-axial accelerometer loggers have been used to obtain estimates of animal behaviour and energy use in a wide range of species, from invertebrates to sharks (Halsey et al. 2011; Whitney et al. 2010; Robson et al. 2012; Brown et al. 2013) and even have potential to examine very fine-scale behaviour, such as foraging (Suzuki et al. 2009). To date, measurements of foraging behaviour have been restricted mainly to large species such as seals (Viviant et al. 2010) and sea turtles (Narazaki et al. 2013), although recent studies have used accelerometry to identify foraging behaviour of Atlantic salmon (Salmo Salar L.) in aquaculture cages (Føre et al. 2011), and feeding strikes by great sculpin (Myoxocephalus polyacanthoceaphalus) in laboratory tanks (Broell et al. 2013). While accelerometers show promise for measuring foraging behaviour in teleost fishes, to my knowledge, this technique has yet to be applied on wild fish in more natural environments.

Bonefish (Albula spp.) are a group of at least 12 fish species that occupy shallow tropical and sub-tropical seas worldwide (Alexander 1961; Wallace 2014), including a wide range of habitats; sand flats, coral reefs, and sea grass beds (Colton \& Alvezon 1983a,b; Cooke \& Philipp 2004; Danylchuk et al. 2007a). Bonefish move into shallow areas with flooding tides to feed, and return to adjacent deeper-water habitat with ebbing tides (Colton \& Alevizon 1983a; Humston et al. 2005; Murchie et al. 2013). Because of this feeding migration, bonefish are thought to play an important role in the movement of 
nutrients offshore in shallow seas (Murchie et al. 2013), including when some species form large aggregations and move offshore to spawn (Danylchuk et al. 2011). Bonefish are not only ecologically important, but also generate significant revenue for local economies through recreational angling (Humston 2001; Danylchuk et al. 2008). Therefore bonefish offer an excellent model for understanding behavioural ecology and energy dynamics to contribute to both basic biology and applied conservation.

Bonefish behaviour has been quantified using accelerometer transmitters (see Murchie et al. 2011a), however, this technology does not enable fine scale measurement of animal movement and posture required for certain behaviours (i.e., foraging). My objective was to examine fine-scale behaviour (e.g., swimming, resting, foraging) and swimming speeds of bonefish using tri-axial accelerometer loggers. To this end, I determined accelerometric predictors of these behaviours in an experimental wetland mesocosm, and swimming speeds a swim tunnel. I then estimated bonefish behaviours and swim speeds in the mesocosm over a 5-day period. In doing so, I aimed to develop methods for examining fine-scale behaviour and energetics, and gain insight into the behavioural ecology of bonefish. 


\subsection{Methods}

\subsubsection{Fish capture}

Bonefish used in both swim tunnel and wetland experimentation were captured by seine net in Kemps Creek $\left(24^{\circ} 48.9^{\prime \prime} \mathrm{N}, 76^{\circ} 18.1^{\prime \prime} \mathrm{W}\right)$ and held in a circular tank (3.7 m diameter $\mathrm{x} 1.25 \mathrm{~m}$ height; $13180 \mathrm{~L}$ ) for up to 1 week prior to experimentation. Tanks were supplied with constant flow (approximately 1,800 L/h) of fresh seawater and fish were fed daily rations of cut fish.

\subsubsection{Swim tunnel}

Nine bonefish $(41.3 \pm 2.9 \mathrm{~cm}$ FL; $897 \pm 162 \mathrm{~g})$ were tagged with tri-axial accelerometer loggers (model X8M-3, $500 \mathrm{mAh}$ battery, $15 \mathrm{~g}$ in air, $25 \mathrm{~Hz}$ recording frequency; Gulf Coast Data Concepts, Waveland, MS) externally through the dorsal musculature below the dorsal fin. Accelerometers were secured using plastic frontal and backing plates with $36 \mathrm{~kg}$ strength braided Dacron line. Once accelerometers were secured, bonefish were placed in a Blazka-style recirculating swim tunnel (24.1 internal diameter x $116 \mathrm{~cm}$ length) capable of generating laminar flows up to $2.1 \mathrm{~m} / \mathrm{s}$ (see Thorstad et al. 1997 for additional details on the swim tunnel). Fish were tagged the night prior to their trial and held in the swim tunnel overnight at a water velocity of $1 \mathrm{~cm} / \mathrm{s}$. This velocity allowed for circulation of fresh seawater, but was slow enough to allow the fish to escape the flow and rest on the bottom of the tunnel. Swimming trials were conducted between 0700 and 
1000h from January 18-27, 2013. Water velocity was increased in increments of 15 $\mathrm{cm} / \mathrm{sec}$ and accelerometrics were derived from a 1 min period where fish were visually observed as stationary (moving the same speed as the flow) in the tunnel. The observer's watch was time-synced with the biologgers upon launching the devices, and time drift was quantified upon reconnecting the devices, but was negligible $(<2 \mathrm{sec})$, ensuring accurate synchronization between observations and swimming speeds. Water velocity was increased until the fish could no longer maintain position in the tunnel.

\subsubsection{Wetland mesocosm}

Five bonefish $(45.6 \pm 2.7 \mathrm{~cm} \mathrm{FL} ; 1141 \pm 173 \mathrm{~g})$ were equipped with accelerometer loggers in the same manner as the swim tunnel study, and were released immediately after tagging into an $\sim 2500 \mathrm{~m}^{2}$ enclosed wetland mesocosm in Eleuthera on February 22, 2012. The wetland is situated adjacent to the sea, and receives a constant influx of seawater from a wetlab facility. It is vegetated with red mangrove (Rhizophora mangle), black mangrove (Avicennia germinans), and Halimeda (Halimeda spp.). Tagged bonefish remained in the wetland for 5 days, during which time behavioural observations were conducted by an observer from a raised bridge that runs through the wetland for 2 hours in the morning hours (0700-0900), 1 hour in the afternoon (1200-1400) and 2 hours in the evening (1630-1830). The observer noted the timing of behaviours including swimming, burst swimming, coasting, resting, and foraging using the same watch-logger synchronization as the swim tunnel study. Individual bonefish were identified by unique 
colouration of each accelerometer. A temperature logger (Thermochron iButton ver. DS1921G, -40 to $80^{\circ} \mathrm{C}$ range, Maxim CA) was placed in the center of the wetland for the course of the study.

\subsubsection{Data analysis}

Tri-axial accelerometer loggers were programmed to continuously record total acceleration (g) at $25 \mathrm{~Hz}$ frequency in three ( $\mathrm{x}=$ heave, $\mathrm{y}=$ surge and $\mathrm{z}=$ sway) axes, where total acceleration was the sum of both static (gravity) and dynamic (animal movement) acceleration with maximum values of $\pm 8 \mathrm{~g}$. Device output was calibrated by rotating the

device through known angles to real $\mathrm{g}\left(9.8 \mathrm{~m} \mathrm{~s}^{-2}\right)$ prior to deployment (as per Gleiss et al. 2010). Static and dynamic acceleration were separated by weighted smoothing at an interval of $2 \mathrm{sec}$. Overall dynamic body acceleration (ODBA) was calculated as the absolute sum of the dynamic acceleration from all three axes (see Gleiss et al. 2011). Spectral analysis was used to estimate frequencies and amplitudes in the sway axis, which comprised the dominant signal, and while swimming, indicates tail beat frequency. Spectral data were band-pass filtered to include frequencies of 0.5 to $10 \mathrm{~Hz}$ in the analysis. Pitch $\left({ }^{\circ}\right)$ was calculated from the static values of the surge axis $(\mathrm{g})$ using equation (1) below, where $\mu$ is the mean static value in the surge axis during a 1-minute period where each bonefish was observed to be resting level to the substrate. All analysis of accelerometer data was conducted using Igor Pro 6.0 software (WaveMetrics Inc., Lake Oswego, OR) and Ethographer (see Sakamoto et al. 2009). 
(1) Pitch $=\operatorname{arcsine}(\text { surge static- } \mu)^{*} 180 / \pi$

For bonefish in the swim tunnel, accelerometer-derived overall dynamic body acceleration (ODBA, g) was estimated using a linear mixed effect model with swimming speed $(\mathrm{BL} / \mathrm{s})$ as a predictor and individual fish as a random factor. Swimming speeds were corrected for blocking effect prior to analysis (Jones et al. 1974). Marginal and conditional $\mathrm{R}^{2}$ values were calculated as outlined in Nakagawa et al. (2013).

Using acceleration data from visually observed bonefish behaviours, classification trees (Breiman et al. 1984) were applied to determine accelerometric criteria to identify bonefish behaviours. Firstly, swimming behaviours (routine and burst swimming) were distinguished from non-swimming behaviours (resting, coasting) using ODBA (g), sway amplitude $(\mathrm{g})$, sway frequency $(\mathrm{Hz})$, pitch $\left(^{\circ}\right)$, and $\Delta$ pitch $\left(^{\circ}\right)$ as predictors. Time postswimming was used as additional predictor variable to classify coasting and foraging behaviours. A classification criteria algorithm was then used to identify bonefish behaviours for all five fish for the 5-day period they inhabited the wetland. During periods identified as swimming behaviour, swimming speeds were also predicted using the linear mixed effects model developed from the swim tunnel data.

Mesocosm data for bonefish swimming behaviour (\% time spent actively swimming), foraging behaviour (foraging events/hr), and average swimming speed (m/s) were analyzed with generalized least squares regression using a backwards modelselection procedure and log-ratio tests. The full models included the predictors: time of day (dawn, day, dusk, night), tide (low, flooding, high, and ebbing), study hour 
(continuous from the first hour of the study), temperature (continuous covariate), fish ID (categorical). To determine how individuals responded to release in the wetland and any changes in behaviour, two-way interactions included fish ID x Time of day, fish ID x temperature, fish ID x Tide, and fish ID x hour. Single-term deletions resulted in models that only contained significant terms at $\alpha=0.05$. A variance structure was used to correct for heterogeneity in the categorical factors (Pinheiro \& Bates 2000). Temporal autocorrelation was accounted for by including a correlation structure in each model (Pinheiro \& Bates 2000; Zuur et al. 2009). I validated each model by following the protocol of Zuur et al. (2009). Due to patterns in the residuals, foraging behaviour and average swimming speed were $\log _{10}$ transformed. All statistical analyses were conducted using RStudio (version 0.97.314) and R (version 2.15.3; R Core Development Team, 2012).

\subsection{Results}

\subsubsection{Acceleration and swim speed}

Accelerometer-equipped bonefish in the swim tunnel maintained swimming speeds from 0 to $2.1 \mathrm{BL} / \mathrm{s}$. A linear mixed model predicted ODBA from swimming speed (Fig. 1; Marginal $\mathrm{R}^{2}=0.82$, Conditional $\mathrm{R}^{2}=0.92$ ), which was defined by equation ( 2 ):

$\mathrm{ODBA}=0.412 \cdot \mathrm{BL} / \mathrm{sec}-0.012$ 


\subsubsection{Acceleration and behaviour}

There were minimum of seven instances of each type of behaviour visually observed for bonefish in the wetland (Table 2.1). Swimming behaviour consisted of high values of ODBA, sway frequencies and amplitudes (Table 2.1; Fig. 2.2). Swimming behaviour was classified by sway amplitudes $\geq 0.03 \mathrm{~g}$, while coasting behaviour was classified by $\mathrm{z} \geq 0.01 \mathrm{~g}, \leq 5 \mathrm{sec}$ post-swimming (Table 2.1 ). Otherwise sway amplitudes $<0.03 \mathrm{~g}$ were classified as resting (Table 2.1). Bonefish were considered to be burst swimming when sway amplitudes were $\geq 0.25 \mathrm{~g}$. Foraging behaviour was classified using three defining characteristics. The foraging event itself was classified by a sharp decline in pitch (minimum $-3^{\circ} / \mathrm{sec}$ ) to minimum pitch values of $-4^{\circ}$ (Table 2.1 ), consistent with the manner in which bonefish feed (i.e., head down and tail up; Fig. 2.3). Additionally, swimming behaviour always preceded ( $<=4 \mathrm{sec}$ prior) foraging behaviour, which was incorporated into the classification criteria. Using these criteria, an average of $97 \%$ of all visually observed behaviours were classified correctly; only $3 \%$ were misclassified.

\subsubsection{Bonefish behaviour in the wetland}

During the 5-day period that bonefish inhabited the wetland, they spent the majority of their time resting (57\%), followed by swimming (26\%) and coasting (17\%); foraging occurred on average 11 times/hr (Fig. 2.4). While swimming, bonefish maintained primarily slow speeds (mean $0.18 \mathrm{~m} / \mathrm{sec}$; Fig. 2.4) with occasional burst swimming 
activity (mean 14/hr) at maximum swimming speeds ranging from $4.2-6.4 \mathrm{~m} / \mathrm{sec}$ across individuals (Table 2.1).

Individual fish and diel period were significant predictors of bonefish swimming behaviour, swimming speeds, and foraging behaviour (Table 2.2; Fig. 2.4). The majority of fish were most active at dawn, but exhibited the highest mean swimming speeds during the day and at dusk (Fig. 2.4). Bonefish were also active at night, which is generally when most foraging behaviour occurred. Fishes 44 and 45 (cm FL) exhibited contrasting behavioural patterns. Fish 45 spent the least time swimming of all tagged fish, but exhibited the highest mean swimming speeds (Fig. 2.4). In contrast, Fish 44 exhibited the highest proportion of time swimming, with low average swimming speeds, and was also highly active at night, both swimming and foraging.

Environmental factors, temperature and tide were also significant predictors of bonefish swimming behaviour (Table 2.2). Bonefish were most active at low temperatures $\left(22\right.$ to $\left.25^{\circ} \mathrm{C}\right)$, and generally became less active as temperatures increased (Fig. 2.5). However, while fishes 42 and 45 generally followed this pattern, activity peaked again at the highest temperatures observed in the wetland $\left(26\right.$ to $\left.30^{\circ} \mathrm{C}\right)$. Across the tidal cycle, bonefish were generally most active during ebbing and low tidal periods, and least active during flooding tides (Fig. 2.5). 
Fig. 2.1: Relationship between swimming speed (body lengths/second) and overall dynamic body acceleration (ODBA, g) and the fitted linear mixed model (black line) with upper and lower 95\% CI (hatch lines). Each symbol type represents individual fish ( $\mathrm{n}=9$ ).

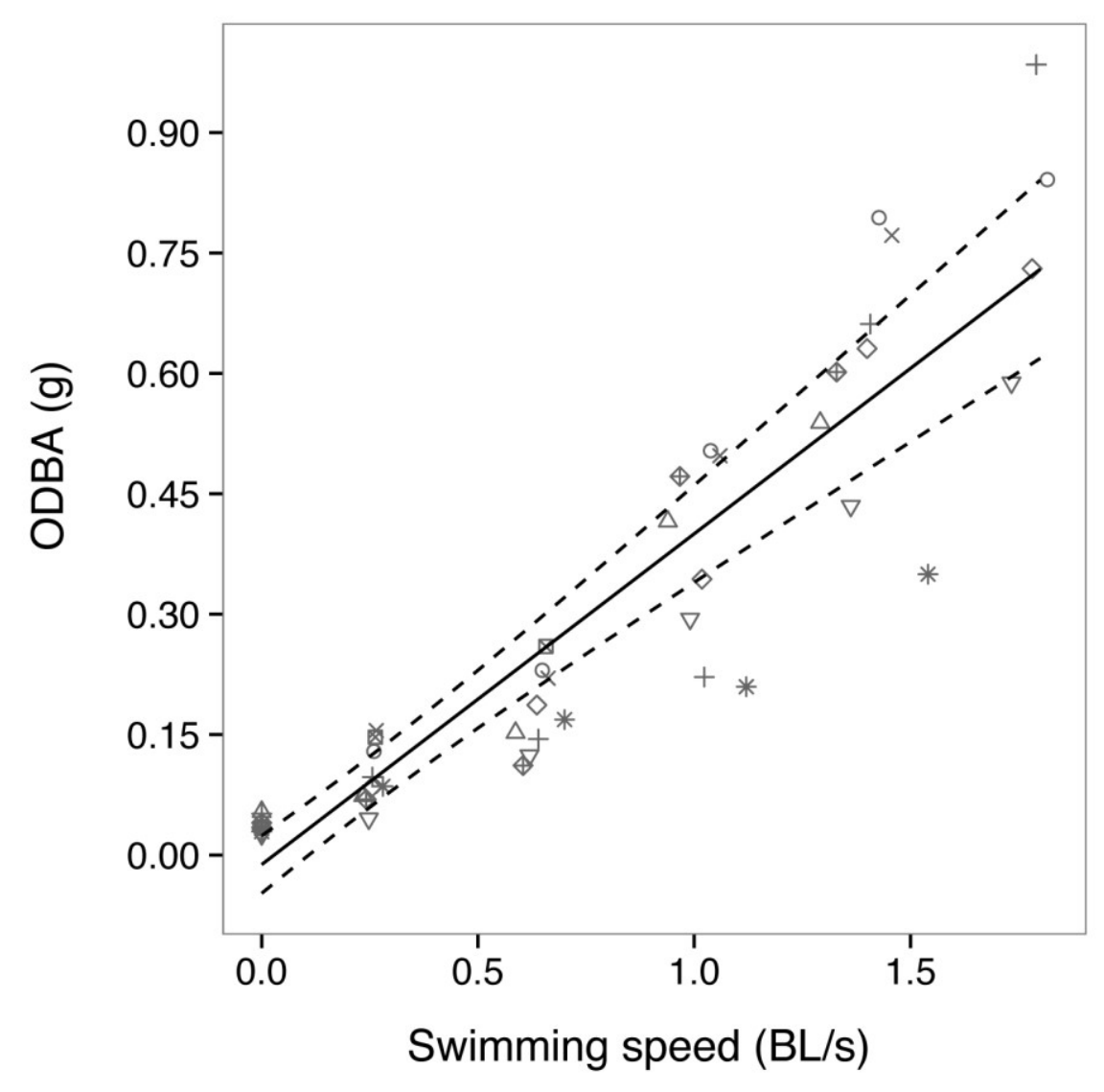


Fig. 2.2: Example of accelerometric signals from the dynamic sway (tail beat) axis and static surge axis (pitch) during observed bonefish behaviours while in the wetland excluding resting.

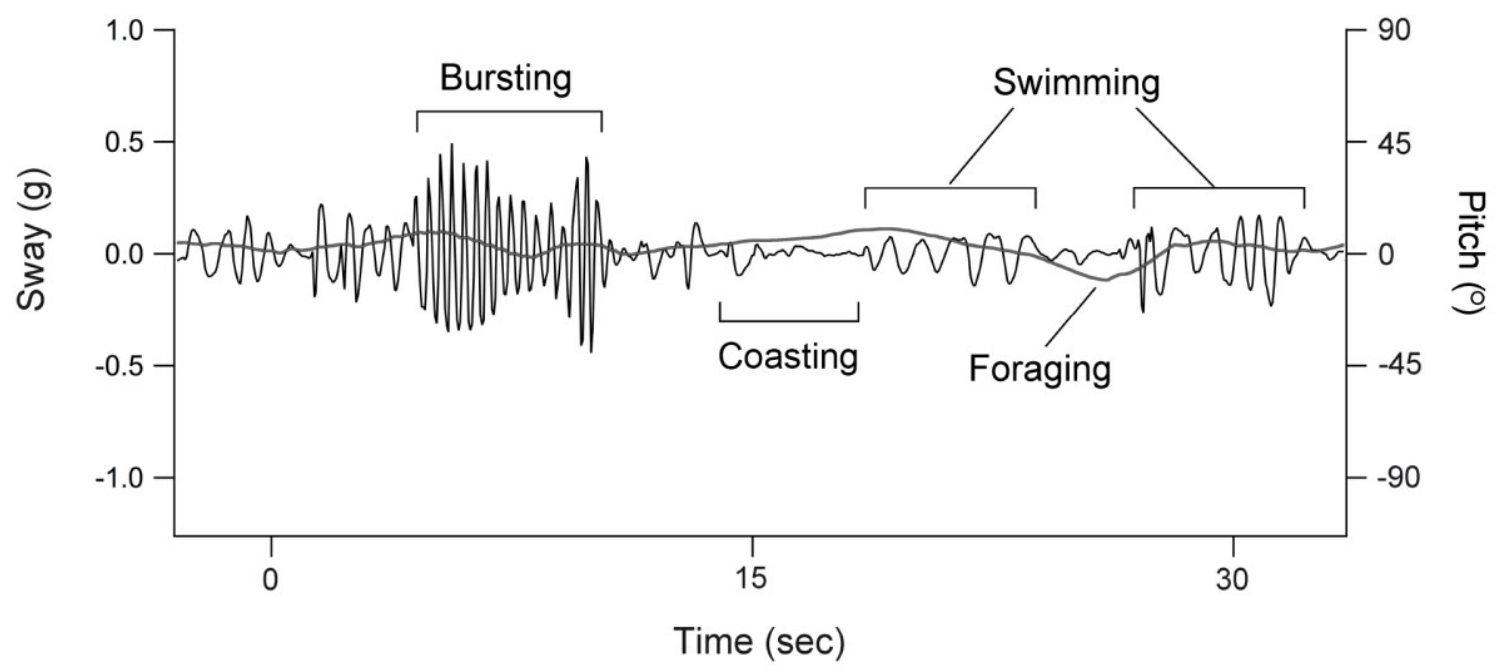


Fig. 2.3: An untagged bonefish foraging in the wetland mesocosm. Photo credit: Karen Murchie.

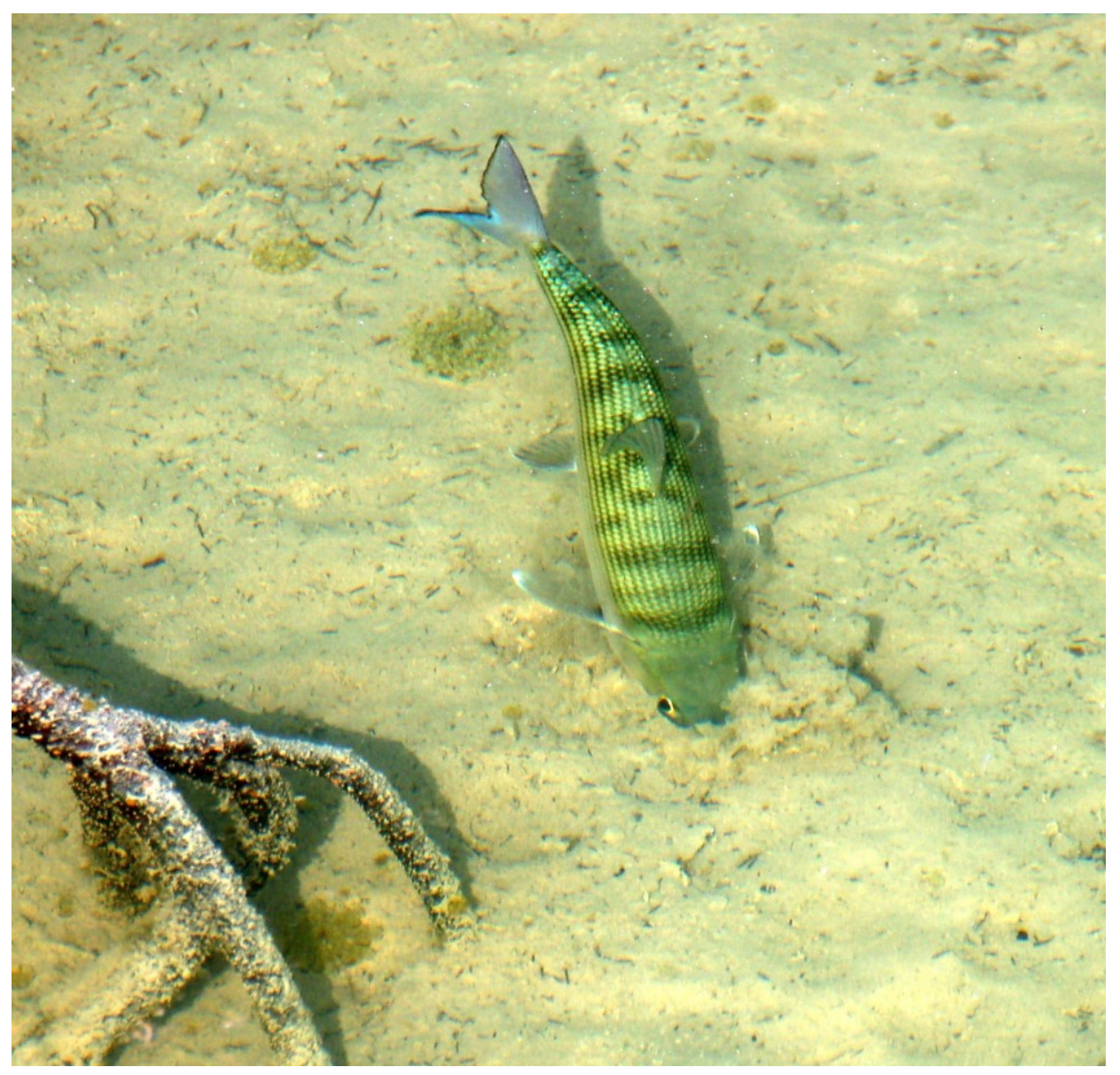


Fig. 2.4: Individual bonefish swimming behaviour (\% time), mean swimming speed $(\mathrm{m} / \mathrm{s})$, and foraging (\# events/hr) across the diel period (dawn, day, dusk, night) in the wetland mesocosm.
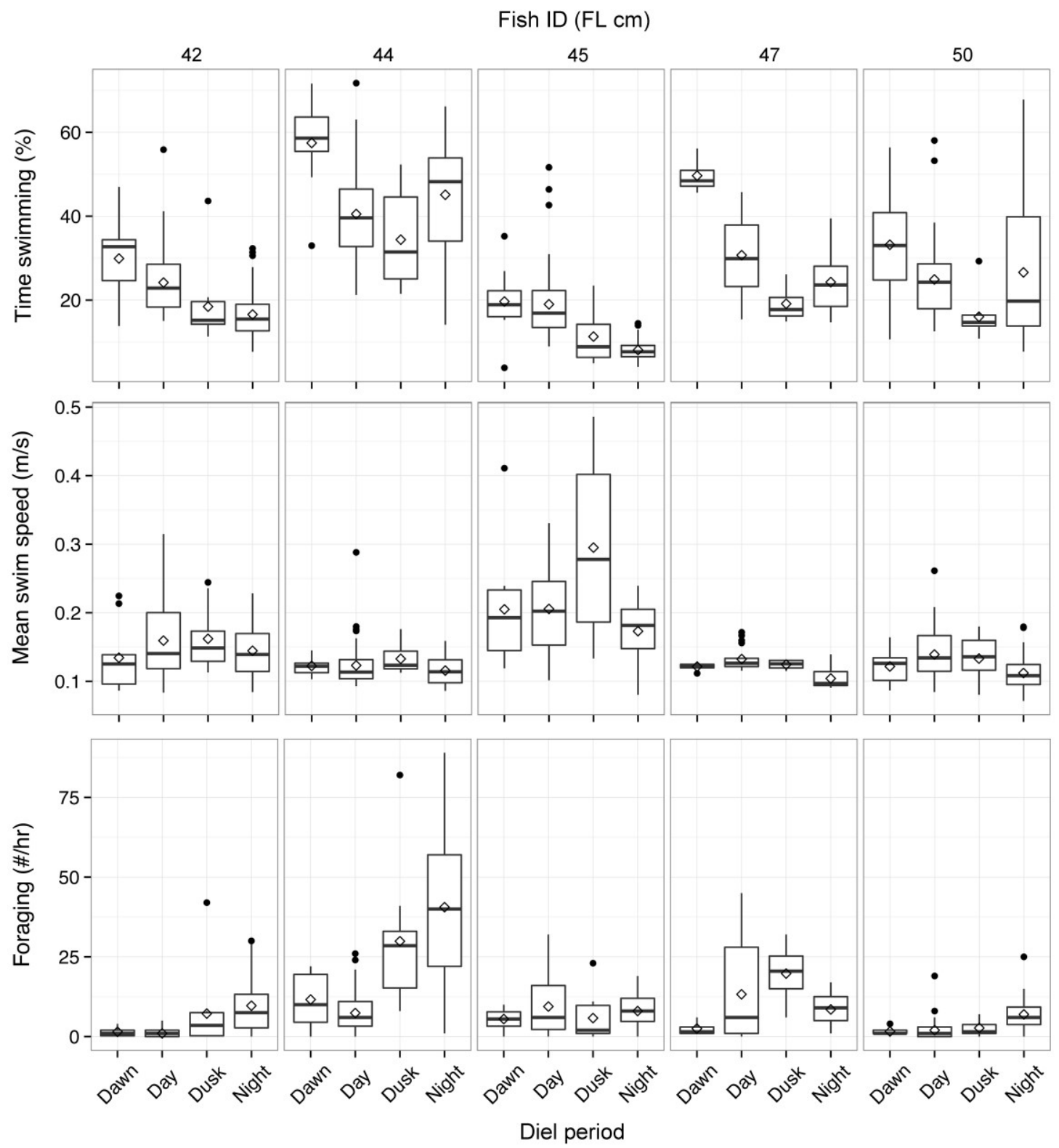
Fig. 2.5: Individual bonefish swimming behaviour (\% time) across temperature and tidal periods while in the wetland mesocosm.

Fish ID (FL cm)
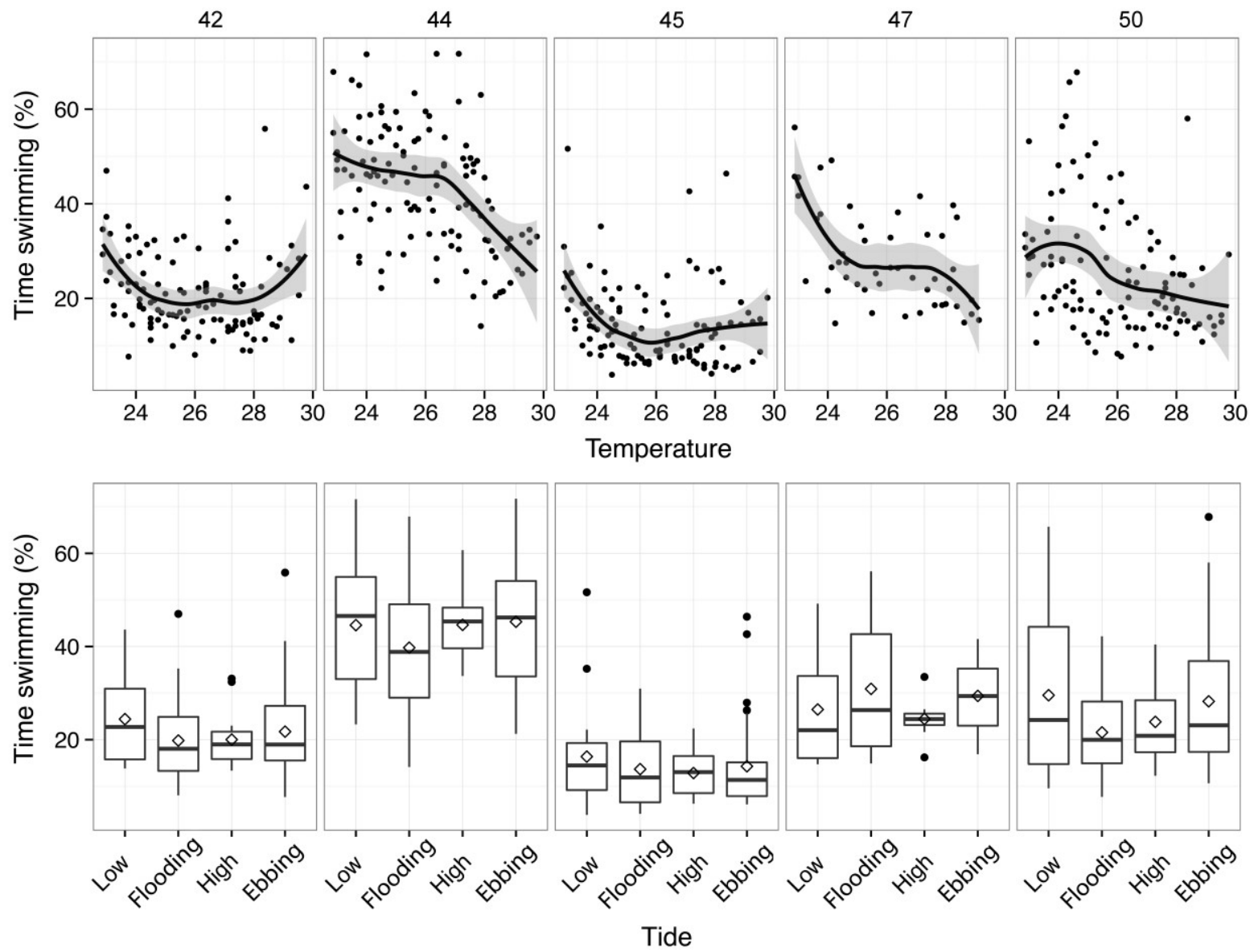
Table 2.1: Observed bonefish behaviours, corresponding accelerometrics, criteria, and identification efficiency using tri-axial accelerometer loggers. Under Criteria, Swim refers to time post-swimming, and z represents dynamic acceleration in the sway axis. All accelerometrics are presented as mean $\pm \mathrm{SD}$ (min-max). 


\begin{tabular}{|c|c|c|c|c|c|}
\hline Behaviour & Rest & Swim & Burst & Coast & Forage \\
\hline $\begin{array}{l}\text { \# Observations } \\
\text { (Duration, s) }\end{array}$ & $10(17 \pm 12)$ & $16(14 \pm 17)$ & $7(4.1 \pm 1.1)$ & $9(4.7 \pm 0.7)$ & $10(10.4 \pm 5.2)$ \\
\hline ODBA(g) & $\begin{array}{l}0.03 \pm 0.009 \\
(0.005,0.10)\end{array}$ & $\begin{array}{l}0.21 \pm 0.08 \\
(0.02,1.2)\end{array}$ & $\begin{array}{l}1.0 \pm 0.45 \\
(0.14,3.5)\end{array}$ & $\begin{array}{l}0.05 \pm 0.02 \\
(0.01,0.14)\end{array}$ & $\begin{array}{l}0.12 \pm 0.05 \\
(0.04,0.16)\end{array}$ \\
\hline $\begin{array}{l}\text { Sway } \\
\text { frequency } \\
(\mathrm{Hz})\end{array}$ & $\begin{array}{l}1.1 \pm 0.36 \\
(0.56,11.2)\end{array}$ & $\begin{array}{l}1.8 \pm 0.51 \\
(0.56,4.0)\end{array}$ & $\begin{array}{l}4.0 \pm 0.96 \\
(0.56,8.9)\end{array}$ & $\begin{array}{l}0.89 \pm 0.32 \\
(0.56,2.0)\end{array}$ & $\begin{array}{l}1.5 \pm 0.57 \\
(0.62,2.3)\end{array}$ \\
\hline $\begin{array}{l}\text { Sway } \\
\text { amplitude (g) }\end{array}$ & $\begin{array}{l}0.01 \pm 0.003 \\
(0.003,0.03)\end{array}$ & $\begin{array}{l}0.10 \pm 0.04 \\
(0.02,0.47)\end{array}$ & $\begin{array}{l}0.41 \pm 0.14 \\
(0.12,0.96)\end{array}$ & $\begin{array}{l}0.02 \pm 0.003 \\
(0.008,0.03)\end{array}$ & $\begin{array}{l}0.04 \pm 0.02 \\
(0.02,0.06)\end{array}$ \\
\hline $\begin{array}{l}\text { Swim speed } \\
(\mathrm{m} / \mathrm{sec})\end{array}$ & - & $\begin{array}{l}0.57 \pm 0.41 \\
(0.09,2.5)\end{array}$ & $\begin{array}{l}2.5 \pm 2.1 \\
(0.69,6.4)\end{array}$ & - & - \\
\hline $\operatorname{Pitch}\left({ }^{\circ}\right) \pm \mathrm{SD}$ & $\begin{array}{l}1.4 \pm 2.2 \\
(-6.5,7.7)\end{array}$ & $\begin{array}{l}4.0 \pm 1.5 \\
(-3.4,11.3)\end{array}$ & $\begin{array}{l}9.8 \pm 4.3 \\
(-1.25,31.9)\end{array}$ & $\begin{array}{l}3.4 \pm 1.5 \\
(-0.40,8.8)\end{array}$ & $\begin{array}{l}-8.7 \pm 4.5 \\
(-17.3,-4.3)\end{array}$ \\
\hline$\Delta \mathrm{Pitch}^{\circ} / \mathrm{s}$ & $\begin{array}{l}0.15 \pm 0.32 \\
(-3.3,5.1)\end{array}$ & $\begin{array}{l}-0.02 \pm 0.40 \\
(-4.9,6.2)\end{array}$ & $\begin{array}{l}0.52 \pm 0.97 \\
(-15.8,18.5)\end{array}$ & $\begin{array}{l}3.1 \pm 1.7 \\
(-0.79,8.8)\end{array}$ & $\begin{array}{l}-5.5 \pm 2.3 \\
(-9.8,-3.1)\end{array}$ \\
\hline Criteria & $\mathrm{z}<0.03 \mathrm{~g}$ & $\mathrm{z} \geq 0.03 \mathrm{~g}$ & $\mathrm{z} \geq 0.25 \mathrm{~g}$ & $\begin{array}{l}\text { Swim } \leq 5 \mathrm{~s}+ \\
\mathrm{z} \geq 0.01\end{array}$ & $\begin{array}{l}\text { Swim } \leq 4 \\
\text { sec }+\Delta \text { pitch } \\
\leq-3^{\circ}+\text { pitch } \leq \\
-4^{\circ}\end{array}$ \\
\hline $\begin{array}{l}\% \text { Correct ID } \\
(\% \text { false })\end{array}$ & $100(3.2)$ & $96(2.2)$ & $100.0(6.2)$ & $91(3.0)$ & $100(0.25)$ \\
\hline
\end{tabular}


Table 2.2: Significant factors predicting bonefish swimming behaviour ( $\%$ time $)$, mean swimming speeds $(\mathrm{m} / \mathrm{s})$, and foraging behaviour (\# events $/ \mathrm{hr}$ ) in the wetland mesocosm over a 5-day period using generalized least squares models.

\begin{tabular}{|c|c|c|c|c|}
\hline Variable & Factor & $\mathrm{DF}$ & F-value & p-value \\
\hline \multirow[t]{7}{*}{ Swim (\% time) } & Fish ID & 4,475 & 67.8 & $<0.001$ \\
\hline & Time of day & 3,475 & 21.9 & $<0.001$ \\
\hline & Temperature & 1,475 & 21.6 & $<0.001$ \\
\hline & Tide & 3,475 & 4.4 & 0.005 \\
\hline & Hour & 1,475 & 7.7 & 0.006 \\
\hline & Hour: Fish ID & 4,475 & 5.3 & $<0.001$ \\
\hline & Time of day: Fish ID & 12,475 & 2.8 & 0.001 \\
\hline \multirow[t]{3}{*}{$\begin{array}{l}\text { Mean swim } \\
\text { speed }(\mathrm{m} / \mathrm{s})\end{array}$} & Fish ID & 4,495 & 10.4 & $<0.001$ \\
\hline & Time of day & 3,495 & 5.6 & $<0.001$ \\
\hline & Hour & 1,495 & 45.8 & $<0.001$ \\
\hline \multirow[t]{6}{*}{ Foraging $(\# / \mathrm{hr})$} & Fish ID & 4,474 & 24.5 & $<0.001$ \\
\hline & Time of day & 3,474 & 28.3 & $<0.001$ \\
\hline & Hour & 1,474 & 11.7 & $<0.001$ \\
\hline & Time of day: Fish ID & 12,474 & 3.3 & $<0.001$ \\
\hline & Hour: Fish ID & 4,474 & 4.4 & 0.002 \\
\hline & $\begin{array}{l}\text { Fish ID: } \\
\text { Temperature }\end{array}$ & 5,474 & 2.3 & 0.04 \\
\hline
\end{tabular}




\subsection{Discussion}

Tri-axial accelerometers provided an effective means for quantifying bonefish behaviours (resting, swimming, bursting, coasting, and foraging) and swimming speeds. Sway amplitudes provided the best proxy for most behaviours (i.e., swimming, resting, coasting), while pitch metrics allowed for effective measurement of foraging behaviour. Similarly, tail beat metrics were also good measures of whitetip reef shark (Triaenodon obesus) swimming, resting, and even reproductive behaviours (Whitney et al. 2007), while more complex metrics have also been employed to identify a wide range of behaviours, including foraging strikes in great sculpin (Broell et al. 2013). Remote measurement of foraging behaviour is particularly challenging for animals in aquatic environments, however, accelerometers have greatly improved the ability to infer foraging behaviour. As a benthic feeder, bonefish foraging was characterized by sharp declines in pitch and minimum pitch values preceded by swimming behaviour, which were effectively measured using tri-axial accelerometer loggers. While accelerometers have been used recently to identify foraging of fish in cages or tanks (Føre et al. 2011; Broell et al. 2013), this study was, to my knowledge, the first to measure foraging behaviour of a teleost fish in a more natural environment. The ability to remotely measure foraging behaviour has important applications for understanding behavioural ecology (i.e. foraging locations and their characteristics), and energy dynamics, enabling the measurement of not only energy expenditure but also potential energy gain. It is, however, important to note that it is foraging behaviour, not necessarily successful feeding that is being measured. Future research could identify foraging success rates in 
laboratory-based observational studies, or even behavioural signatures of successful foraging events using accelerometry.

Overall dynamic body acceleration is a well-established metric for predicting locomotory speeds and energy expenditure across a wide range of taxa (Gleiss et al. 2011). Indeed, there was a strong positive correlation between bonefish swimming speed and ODBA from the swim tunnel, while recent research has shown that acceleration and swimming speeds are both strong predictors of metabolism in teleost fishes (Wilson et al. 2013; Wright et al. 2014). Active metabolism can represent a major component of an animal's energy budget (Boisclair \& Leggett 1988; Giacomini et al. 2013), and quantification of activity has applications for bioenergetics models and understanding habitat requirements. For example, further work may determine the relationship between bonefish swimming speed and metabolic rate, enabling field estimates of daily and seasonal energetic requirements using accelerometry. Indeed, tri-axial accelerometer biologgers show promise for quantifying fine scale energy dynamics (both energy expenditure and gain) of fishes in the wild. However, it is important to recognize that estimating the active metabolism of fishes based steady state movement is a simplified representation of their energetics, which vary with complex maneuvers (Boisclair \& Tang 1993).

While in the wetland, bonefish exhibited predominantly slow swimming behaviour (mean $0.18 \mathrm{~m} / \mathrm{s}$ ). However, bonefish commonly exhibited burst and coast swimming behaviour (mean of $17 \%$ of time spent coasting) with burst speeds estimated 
up to 4.2 to $6.4 \mathrm{~m} / \mathrm{sec}$ across individuals, which are similar to maximum swimming speeds estimated for sockeye salmon using EMG radiotelemetry (Hinch \& Rand 1998). In bonefish, these speeds were never observed in the confines of the swim tunnel, and these estimates are based on extrapolations from the predictive model. Nevertheless, the majority of ODBA values recorded in the wetland were within the range measured in the swim tunnel and therefore provide reliable estimates of swimming speeds, which future research could use to predict metabolism. There was also significant inter-individual variability in bonefish swimming behaviour, swimming speeds, and foraging behaviour in the wetland. Animal behaviour in general is often highly variable within species due to genetic, ontogenetic, physiological, or experiential factors, but individuals commonly exhibit consistent behavioural types (personalities; Sih et al. 2004; Dall et al. 2004). Such individual variability may have important ecological (e.g., population dynamics, environmental interactions) and evolutionary (e.g., evolvability, speciation) implications, and on a population level, may be important for survival in changing environments (Wolf \& Weisseng 2012). The sample size of this study $(n=5)$ has revealed how variable bonefish behaviour can be between individuals, and larger sample sizes may indicate the relative prevalence of these behavioural types in the population.

Despite the high inter-individual variability, bonefish behaviours showed some consistent patterns across environmental factors. Diel period was a strong predictor of all the aforementioned bonefish behaviours, while temperature and tidal period also explained swimming behaviour. Both light and temperature often have strong influence on biological rhythms in fish (Zhdanova \& Reebs 2006), while tides are important drivers 
of behaviour in species that live in near shore marine ecosystems (Gibson 1986; Krumme 2009). While all of these factors may influence bonefish behavior, it is impossible to untangle the effects of light, temperature, and tide on bonefish behaviour in the wetland mesocosm over a 5-day period. As the technology of accelerometer loggers advances, longer-term studies in the wild should enable observations across a greater range of environmental parameters (i.e. across entire tidal periods) and increase the ability to identify cues for certain behaviors in bonefish.

Across the diel cycle, the majority of bonefish were most active (swimming) at dawn and during daylight hours, however some fish were also highly active at night. Light intensity often affects fish behaviour because it influences their ability to forage, rest, avoid predators, aggregate, or reproduce (Helfman 1986; Zhdanova \& Reebs 2006). It is interesting that although bonefish exhibited foraging behaviour throughout the diel cycle, most foraging behaviour was detected at night (Fig. 2.4). Given that bonefish were not directly observed during the night period, it is possible that bonefish exhibit behaviours unobserved during the day that have a similar accelerometric signal (e.g. sleep-like state). However, it is certainly plausible that bonefish foraged most often night. In the wetland, bonefish were actively swimming during the night periods, and fish that were highly active swimming at night also exhibited high frequencies of foraging behaviour (e.g., fish 44). Further, in the wild, bonefish often migrate into near shore tidal areas during nighttime hours, a migration that is associated with foraging (Colton \& Alevizon 1983b; Murchie et al. 2013). 
Bonefish spent the most time swimming while temperatures were lowest ( $\sim 22$ to $25^{\circ} \mathrm{C}$; coinciding with early morning to early day), and became less active as temperatures increased during the day and into the evening hours. They also exhibited the highest mean swimming speeds during daytime and evening hours when temperatures were highest ( 26 to $30^{\circ} \mathrm{C}$; Fig. 2.5). Preliminary data on bonefish thermal biology suggests that maximum aerobic scope occurs at approximately $26-28^{\circ} \mathrm{C}$, while in winter months (i.e. during this study), preferred temperatures are $\sim 24^{\circ} \mathrm{C}$ (Nowell et al. 2015). It is not surprising that bonefish were most active at their preferred temperatures, which they were likely acclimatized to in the wild. Higher swimming speeds at higher temperatures may reflect higher aerobic scope, or that bonefish are seeking more optimal temperatures. Indeed, bonefish live in environments with high thermal variability and strong thermal gradients where behavioral thermoregulation can occur on small spatial scales (Murchie et al. 2011b).

Over the tidal cycle, bonefish were generally most active during ebbing and low tides, and least active at flooding, although the differences in activity were relatively small. Interestingly, water levels in the wetland mesocosm were not directly influenced by tides because although the wetland is adjacent to the ocean, it is on a higher elevation and typically disconnected. Bonefish typically reside in shallow, near-shore seas that are tidally influenced, and make feeding migrations with flooding tides (Colton \& Alevizon 1983b; Murchie et al. 2013). Many species that live in these areas have sensory systems that detect changes in pressure associated with changing tides (Gibson 1986). Bonefish may have sensed declines in pressure associated with ebbing tides that triggered activity 
that would normally be required to leave near shore feeding areas with ebbing tides. Indeed, responses to tides may be an entrained biological rhythm despite the fact that water levels are static. Future studies on bonefish in areas under direct tidal influence are needed to better understand the influence of tides on their behaviour.

In summary, tri-axial accelerometers provided a reliable means for measuring a range of bonefish behaviours and swimming speeds, and when applied to bonefish in a wetland mesocosm, revealed high inter-individual variability, and potential influences of environmental factors including diel period, temperature, and tide on bonefish behaviour. The ability to measure fine-scale behaviour such as foraging may identify habitat requirements and inform bioenergetics models, providing insight into population dynamics, mechanisms of animal behaviour, and energy landscapes. To this end, further work should estimate the relationship between bonefish swimming speeds and energy expenditure, the relative energetic costs of active metabolism and digestion, and foraging efficiency. Given the relationship between energy acquisition, growth and reproductive investment, the ability to study feeding ecology in free-ranging fish provides scientists with new opportunities for tracking fitness. 


\section{Chapter 3. Spatiotemporal drivers of energy expenditure in a coastal marine fish}

\subsection{Abstract}

Animal behaviour and energy expenditure often vary significantly across the landscape, and quantifying energy expenditure over space and time provides mechanistic insight into ecological dynamics. However, spatiotemporal variability in energy expenditure has rarely been explored in fully aquatic species such as fish. Our objective was to quantify spatially explicit energy expenditure for a tropical marine teleost fish, bonefish (Albula vulpes), to examine how bonefish energetics vary across landscape features and temporal factors. Using a swim tunnel respirometer we calibrated acoustic accelerometer transmitters implanted in bonefish to estimate their energy expenditure, and applied this technology to estimate bonefish energy expenditure in situ using a fine-scale telemetry system on a heterogeneous reef flat in Puerto Rico. Bonefish energy expenditure varied most significantly across habitats, with significant interactions between habitat and temporal factors (i.e., diel period, tide state, season). Energy expenditure was generally highest in shallow water habitats, during the day, and flowing tides (flooding and ebbing). Variation in activity levels was the main driver of differences in energy expenditure; higher energy expenditure in shallow water habitats was likely related to foraging. Bonefish moderate energy expenditure across seasonal fluctuations in temperature by selectively using shallow near shore habitats to avoid thermal extremes. Quantifying how animals expend energy in association with environmental and ecological factors can 
provide important insight into ecological dynamics, with implications for both behavioural ecology and bioenergetics models.

\subsection{Introduction}

Energy is considered the fire of life, acquired by organisms by consumption and expended for metabolic maintenance, activity, growth, and reproduction (Kleiber 1961; Tytler \& Calow 1985). It is a key currency by which we can measure individual fitness, as well as population and ecosystem dynamics (Brown et al. 2004). Ultimately, organisms must acquire energy at a greater rate than it is expended to allocate resources to growth and reproduction; yet tradeoffs must be made amongst various fitnessenhancing activities. For example, time and energy spent on predator avoidance often comes at the expense of foraging (Milinski \& Heller 1978; Hugie \& Dill 1994; Lind \& Cresswell 2005). Animal energetics may also vary due to spatiotemporal factors such as the distribution of resources, costs of movement, temperature, intraspecific competition, and predation risk (Lima \& Dill 1990; Johnson et al. 1992; Lima \& Zollner 1996; Hall \& Clark 2016). The field of ecological energetics seeks to understand how ecological factors influence animal bioenergetics, with implications for fundamental ecology and predicting the impacts of anthropogenic stressors (Tomlinson et al. 2014)

Bioenergetics models play a key role in understanding how animals make a living and allocate resources (Kleiber 1961; Harfmart \& Brandt 1995). Active metabolism (i.e. energy used for movement) often constitutes a significant portion of energy budgets 
(Boisclair \& Leggett 1989; Boisclair \& Sirois 1993), but can be highly variable in the wild due to the influence of ecological factors on animal behaviour and the energy expenditure (Houston et al. 1993; Brown et al. 2004; Shepard et al. 2013). Despite the fact that bioenergetics models are the most widely developed and applied in fish of any taxa (e.g. Hansen et al. 1993; Hartman and Brandt 1995), variation in energy dynamics due to ecological factors in the wild are rarely integrated into estimates of metabolism (but see Payne et al. 2011; Brodie et al. 2016). Most empirical studies on wild fish bioenergetics either study on fish in the laboratory, or examine fish movement in relation to ecological factors (e.g., temperature), using growth or reproductive output as endpoints (e.g., Pauly 1980; Jobling 1995; Wurtsbaugh \& Neverman 1988). Yet, energy expenditure is ultimately dependent on fish behaviour. For example, fish often behaviourally thermoregulate (Holland et al. 1992), or use specific water flows to minimize energy expenditure (Taylor et al. 2014; McElroy et al. 2012). With recent advances in biotelemetry and biologging technology, we can now acquire more direct estimates of energy expenditure, and potentially even energy gain (Cooke et al. 2016), enabling opportunities to quantifying how fish expend energy in relation to ecological factors, providing greater insight into their spatial ecology, population dynamics, and improved estimates for bioenergetics models.

Here I examine how ecological factors influence the energy expenditure of a benthivorous marine teleost fish, bonefish (Albula vulpes). Bonefish are a group of fishes that occupy shallow tropical and subtropical seas worldwide in diverse habitats including seagrass, sand flats, and coral reefs, moving frequently between deep-water habitats into 
shallow nearshore areas with the tides (Humston et al. 2005, Danylchuk et al. 2011, Murchie et al. 2013). I used a swim tunnel respirometer to calibrate acoustic accelerometer transmitters to estimate energy expenditure with bonefish, and applied this technology to quantify energy expenditure with a fine scale positioning system on a heterogeneous reef flat in Culebra, Puerto Rico. In doing so, I aimed to provide some of the most extensive estimates of the influence of ecological factors on energy expenditure in a teleost fish.

\subsection{Methods}

\subsubsection{Accelerometer calibration}

In order estimate bonefish energy expenditure in the wild, I used Vemco (Vemco Inc., Shad Bay, NS) acceleration acoustic transmitters (V13A, $12.2 \mathrm{~g}$ in air, $35 \mathrm{~s}$ transmission delay, $\pm 3.43 \mathrm{~g}$ acceleration range, $5 \mathrm{~Hz}$ sampling frequency). These transmitters function by measuring acceleration $\left(\mathrm{g}, 9.8 \mathrm{~m} \mathrm{~s}^{2}\right)$ in three axes ( $\mathrm{x}=$ lateral, $\mathrm{y}=$ forward, and $\mathrm{z}=\mathrm{vertical}$ ) and processing the information before transmission. The root mean square (RMS) of acceleration (a) is calculated as: $a=\sqrt{A_{x}^{2}+A_{y}^{2}+A_{z}^{2}}$ where A represents acceleration in each axis. The RMS is averaged over each sampling period ( $25 \mathrm{~s})$, and stored in memory until transmission.

I calibrated accelerometer transmitters to predict mass-specific metabolic rate $\left(\mathrm{MO}^{2} ; \mathrm{O}_{2} \mathrm{~min}^{-1} \mathrm{~kg}^{-1}\right)$ in bonefish using a Blazka-style flow through respirometer swim 
tunnel at the Cape Eleuthera Institute (CEI) in Eleuthera, The Bahamas using a two-step experimental approach, by first calibrating acceleration with swimming speed, and then swimming speed to oxygen consumption. It is well established that the dynamic acceleration generated by animals is highly correlated with the rate of movement and metabolism in a wide range of species from diverse taxa (Halsey et al. 2011), including fish (Gleiss et al. 2010; Wilson et al. 2013; Wright et al. 2014). Firstly, I determined the relationship between transmitter-derived acceleration and bonefish swimming speed. Bonefish $(\mathrm{n}=10 ; 42 \pm 6 \mathrm{~cm}$ fork length; mean $\pm S D$; range $=32-53 \mathrm{~cm})$ were captured from a tidal mangrove creek in Eleuthera via seine net on January 102014 and transported back to a wet laboratory facility at CEI (as per methods in Murchie et al. 2009). Fish were held in a large circular tank (3.7 m diameter $\times 1.25 \mathrm{~m}$ height; $13180 \mathrm{l})$ for 3-7 days prior to experimentation, and were fed daily rations of cut fish to satiation. Twenty-four hours prior to experimentation, V13AP transmitters were surgically implanted in bonefish as per the methods described above. Bonefish were then placed in a Blazka-style swim tunnel (24.1 internal diameter $\times 116 \mathrm{~cm}$ length; see Thorstad et al. 1997 for additional details) and given $1 \mathrm{hr}$ prior to the swimming protocol to acclimate to the tunnel. Water flow was increased to $10 \mathrm{~cm} / \mathrm{sec}$ until the bonefish maintained its position in the tunnel (swimming the same speed as the flow) for at least three successful data transmissions. Swimming speeds were increased by $15 \mathrm{~cm} / \mathrm{s}$ thereafter following the same protocol. Flow speed was increased until the fish could no longer maintain its position in the tunnel, i.e., when its tail was touching the back grate for $>5 \mathrm{~s}$. Bonefish were then transferred to a holding tank to recover for $24 \mathrm{hr}$, when transmitters were 
surgically removed. Bonefish were held for another $24 \mathrm{hr}$ for recovery and released back into the wild.

The experiments conducted to estimate the relationship between bonefish swimming speed and $\mathrm{MO}_{2}$ were reported in Nowell et al. (2015). Briefly, bonefish ( $\mathrm{n}=$ $66 ; 41 \pm 4 \mathrm{~cm}$ fork length; $847 \pm 257 \mathrm{~g}$; mean $\pm \mathrm{SD}$; range $=34-51 \mathrm{~cm})$ were subjected to a ramp-Ucrit procedure (Jain et al. 1997) involving $15 \mathrm{~cm} / \mathrm{s}$ increases in swimming speed every 15 min until exhaustion. Oxygen consumption was measured at each speed over a 10 min period with an OxyGuard oxygen probe (OxyGuard Handy Polaris 2, portable DO meter, Water Management Technologies, Inc. Baton Rouge, LA, USA). Swimming trials were run from Oct-2012 to Nov-2013, using natural seasonal variations in water temperature to collect metabolic estimates from a range of temperatures bonefish experience in the wild, $22-36^{\circ} \mathrm{C}$, with a range of sizes at each temperature (see Nowell et al. 2015 for more details).

\subsubsection{Study site and habitat mapping}

The field study was conducted in Culebra, Puerto Rico on a fringing coral reef, Las Pelas $\left(18.2966^{\circ} \mathrm{N},-65.2500^{\circ} \mathrm{W}\right)$. In order to characterize habitats in the study area, depth, abiotic and biotic conditions were quantified at 487 locations along four transects spanning beyond estimated bonefish locations in all directions (See Supplementary Material, Fig. 1 for locations). At each location, the percentage benthic cover of substrate (sand, coral rubble, or sand/fine sediment), seagrass (Thalassia testudinum, Syrungodium 
filliforme, Halodule wrightii), macro algae (Halimeda spp., Penicillus spp), and coral (dead hard coral, live hard coral, or live soft coral, Diploria, Millepora, Gorgoniidae) were visually estimated within a $1-\mathrm{m}^{2}$ quadrat. Using these samples, five distinct habitat types were identified with a k-means clustering algorithms to minimize the sum of squares. These included forereef - mainly live and dead hard corals and coral rubble, reef crest - primarily coral rubble and sand, seagrass - seagrass with sparse macro algae and sand, transition - mainly sand with some macro algae and seagrass, and lagoon sand/soft sediment with sparse macro algae (Fig. 3.1). The spatial extent of each habitat was then determined using a semi-automatic classification algorithm in QGIS version 2.10.1 (Free Software Foundation, 1991) using Google Earth version 7.1.5.1557 satellite imagery (Google Earth, 2015). Known habitat types from habitat sampling locations were used in the training data set.

Depth was also measured at a subset $(\mathrm{n}=107)$ of the habitat sampling locations using a Hawkeye D10 Depth Sounder (NorCross Marine Products, Inc, USA) for locations $>1 \mathrm{~m}$ in depth, or a measuring tape at locations $<1 \mathrm{~m}$. Time was also recorded to correct for tide height at the time of sampling. Depths were estimated throughout the study region using kriging interpolation. Mean depth in the lagoon was $4.83 \mathrm{~m}$, transition $1.40 \mathrm{~m}$, seagrass $0.73 \mathrm{~m}$, reef crest $0.70 \mathrm{~m}$, and coral reef $2.65 \mathrm{~m}$. 


\subsubsection{Acoustic tracking system and fish tagging}

To quantify spatially explicit bonefish energy expenditure on Las Pelas, a fine scale acoustic telemetry array (i.e., Vemco Positioning System, VPS) was deployed in Aug2012, consisting of 25 VR2W receivers (69 kHz, Vemco Inc.) and 15 acoustic transmitter synchronization tags (Vemco V13-1x, 500-700 transmission delay) (Fig. 3.1). The synchronization tags were used to maintain time synchronization in the tracking system, as well as provide estimates of positioning error from known locations. Based on initial range tests indicating low detection range in this shallow, noisy system, receivers were spaced apart by $50 \mathrm{~m}$ to ensure overlapping detection ranges. The system was established to track bonefish, permit (Trachinotus falcatus), great barracuda (Sphyraena barracuda), and green sea turtles (Chelonia mydas) from Aug-2012 to Dec-2014. Based on preliminary observations of temperature differences between the forereef and backreef habitats, water temperature was measured once per hour in two locations, one on the forereef, and the other on the transition habitat using temperature loggers (Hobo Pendant UA-002-64, Onset Computer Corp, Onset MA, USA).

The bonefish examined here $(\mathrm{n}=10,52.1 \pm 6.1 \mathrm{~cm} ;$ mean $\pm \mathrm{SD} ; 41-60 \mathrm{~cm}$ range $)$ were tagged via surgical implantation in the coelomic cavity with Vemco accelerationpressure acoustic transmitters (V13AP, $12.2 \mathrm{~g}, 45-135 \mathrm{~s}$ transmission delay, $\pm 3.43 \mathrm{~g}$ acceleration range, 323 day battery life, $5 \mathrm{~Hz}$ sampling frequency) from 27-Apr-2013 to 22-May-2013. Fish were captured by fly or spin fishing on the reef crest and seagrass habitats of Las Pelas. Upon capture, fish were held in a floating mesh net pen 
$(1.5 \times 1.5 \times 1.5 \mathrm{~m})$ for at least $20 \mathrm{~min}$ prior to the tagging procedure. Bonefish were anesthetized with $100 \mathrm{ppm}$ tricaine methanesulfonate (MS-222), placed dorsal side down on a surgery table and supplied with recirculating seawater containing a maintenance does of 50 ppm MS-222 (as per Murchie et al. 2011a). A 2-3 cm incision was made with a scalpel $\sim 2 \mathrm{~cm}$ from the ventral midline of the fish posterior to the pectoral fins, and transmitters were implanted into the coelomic cavity with the sensors facing anteriorly. The incision was closed with 2-3 interrupted sutures using absorbable monofilament suture materials (Ethicon 3-0 PDS II, Johnson and Johnson, New Jersey). Bonefish were held in the net pen for a minimum of $1 \mathrm{hr}$ prior to release due to concerns about post release predation with this species (see Danylchuk et al. 2007a).

\subsubsection{Data analysis}

For the swim tunnel accelerometer transmitter experiment, bonefish acceleration was square root transformed and fit with a linear mixed effects (LME) model with swimming speed as a fixed effect and individual fish as a random effect. For the respiration swim

tunnel data, bonefish $\mathrm{MO}_{2}\left(\mathrm{mg} \mathrm{O} 2 \mathrm{~min}^{-1} \mathrm{~kg}^{-1}\right)$ was square root transformed and fit with an LME with swimming speed, fish fork length, temperature, and the interaction between swimming speed and temperature as fixed effects and individual fish as a random effect.

Detections of acoustically tagged bonefish by at least three acoustic receivers in the Vemco positioning system in Culebra were used by Vemco to estimate fish positions using hyperbolic positioning (Smith 2013). Estimated bonefish positions with associated 
acceleration data $(\mathrm{n}=60,400)$ were filtered by a maximum Horizontal Positioning Error (HPE) of ten, following the methods of Smith (2013), which retained $83 \%$ of detections. This error estimate is not spatially explicit, but in synchronization tags, applying this maximum HPE value filter resulted in a mean error of $1.2 \pm 2.2 \mathrm{~m} \mathrm{SD}$. For each fish, the initial 24 hours of data were also excluded from the data set. Retained acceleration values were used to estimate bonefish oxygen consumption $\left(\mathrm{MO}_{2}\right)$ using the two models described above. Temperature was derived from the spatially and temporally nearest environmental temperature logger to the fish. Rates of oxygen consumption $\left(\mathrm{mg} \mathrm{O}_{2} \mathrm{~min}^{-1}\right.$ $\left.\mathrm{kg}^{-1}\right)$ were converted to energy expenditure $\left(\mathrm{J} \mathrm{min}^{-1} \mathrm{~kg}^{-1}\right)$ assuming $1 \mathrm{mg} \mathrm{O} \mathrm{O}_{2}$ equated to 14.1 J of energy expended, the standard for ammoniotelic fishes (Elliot \& Davidson 1975). Bonefish positions were then aggregated into $10 \mathrm{~m}^{2}$ cells, and mean energy expenditure was calculated for each cell, fish, season, diel period, and tide state combination. These data were modeled using a linear mixed effects model with fish fork length, habitat, season, diel period, tide state, as well as habitat*season, habitat*diel period, habitat*tide state, and diel period*tide state interactions, and individual fish as a random effect. Water temperatures where bonefish were present within the tracking system were compared to all measured temperatures during the study period using a generalized linear mixed effects model (GLMM) with a poisson link function, data type (fish, environment) as a predictor and season nested in data type as a random effect.

All analyses were conducted using RStudio version 0.99.473 (RStudio Team, 2015), and R (R Core Team, 2013). Models were fit using the R packages 'nlme' (Pinheiro et al. 2015) and 'Ime4' (Bates et al. 2015). Model selection was conducted 
using the drop1 command to compare full models to reduced models with log ratio tests. Models were validated using the procedure outlined in Zuur et al. (2009). 
Fig. 3.1: Las Pelas fringing reef in Culebra, Puerto Rico. Colours represent habitat types identified using a k-means clustering algorithm based on habitat samples, and predicted across the entire area using a maximum likelihood classification algorithm on satellite imagery. Red circles represent acoustic receiver locations, and black crosses synchronization tags, comprising the Vemco Positioning System.

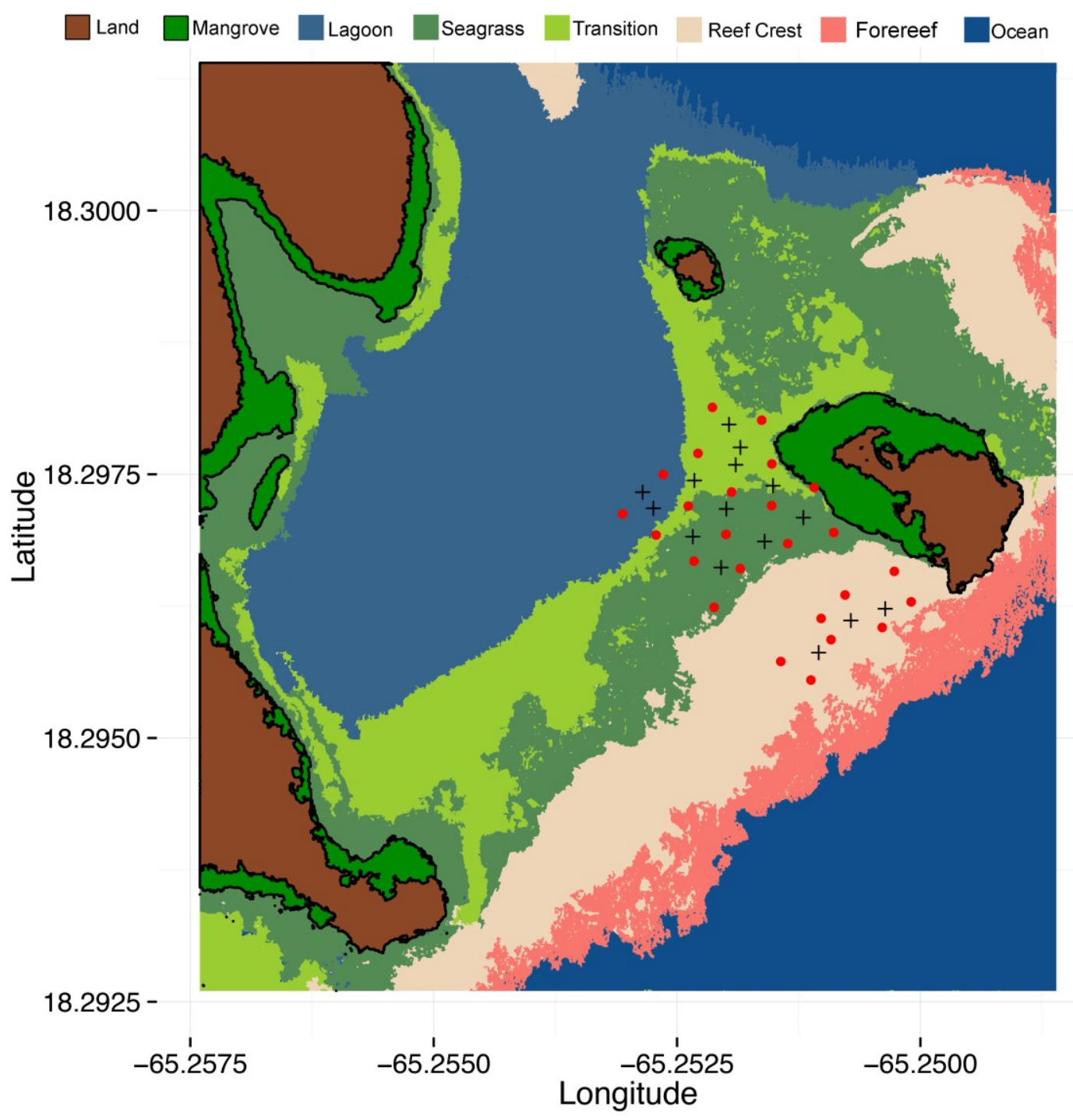




\subsection{Results}

\subsubsection{Accelerometer calibration}

There was a significant positive relationship between accelerometer transmitter-derived bonefish acceleration (ACC) and swimming speed (SS) in the swim tunnel (Table 3.1; Fig. 3.2), described by Equation 1:

1. $\mathrm{SS}=(\sqrt{\mathrm{ACC}-0.57) / 0.013}$

With untagged bonefish, swimming speed, fork length (FL), water temperature (T) and the swimming speed*temperature interaction were all significant predictors of oxygen consumption in the swim tunnel (Table 3.1; Fig. 3.2), described by Equation 2:

2. $\sqrt{ } \mathrm{MO} 2=1.16+0.03 * \mathrm{SS}-0.02 * \mathrm{FL}+0.05 * \mathrm{~T}+0.0005 * \mathrm{SS}: \mathrm{T}$

\subsubsection{Spatial bioenergetics}

The fine scale spatial positioning system on Las Pelas provided 48,593 reliable positions with acceleration estimates from ten bonefish from 30-04-2013 to 20-03-2014. There was significant variability in bonefish energy expenditure across fish sizes, habitats, and temporal factors, with significant interactions between temporal factors (diel period, tide state, season) and habitat (Table 3.2). The overall mean rate of energy expenditure was $73 \mathrm{~J} \mathrm{~min}^{-1} \mathrm{~kg}^{-1}$, and smaller bonefish generally had higher mass-specific rates of energy expenditure than larger bonefish (Fig. 3.3A). 
Across the aquatic landscape, bonefish expended more energy in shallower habitats, with the highest rates on the reef crest, particularly at night (Fig. 3.3B; Fig. 3.4). Differences in energy expenditure among tides were less pronounced than across habitats, but energy expenditure was generally higher during flooding and ebbing tides (Fig. 3.3C,D). Energy expenditure was very consistent in the lagoon across seasons, but was moderately higher in the summer and fall in other habitats (Fig. 3.3C).

Due to the nature of the predictive models developed in the swim tunnel experiments, fish length, temperature, and acceleration were intrinsically related to the estimates of bonefish energy expenditure. However, examining the factors that contributed to energy expenditure in the wild, activity (acceleration) had a greater influence over energy expenditure than temperature due to the relatively narrow range of temperatures experienced (Fig. 3.5A). Bonefish activity levels increased with temperature, but declined above $30^{\circ} \mathrm{C}$ (Fig. 3.5B). Water temperatures were also significantly different when bonefish were present in the area from the temperatures available $(\mathrm{z}=4.81$, $\mathrm{p}<0.001)$ indicating that bonefish used this shallow nearshore area selectively based on temperature (Fig. 3.6). During winter and spring, bonefish occupied this area during warmer water temperatures than available, while in summer and fall they were present at relatively colder temperatures. 
Fig. 3.2: A) Bonefish swimming speed $\left(\mathrm{m} \mathrm{s}^{-1}\right)$ and acceleration $\left(\mathrm{m} \mathrm{s}^{-2}\right)$ measured with V13AP transmitters in a swim tunnel and fitted linear mixed effects model predictions \pm 95\% CI, B) Bonefish swimming speed $\left(\mathrm{m} \mathrm{s}^{-1}\right)$ and metabolic rate $\left(\mathrm{MO}_{2} ; \mathrm{mg} \mathrm{O}_{2} \mathrm{~min}^{-1} \mathrm{~kg}^{-}\right.$ ${ }^{1}$ ) and linear mixed effect model predictions for a $61 \mathrm{~cm}$ fork length bonefish at $22^{\circ} \mathrm{C}$ (solid line), and a $34 \mathrm{~cm}$ fish at $36^{\circ} \mathrm{C}$ (hatched line) $\pm 95 \%$ CIs.
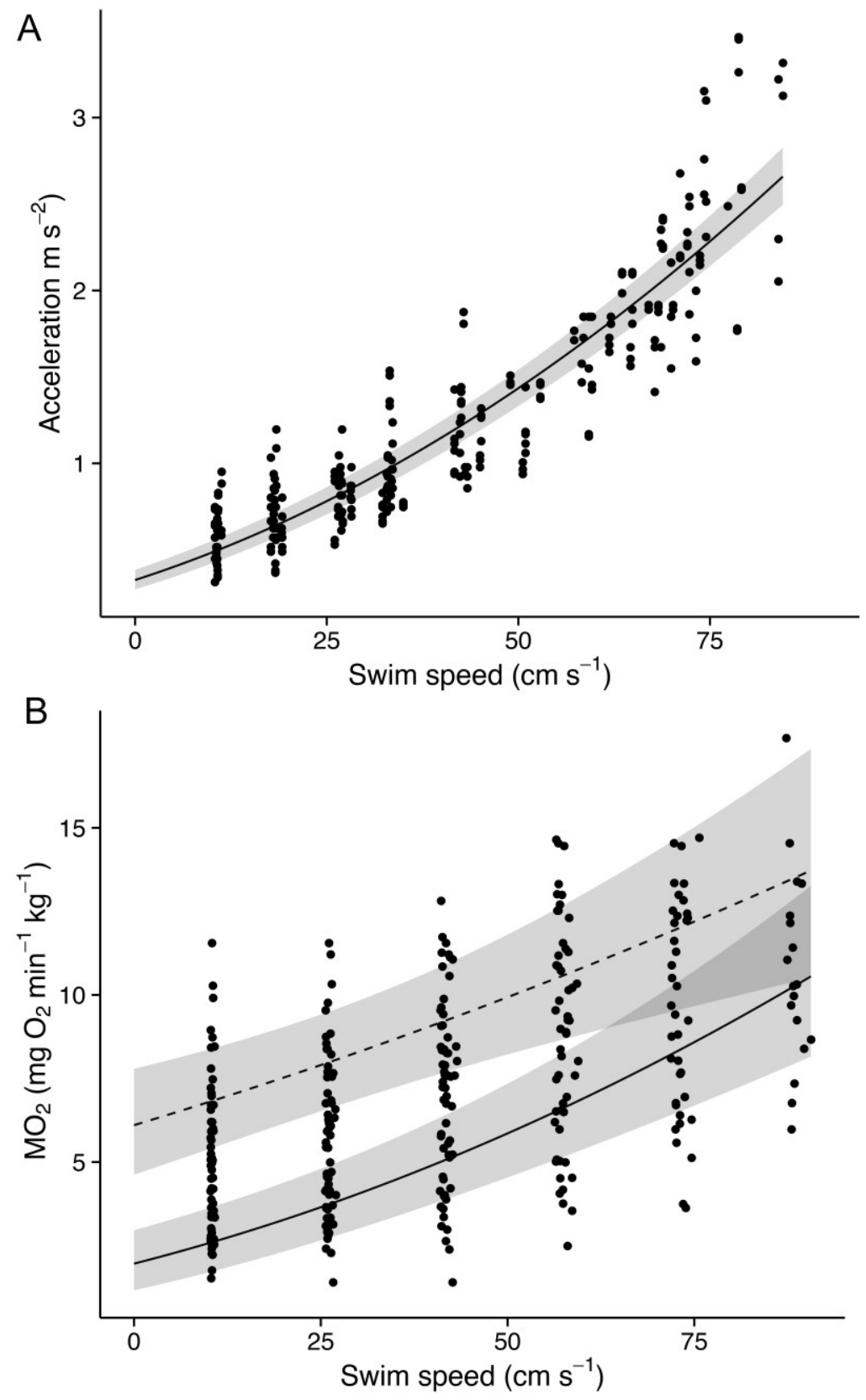
Fig. 3.3: Linear mixed effects model predictions $\pm 95 \%$ CIs of bonefish energy expenditure $\left(\mathrm{J} \mathrm{min}^{-1} \mathrm{~kg}^{-1}\right)$ by A) bonefish fork length, B) habitat type and diel period, C) habitat type and tide state, D) habitat type and season.
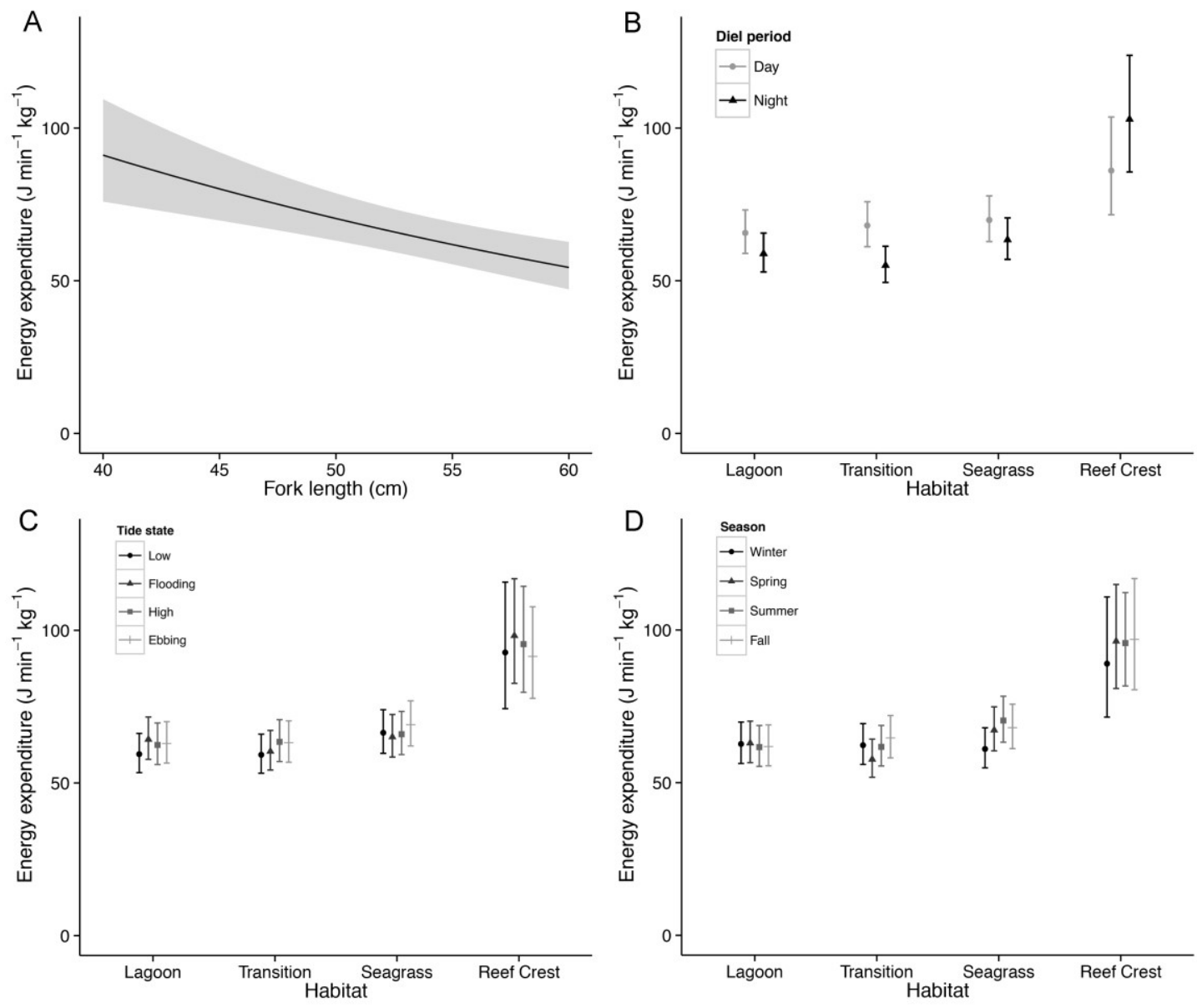
Fig. 3.4: Bonefish energy expenditure $\left(\mathrm{J} \mathrm{min}^{-1} \mathrm{~kg}^{-1}\right)$ on Las Pelas reef flat during each diel period.

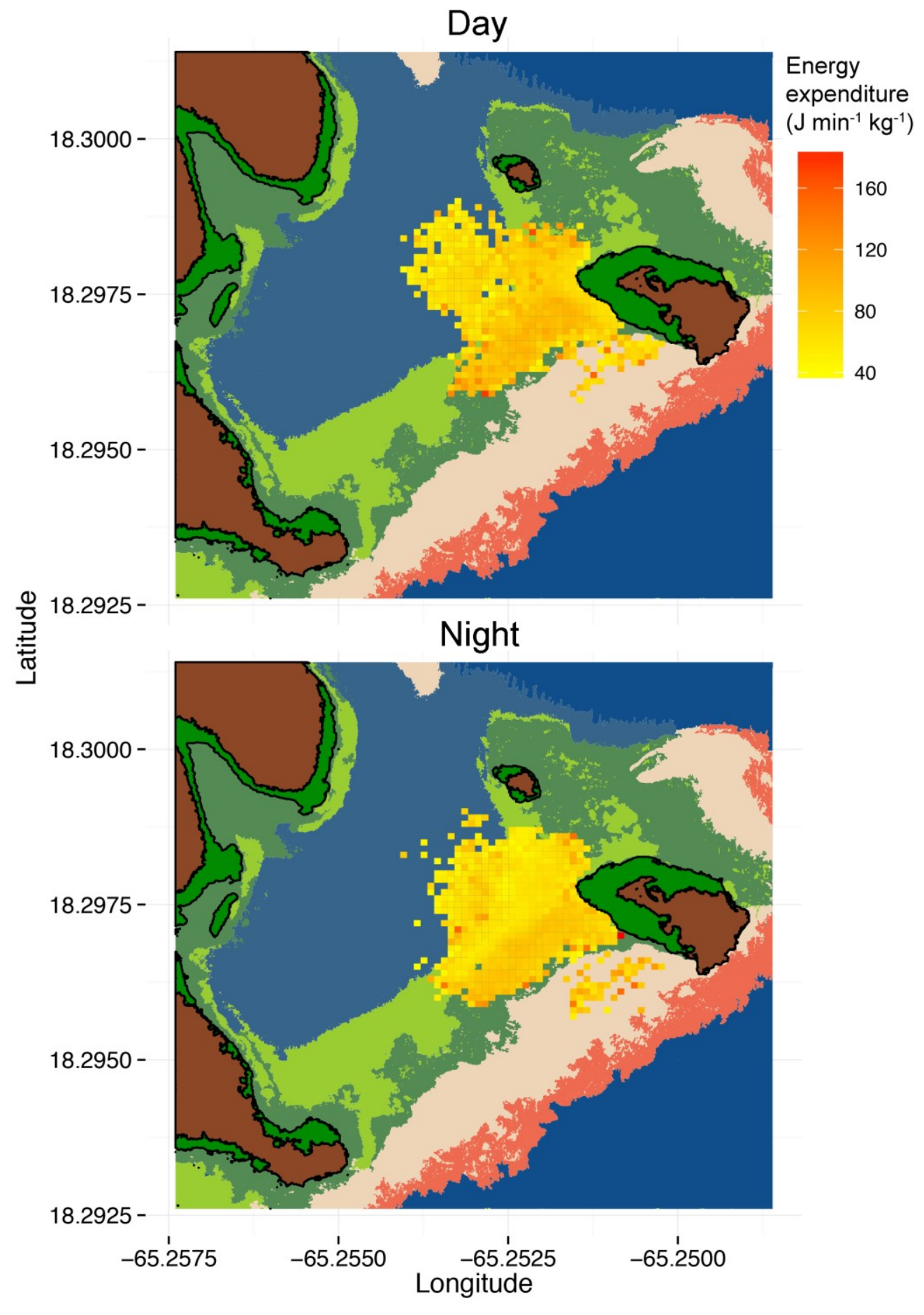


Fig. 3.5: A) Bonefish acceleration $\left(\mathrm{m} \mathrm{s}^{-2}\right)$ and energy expenditure $\left(\mathrm{J} \mathrm{min}^{-1} \mathrm{~kg}^{-1}\right)$ across a range of water temperatures, B) Water temperature $\left({ }^{\circ} \mathrm{C}\right)$ and bonefish activity (acceleration, $\mathrm{m} \mathrm{s}^{-2}$ ).
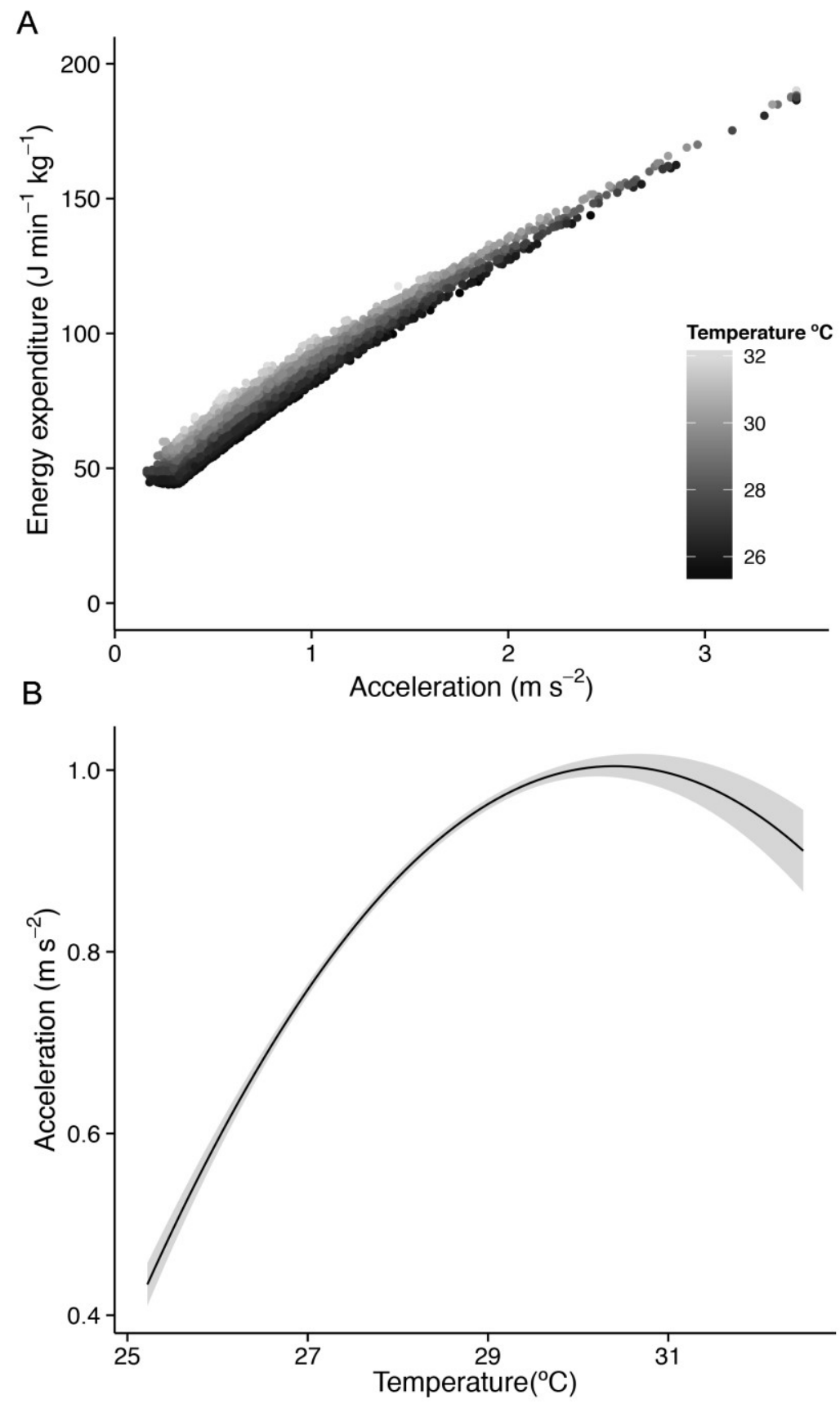
Fig. 3.6: Frequency of water temperatures in Las Pelas when tagged bonefish were present (white bars), and overall during the study period (grey bars).

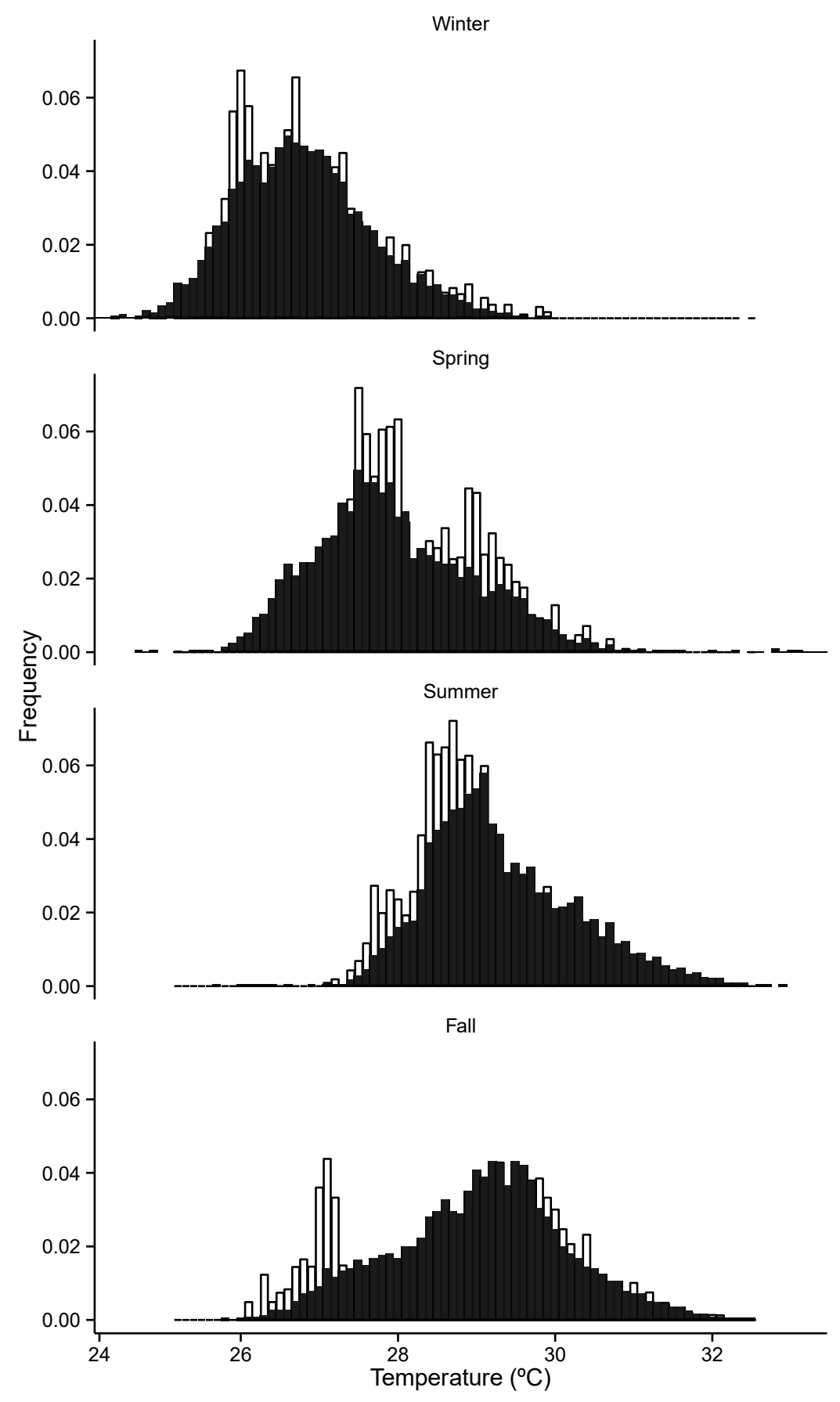


Table 3.1: Linear mixed effects model outputs for bonefish acceleration (measured with a V13AP transmitter) and metabolism $\left(\mathrm{MO}_{2}\right)$ measured in a swim tunnel respirometer

\begin{tabular}{llllll}
\hline $\begin{array}{l}\text { Response variable } \\
\text { Factor }\end{array}$ & Coefficient & SE & DF & t-value & p-value \\
\hline Acceleration & & & & & \\
Intercept & 0.57 & 0.03 & 260 & 22.78 & $<0.001$ \\
Swim speed & 0.01 & 0.00 & 260 & 43.06 & $<0.001$ \\
$\mathrm{MO}_{2}$ & & & & & \\
Intercept & 1.16 & 0.72 & 231 & 1.61 & 0.11 \\
Swim speed & 0.03 & 0.01 & 231 & 3.00 & 0.003 \\
Fork length & -0.02 & 0.01 & 63 & -1.62 & 0.11 \\
Temperature & 0.05 & 0.02 & 63 & 3.09 & 0.003 \\
Speed*Temperature & 0.0005 & 0.00 & 231 & -1.32 & 0.19 \\
\hline
\end{tabular}


Table 3.2: Significant predictors of bonefish energy expenditure $\left(\mathrm{J} \mathrm{min}^{-1} \mathrm{~kg}^{-1}\right)$ derived from accelerometer transmitters within a fine scale positioning system on Las Pelas reef flat, comparing degrees of freedom (df), Aikaikes information criterion (AIC), log-ratio test (LRT), and $p$ value based on the chi-square distribution ( $p$-value) between full and reduced models

\begin{tabular}{lcccc}
\hline & df & AIC & LRT & p-value \\
\hline None & & 10395 & & \\
Fork length & 1 & 10403 & 6.71 & 0.01 \\
Habitat:Diel & 3 & 10766 & 386.89 & $<0.001$ \\
Habitat:Season & 9 & 10859 & 452.94 & $<0.001$ \\
Habitat:Tide state & 9 & 10556 & 170.09 & $<0.001$ \\
Diel:Tide state & 3 & 10435 & 53.00 & $<0.001$ \\
\hline
\end{tabular}




\subsection{Discussion}

The field of ecological energetics explores how ecological factors such as temperature, habitat, and prey distribution influence the energetics of animals, with implications for their spatial distributions, population dynamics, and responses to anthropogenic stressors (Tomlinson et al. 2014). However, to date, few studies have examined how ecological factors influence the energy dynamics in fully aquatic species such as fish. Here I examined spatially explicit energy expenditure in a tropical marine fish, revealing the interactive effects of landscape features and temporal factors. Habitat was the strongest predictor of bonefish energy expenditure, increasing from the lowest in the deep-water lagoon, to the highest in the shallow water reef crest. Further, energy expenditure was driven mainly by variability in activity more than water temperature. Shallow, nearshore habitats are believed to represent important foraging grounds for bonefish (Colton \& Alevizon, 1983a,b; Humston et al. 2005; Murchie et al. 2013), although this has not been quantified directly in scientific studies. Animals often expend greater amounts of energy foraging, although this depends on foraging mode (Huey and Pianka 1980; Anderson \& Karasov 1981; Arnould et al. 1996). Bonefish forage actively in the benthos, searching for and capturing diverse prey such as crabs, shrimp, and small fish (Colton \& Alevizon 1983; Crabtree et al. 1998). Greater bonefish energy expenditure observed here in shallow water environments likely reflects foraging behaviour, supporting previously anecdotal evidence of foraging locations, which in Culebra were primarily the seagrass and reef crest (coral rubble) habitats. 
Landscape characteristics can have a profound effect on the energetic costs of movement (i.e., the energy landscape; Wilson et al. 2012; Shepard et al. 2013), which influences animal movement paths. Indeed, variability in water flow in river systems influenced the movement activity pallid sturgeon Scaphirhynchus albus (McElory et al. 2012), and also incurs energetic costs to Pacific salmon (Oncorhynchus spp.; Hinch \& Rand 1998; Burnett et al. 2014). Rather than examine movement paths, I explored how bonefish expend energy in relation to ecological factors including habitat type, diel period, and tidal state. While nearshore marine environments generally have less water flow than river systems, bonefish energy expenditure was highest during flooding and ebbing tides, which may be related to increased costs of movement in flowing water. Given that habitat has a significant impact on animal behavior, which is paid for in energy (Tytler \& Calow 1985), it is important to consider animal behavior and habitat function in quantifying spatially explicit energy dynamics. In this context, ecological factors such as the presence of predators or prey distribution may also generate significant variability in energetics across space and time (Lima 1986; Milinski 1986; Houston et al. 1993; Hall \& Clark 2016). By understanding the factors that contribute to animal energetics in the wild, we may gain insight into individual and population level characteristics such as growth. For example, energy dynamics may help explain why bonefish growth rates are significantly higher in Puerto Rico and Florida than The Bahamas (Crabtree et al. 1996; Adams et al. 2008).

Water temperature is a key driver of fish metabolism (Fry \& Hart 1948; Brett 1964; Clarke \& Johnston 1999), and also varied significantly across seasons in the study 
region; however, bonefish energy expenditure was relatively consistent at this time scale. Bonefish utilized this near-shore reef flat selectively at more moderate temperatures, avoiding the extreme lows in the winter and highs in the summer, moderating energy expenditure. Further, bonefish activity rates (acceleration) were also related to temperature, where activity peaked at $30^{\circ} \mathrm{C}$ and declined sharply at higher temperatures. This is consistent with the findings of Nowell et al. (2015), which showed that critical swimming speed and scope for activity decline above $30^{\circ} \mathrm{C}$ in this species. While bonefish locations were unknown when outside the range of the tracking system, bonefish in this environment have extremely high fidelity to certain reef flats (AJ Danylchuk, unpublished data), and were likely located in adjacent deeper water habitats where they frequent (Murchie et al. 2013). Water temperature is a key factor that drives habitat selection in fish (Freitas et al. 2015) as they balance tradeoffs between energy acquisition, gain, and predation risk. Because near shore habitats are important foraging grounds for bonefish, exclusion from these areas at warm temperatures may have impacts on energetics during warmer months. Under climate change scenarios, increases in water temperature may cause greater exclusion from potentially important shallow, near shore foraging grounds (Wenger et al. 2011). Indeed, energy is an important mechanism for predicting the effects of climate change on individuals and populations (Tomlinson et al. 2014).

Despite the important insights gained from quantifying energy expenditure in the wild, along with the fact that bioenergetics models are the most widely developed and applied in teleost fishes (e.g. Hansen et al. 1993; Hartman \& Brandt 1995), this study is 
one of the first to explore how fish expend energy in relation to aquatic landscape characteristics, integrating both the costs of activity and the influence of water temperature. This is likely due to the challenges of quantifying energy expenditure and position simultaneously in fully aquatic species. Global Positioning Systems do not function underwater; instead we must rely on acoustic telemetry systems to quantify position, which typically provides a very course estimate of location, within $\sim 50-1000 \mathrm{~m}$ of an acoustic receiver in three dimensions, while receivers rarely cover the entire area a fish uses, providing non-continuous data (Cooke et al. 2004). However, with recent advances in hyperbolic positioning, we can now estimate fine scale fish positions (Espinoza et al. 2011). Further, tools for estimating energy expenditure (e.g. accelerometers or heart rate loggers calibrated to estimate oxygen consumption) are typically biologgers, meaning we must rely on recapture of the tagged animal to acquire the information (Clark et al. 2010a; Gleiss et al. 2010; Brown et al. 2013), which is challenging for fish. To overcome this challenge, Payne et al. (2011) used a swim tunnel to calibrate accelerometer transmitters to estimate energy expenditure in Australian cuttlefish (Sepia apama), and estimated their metabolic rates in the field with acoustic receivers. Building on this approach, I expanded this calibration method to include the range of temperatures bonefish would experience in the wild, and tracked accelerometer tagged bonefish using a fine scale acoustic telemetry system. In doing so I was able to generate spatially explicit, activity- and temperature-based estimates of bonefish energy expenditure over an 11-month period in the wild. One major limitation with this approach is that swim tunnels generate linear swimming only; the energetic costs of more complex 
maneuvers are not incorporated despite the fact that turning can be energetically costly (Boisclair \& Leggett 1989). Accelerometer biologgers have shown promise for identifying more complex behaviors, including foraging in this species using tail beat activity and body posture data (Chapter 2), which can now be integrated into transmitter technology (de Almeida et al. 2013). Another general limitation with accelerometers is that they measure activity and behavior, and cannot capture other sources of energy expenditure such as the costs of digestion, which heart rate loggers can measure (Clark et al. 2010a). As transmitter technology develops to incorporate more sensors, future studies may integrate more accurate measures of energy expenditure, as well as energy gain via foraging.

Here I found energy expenditure is highly associated with landscape features in a teleost fish in a nearshore tropical marine system, which was mainly due to habitatspecific fish behavior. While water temperature is a major factor affecting fish metabolism, bonefish moderated energy expenditure by avoiding nearshore areas during temperature extremes. Understanding how ecological factors influence fish energy expenditure may lead to greater insight into how they distribute themselves in space and time (Shepard et al. 2013), as well as the develop of more accurate bioenergetics models (Brodie et al. 2016). Further, quantifying ecological energetics may provide insight into individual and population level dynamics such as growth rates and population sizes. This may be particularly useful for predicting how changing environmental constraints due to anthropogenic stressors such as climate change impact individuals and populations (Tomlinson et al. 2014). Aquatic ecosystem characteristics (e.g. temperature, current, 
dissolved oxygen) are extremely spatiotemporally variable, and associated energetic costs are a probable mechanism for the diversity in spatial ecology and population dynamics observed in fish. As biotelemetry and bio-logging technology continues to advance (Hussey et al. 2015), future research may aim to explore ecological energetics in fish over greater temporal and spatial scales and associations with diverse biotic and abiotic factors to gain insight in their ecology. 


\section{Chapter 4. Ecological energetics of a marine teleost fish across ecogeographic regions}

\subsection{Abstract}

Variation in ecological conditions can have significant effects on animal behaviour and bioenergetics, resulting in diverse animal life history characteristics across ecogeographic regions. Here I examine the energy expenditure of bonefish (Albula vulpes) in expansive sand flats and tidal creeks in subtropical Eleuthera, The Bahamas where bonefish growth rates are relatively slow, as well as small, fringing coral reefs in tropical Culebra, Puerto Rico where bonefish growth rates are higher. Using established relationships between bonefish acceleration, swimming speed, and oxygen consumption, I employed acoustic accelerometer transmitters and fixed station acoustic receiver arrays to estimate spatially explicit energy expenditure of free-swimming fish in both locations for over 6 months during the winter and spring seasons. Bonefish expended significantly more energy in Culebra than Eleuthera due to a combination of higher activity levels and water temperatures. However, bonefish in Eleuthera had significantly larger home ranges, moving frequently across expansive sand flats to multiple tidal creeks, while bonefish in Culebra had restricted home ranges, with high affinity to a single fringing reef. Larger home ranges undoubtedly have significant energetic costs not captured by our estimates from acceleration transmitters. These observed patterns in space use, energetics, and growth rates could be explained by the pace of life hypothesis, or may be related to the distribution of resources in the two locations, where patchier, higher density prey on 
fringing reefs in Culebra enables less energetically costly long range movements than in the expansive sand flats of Eleuthera. The distribution of resources is a potentially significant consideration for ecological energetics, with implications for animal life history traits.

\subsection{Introduction}

Energy is the currency of life for all levels of ecological organization, from individual to population, to ecosystem (Brown et al. 2004). In the wild, animals must acquire energy from the environment (via foraging) at a greater rate than energy is expended (via activity, metabolic maintenance, and digestion) to allocate resources to fitness enhancing activities, somatic growth, and reproduction (Kleiber 1961; Tytler \& Calow 1985). Environmental factors and anthropogenic stressors place energetic constraints on animals, which limit individual and population level distributions, growth rates and persistence (Fort et al. 2009; Tomlinson et al. 2014). Therefore, energy dynamics may explain animal distributions, abundances, and life history characteristics, as well as enhance our ability to quantify or predict the impacts of anthropogenic change on their populations.

The metabolic theory of ecology (Brown et al. 2004) posits that metabolic rate, as a function of body mass and body temperature, explains diverse ecological phenomena, scaling up from variation in individual traits such as growth (West et al. 2001), to population-level, and entire ecosystem dynamics (Brown \& Gillooly 2003). While this theory has much support, there are certainly some discrepancies (O'Connor et al. 2007; 
Irlich et al. 2009), likely due to the complexities of animal physiology and the influences of a multitude of ecological factors on animal energetics (Anderson \& Jetz 2005). On a species level, other potential drivers of field metabolic rates include life stage, diet, activity, habitat, and ecosystem productivity (e.g., Nagy 1987; Bryant 1997; Nagy et al. 1999). Variations in environmental characteristics across the landscape have a strong impact on animal energetics (Tomlinson et al. 2014; Ellis et al. 2012). For example, the distribution of resources and predation risk both influence the ability of animals to acquire resources via foraging (Lemon 1991; Lima 1986; Milinksi 1986). Landscape characteristics often have significant effects on the energetic costs of movement (Wall et al. 2006; Wilson et al. 2012; McElroy et al. 2012). For these reasons, energy dynamics not only influence animal distributions, but can also drive differences in animal characteristics (e.g., growth rate, life span) often observed between habitats and ecogeographic regions (Tomlinson et al. 2014; Violle et al. 2014; Chown \& Gaston 2016).

Bonefish (Albula spp.) are a group of at least 12 fish species that occupy shallow tropical and sub-tropical seas worldwide (Alexander 1961; Wallace 2014). Albula vulpes is widespread in shallow nearshore habitats throughout the Caribbean and Western Atlantic Oceans, occupying diverse habitats such as sand flats and tidal creeks in The Bahamas, fringing reefs in Puerto Rico, and seagrass flats in Florida (Humston et al. 2005, Murchie et al. 2013; Brownscombe et al. 2015). There is also corresponding variation in life history traits between ecogeographic regions; for example, growth rates of bonefish in the Bahamian archipelago are significantly lower than in Puerto Rico and Florida (Crabtree et al. 1996; Adams et al. 2008). Evidence from molecular genetics studies 
indicates there is a high level of genetic connectivity in this species between these regions in the Caribbean Sea and western Atlantic (Wallace 2014). Therefore, the observed differences in life history traits between locations are most likely due to contemporary differences in local conditions.

Environmental characteristics vary across the landscape at many spatial scales, which should have a significant influence on animal energetics (Shepard et al. 2013; Tomlinson et al. 2014). However, the energetic costs associated with occupying different habitats or ecogeographic locations are rarely quantified, particularly with fish. Here I explore the ecological energetics of bonefish, which represent a good model for this research because bonefish occupy a range of ecotypes with associated variance in life history traits. My objective was to quantify spatially explicit energy expenditure in bonefish in expansive sand flats and tidal creeks in sub-tropical Eleuthera, The Bahamas, as well as small, isolated fringing coral reefs in tropical Culebra, Puerto Rico. To accomplish this, I used accelerometer transmitters that have been calibrated to estimate energy expenditure in this species, and passive fixed station acoustic telemetry receivers to quantify energy expenditure and home range sizes in both locations. I aimed to provide some of the first insights into the influence of ecogeographic variation on the energetics of wild fish, which may help explain observed differences in life history traits in bonefish across their range. 


\subsection{Methods}

\subsubsection{Acceleration transmitter calibration}

Bonefish energy expenditure was estimated in the wild using acceleration acoustic transmitters (V9AP and V13AP, Vemco Inc., Shad Bay, NS), which measure acceleration $\left(\mathrm{g}, 9.8 \mathrm{~ms}^{-2}\right)$ in three axes ( $\mathrm{x}=$ lateral, $\mathrm{y}=$ forward, and $\mathrm{z}=$ vertical $)$. In these transmitters, measured acceleration is used to calculate the root mean square (RMS), described in Equation 1, where A represents acceleration in each axis:

1. $\mathrm{RMS}=\sqrt{A_{x}^{2}+A_{y}^{2}+A_{z}^{2}}$

The mean RMS is calculated for the sampling period ( 25 seconds) and stored on board prior to transmission. Accelerometers were surgically implanted in the coelomic cavity of bonefish using the method outlined in Murchie et al. (2011a).

Accelerometers were calibrated to predict the energy expenditure of bonefish in a two-step process. Firstly, acceleration was calibrated with bonefish $(\mathrm{n}=10 ; 42 \pm 6 \mathrm{~cm}$ fork length; mean $\pm \mathrm{SD}$; 32-53 cm range) swimming speeds using a Blazka-style swim tunnel to measure acceleration at a range of set swimming speeds (see Chapter 3 ). The relationship between acceleration (ACC) and swimming speed (SS) is described by Equation 2:

2. $\mathrm{SS}=(\sqrt{\mathrm{ACC}-0.57) / 0.013}$ 
The relationship between bonefish swimming speed and metabolic rate was developed using a Blazka-style swim tunnel respirometer at a range of water temperatures, $22-36^{\circ} \mathrm{C}$ (see Nowell et al. 2015). Briefly, untagged bonefish $(n=66,41 \pm 4$ cm fork length; mean $\pm \mathrm{SD}$; $34-51 \mathrm{~cm}$ range) were subjected to a ramp- $\mathrm{U}_{\text {crit }}$ procedure where fish swam at set speeds in $15 \mathrm{~cm} /$ second increments until exhaustion. Water dissolved oxygen concentrations were measured over a 10-minute period at each speed using an OxyGuard oxygen probe (OxyGuard Handy Polaris 2, portable DO meter, Water Management Technologies, Inc. Baton Rouge, LA, USA). The relationship between swimming speed (SS), water temperature (T) and oxygen consumption $\left(\mathrm{MO}_{2} ; \mathrm{mg} \mathrm{O} \mathrm{O}^{2}\right.$ $\left.\min ^{-1} \mathrm{~kg}^{-1}\right)$ is described by Equation 3:

3. $\sqrt{\mathrm{MO}} 2=1.16+0.03 * \mathrm{SS}-0.02 * \mathrm{FL}+0.05 * \mathrm{~T}+0.0005 * \mathrm{SS}: \mathrm{T}$

\subsubsection{Field estimates of spatial energetics}

To estimate bonefish energy expenditure in the wild in both Culebra and Eleuthera, bonefish were tagged with accelerometer transmitters using the same tagging methods as the calibration experiment, released into the wild and tracked with fixed station acoustic receivers (VR2 and VR2W receivers, 69 kHz, Vemco Inc., Shad Bay, NS). In Eleuthera, acceleration transmitters (V9AP, $6.1 \mathrm{~g}, 45-135 \mathrm{~s}$ transmission delay, \pm 3.43 acceleration range, 160 day battery life, $5 \mathrm{~Hz}$ sampling frequency) were implanted in bonefish $(\mathrm{n}=10$; $46 \pm 4 \mathrm{~cm}$ fork length; mean $\pm \mathrm{SD} ; 41-51 \mathrm{~cm}$ range) on 8-Dec-2008 (see Danylchuk et al. 2011; Murchie et al. 2011a). Bonefish were tracked with 13 acoustic receivers located 
throughout coastal habitats, including sand flats, tidal creeks, and nearshore areas up until June-2009 (104 \pm 79 days per individual, 2-174 day range). During the tracking period water temperatures were sampled once per hour at a subset of receiver locations in each habitat using Hobo-H8 temperature loggers (Onset Computer Corp, Onset MA, $\pm 0.7^{\circ} \mathrm{C}$ accuracy, $-20^{\circ} \mathrm{C}$ to $70^{\circ} \mathrm{C}$ range).

In Culebra, bonefish ( $\mathrm{n}=10,52 \pm 6 \mathrm{~cm}$ fork length; mean $\pm \mathrm{SD}$; 41-60 $\mathrm{cm}$ range) were captured via recreational angling from 27-Apr-2013 to 22-May-2013 and implanted with acceleration transmitters (V13AP, $12.2 \mathrm{~g}, 45-135 \mathrm{~s}$ transmission delay, \pm 3.43 acceleration range, 323 day battery life, $5 \mathrm{~Hz}$ sampling frequency). Tagged bonefish were tracked with an array of 59 VR2W receivers located throughout coastal habitats of Culebra, including the fore reef, back reef, lagoon, and nearshore habitats. However, 25 receivers were arranged close in proximity, comprising a fine scale positioning system. The results of fine scale spatial energetics of bonefish are reported in Chapter 3. To remove replicate detections within this system, two receivers representing fore reef and back reef habitats were selected for inclusion in this study. Bonefish were tracked up until April-2014 (218 \pm 82 days per individual, 54-332 day range). Water temperature was sampled once per hour at select receiver locations in each habitat type using temperature loggers (Hobo Pendant UA-002-64, Onset Computer Corp). 


\subsubsection{Data analysis}

A total of 22,648 detections in Eleuthera and 136,023 in Culebra were collected with acceleration values from tagged bonefish. The initial 24 hours was removed for each fish to exclude initial post-release behaviour. In Eleuthera, data from 2 bonefish were excluded due to tag malfunction. With retained data, bonefish metabolic rate was predicted from acceleration values using Equations 1 and 2 above. Energy expenditure (Joules $\min ^{-1} \mathrm{~kg}^{-1}$ ) was calculated from metabolic rate based on the standard for ammoniotelic fishes, assuming $1 \mathrm{mg} \mathrm{O}_{2}$ equated to $14.1 \mathrm{~J}$ of energy expended (Elliot \& Davidson 1975).

To compare bonefish energy expenditure between locations, the data sets were filtered to include only the Julian days where data were available from both locations, resulting in 21,216 data points in Eleuthera and 30,277 in Culebra. With this data set, a linear mixed effects model (LME) was applied with bonefish acceleration (activity) as the response variable, location (Eleuthera, Culebra) month, and location*month interaction as predictors, and fish ID as a random effect. Acceleration was log transformed prior to analysis to improve model fit. Similarly, log transformed bonefish energy expenditure (Joules $\min ^{-1} \mathrm{~kg}^{-1}$ ) was model with an LME with location, month, location*month interaction, and fish fork length as predictors and fish ID as a random effect. Fish fork length was included in the energy expenditure model in order to standardize model predictions by fish size for comparison between locations. Within each location, energy expenditure was also modeled with LMEs with habitat, diel period, and tide state as 
predictors and fish ID as a random effect. Final model fixed effects terms were determined by backward model selection using log ratio tests. Models were validated following the procedures outlined in Zuur et al. (2009).

Home ranges for each individual bonefish were calculated in both locations using the minimum convex polygon method using adehabitatHR (Calenge 2011). Home range sizes were compared between Eleuthera and Culebra using a one-way ANOVA. Home range size was log transformed prior to analysis to satisfy assumptions of normality and homogeneity. Statistical analyses were conducted using RStudio version 0.99.491 (R Core Team 2013) and the level of significance was considered at $\mathrm{p}=0.05$. All data are presented as mean \pm standard deviation unless otherwise specified.

\subsection{Results}

\subsubsection{Activity, temperature, and energy expenditure}

Bonefish activity levels were generally higher in Culebra than in Eleuthera in the winter months, but were similar between locations in the spring season (Fig. 4.1A). Overall, activity levels were not significantly different between locations (LME; $F_{1,16}=2.6$, $\mathrm{p}=0.13$ ). However there was a significant interaction between location and month in predicting activity $\left(\mathrm{LME} ; \mathrm{F}_{5,51770}=68.5, \mathrm{p}<0.001\right)$, which reflected seasonal patterns. Water temperatures associated with bonefish positions were consistently higher in tropical Culebra than sub-tropical Eleuthera in all months (Fig. 4.1B). Due to a 
combination of higher activity levels and water temperatures, bonefish energy expenditure was higher in Culebra than Eleuthera, particularly in the winter months (Fig. 4.1C). Location, month, their interaction, and bonefish fork length (FL) were all significant predictors of bonefish energy expenditure (Table 4.1). The average sized bonefish in this study (49 cm FL) was predicted to expend $47.6 \pm 7.9$ Joules $\mathrm{min}^{-1} \mathrm{~kg}^{-1}$ in Eleuthera, compared to $62.5 \pm 4.2$ Joules $\mathrm{min}^{-1} \mathrm{~kg}^{-1}$ in Culebra, which is $31 \%$ higher (Fig. 4.1C).

\subsubsection{Spatiotemporal factors}

Bonefish energy expenditure varied significantly across temporal factors, diel period and tide state, as well as between habitats in both Eleuthera and Culebra (Fig. 4. 2; Table 4.1). Energy expenditure was greater during the day than at night in both locations, with a more drastic difference in Eleuthera (Fig. 4.2A). Energy expenditure was consistently highest during low and ebbing tides in both locations (Fig. 4.2B). Examining spatial patterns, bonefish in Eleuthera tended to expend more energy on nearshore flats, followed by creek mouths and nearshore habitats, expending the least energy in tidal creeks (Fig. 4.2C). In Culebra, energy expenditure was highest in nearshore and fore reef habitats, followed by lagoon and back reef habitats. There was a general pattern in both locations of increased energy expenditure in habitats with deeper water and greater distances from shore. 


\subsubsection{Home ranges}

The acoustic receiver array in Culebra covered a more expansive spatial area with a greater number of receivers, but bonefish in Eleuthera were detected at more receivers (10 \pm 1 receivers/individual) than Culebra ( $7 \pm 1$ receivers/individual), spanning a larger area (Fig. 4.2). Calculation of minimum convex polygons indicated bonefish had significantly larger home range sizes in Eleuthera $\left(6.4 \pm 2.3 \mathrm{~km}^{2}\right)$ than Culebra $(0.2 \pm 0.1$ $\mathrm{km}^{2}$; one-way ANOVA; $\left.\mathrm{F}_{1,13}=19.4, \mathrm{p}<0.001\right)$. While bonefish in Culebra were typically detected only in proximity to one small, isolated reef flat, bonefish in Eleuthera moved frequently between nearshore areas, including tidal creeks and nearshore sand flats. 
Fig. 4.1: A) Bonefish activity (acceleration) across months of the year in Culebra (black) and Eleuthera (grey), B) associated environmental temperatures, C) predicted bonefish energy expenditure (Joules $\mathrm{min}^{-1} \mathrm{~kg}^{-1}$ ) from a linear mixed effects model.

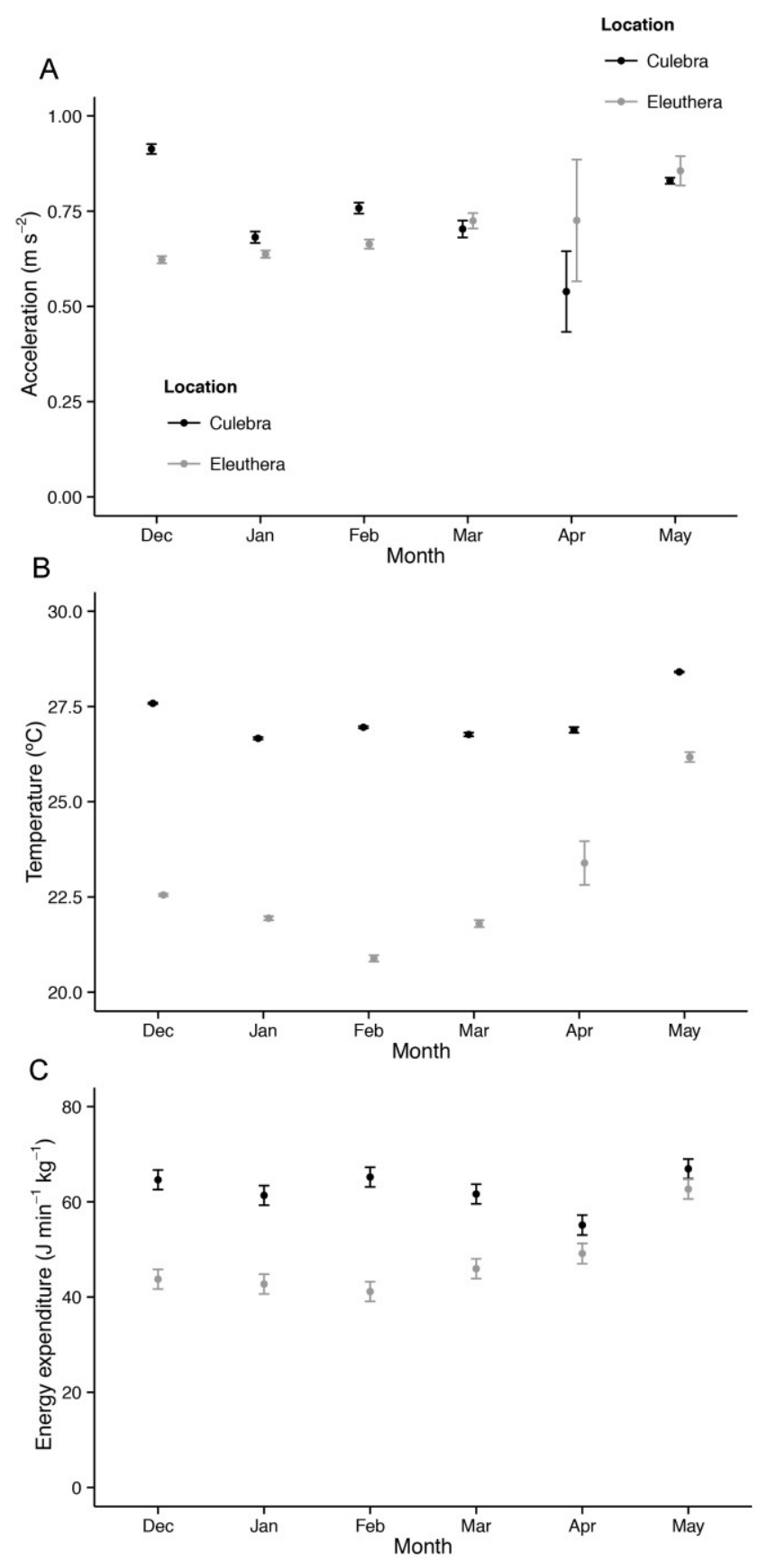


Fig. 4.2: Bonefish energy expenditure (Joules $\mathrm{min}^{-1} \mathrm{~kg}^{-1}$ ) in Culebra (black) and Eleuthera (grey) across A) diel periods, B) tide states, C) habitats. Error bars represent $95 \%$ confidence intervals.

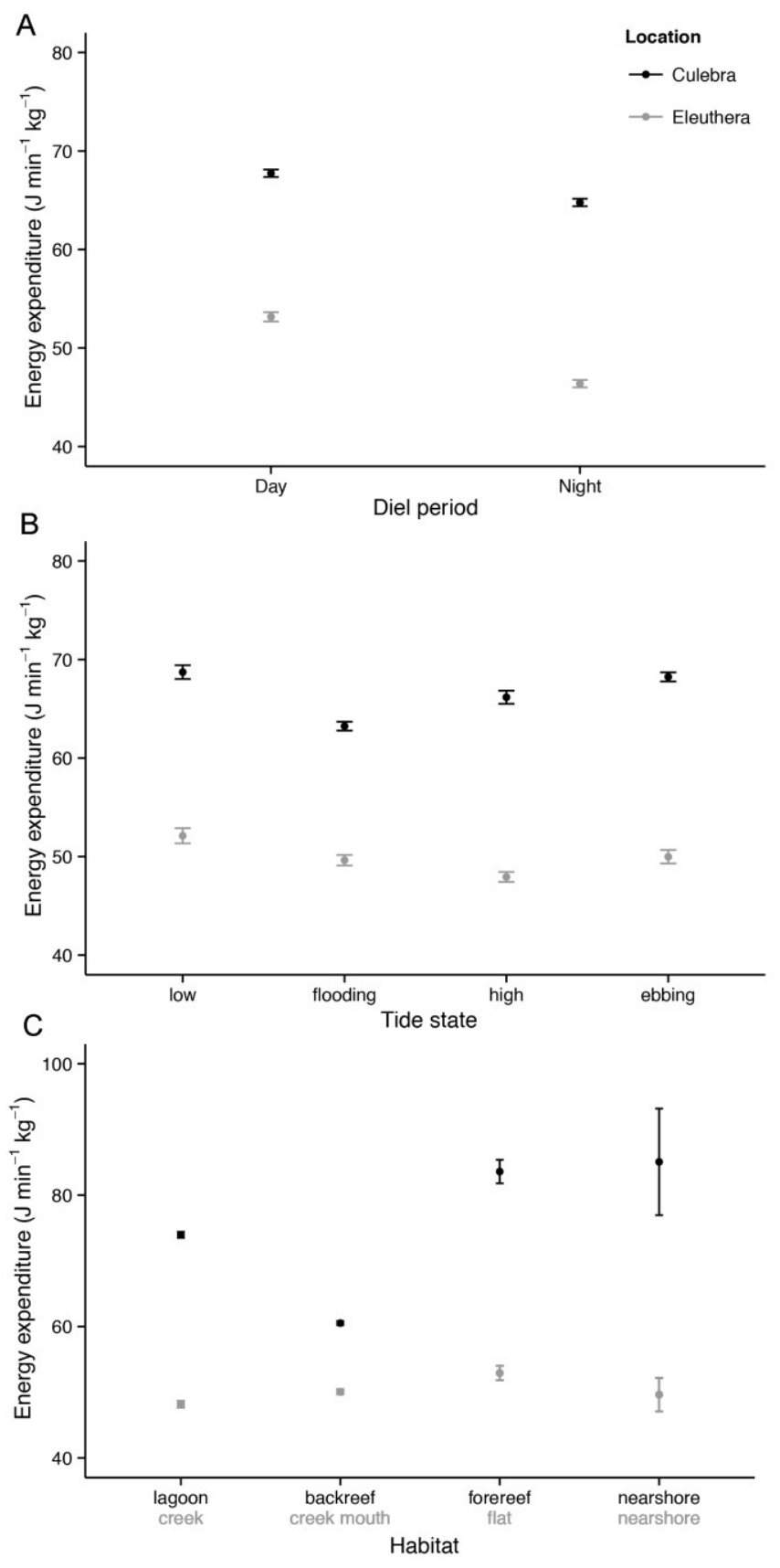


Fig. 4.3: Home ranges (95\% minimum convex hulls; green) of bonefish with the median home range size in A) Eleuthera, and B) Culebra. Yellow circles represent acoustic receiver locations; maps are equivalent in scale.

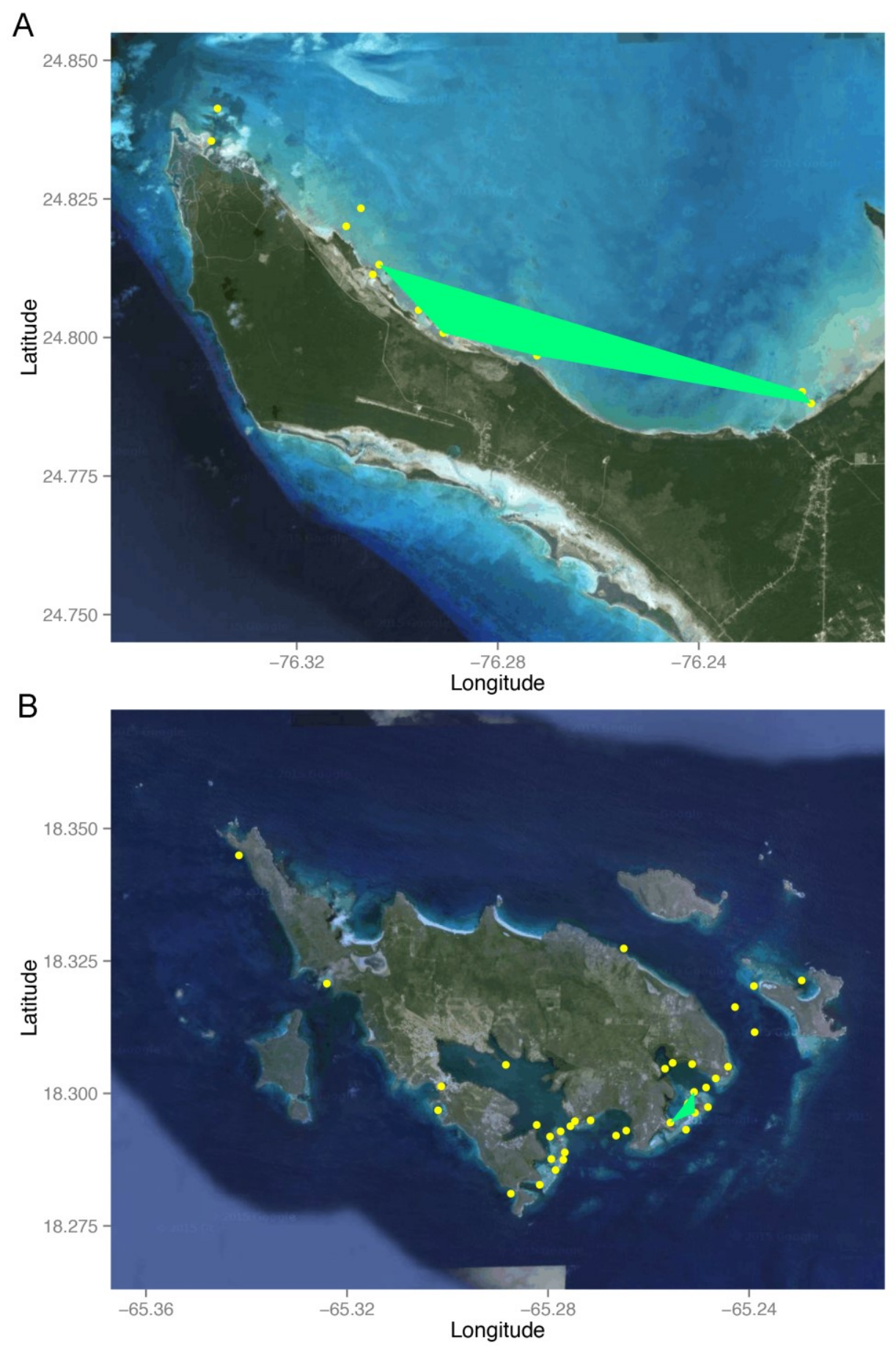


Table 4.1: Linear mixed effects model outputs for bonefish energy expenditure log(Joules min-1 kg-1) with both locations (Combined), predictors including location, month, bonefish fork length, and location*month interaction as predictors, as well as in each location separately (Culebra, Eleuthera) with habitat, diel period, and tide state as predictors. For all models fish ID was included as a random effect.

\begin{tabular}{|c|c|c|c|c|}
\hline Model & numDF & denDF & F-value & p-value \\
\hline \multicolumn{5}{|l|}{ Predictor } \\
\hline \multicolumn{5}{|l|}{ Combined } \\
\hline Location & 1 & 15 & 11 & 0.005 \\
\hline Month & 5 & 51770 & 140 & $<0.0001$ \\
\hline FL & 1 & 15 & 13 & 0.002 \\
\hline Location*Month & 5 & 51770 & 165 & $<0.0001$ \\
\hline \multicolumn{5}{|l|}{ Culebra } \\
\hline Habitat & 3 & 30261 & 530 & $<0.001$ \\
\hline Diel period & 3 & 30261 & 84 & $<0.001$ \\
\hline Tide state & 1 & 30261 & 196 & $<0.001$ \\
\hline \multicolumn{5}{|l|}{ Eleuthera } \\
\hline Habitat & 3 & 21201 & 63 & $<0.001$ \\
\hline Diel period & 3 & 21201 & 40 & $<0.001$ \\
\hline Tide state & 1 & 21201 & 625 & $<0.001$ \\
\hline
\end{tabular}




\subsection{Discussion}

Variation in environmental characteristics can have a significant impact on the energy dynamics of wild animals, which in turn may influence individual- and population-level characteristics, with implications for community and whole ecosystem processes (Brown et al. 2004; Tomlinson et al. 2014). Here I explored the energy expenditure of bonefish in two very different ecogeographic regions, expansive sand flats and tidal creeks of Eleuthera, The Bahamas, and small, isolated fringing reefs of Culebra, Puerto Rico during the winter and spring seasons. Overall, acceleration- and temperature-based estimates of bonefish energy expenditure were higher in Culebra than Eleuthera $(+31 \%$ controlling for fish size), particularly in the winter months due to a combination of greater fish activity levels and higher water temperatures. For ectotherms, temperature is considered the most significant environmental diver of energy expenditure (Fry \& Hart 1948; Brett 1964; Clarke \& Johnston 1999), and also affects aerobic scope for activity (Farrell et al. 2008). Maximum aerobic scope for bonefish occurs at $27^{\circ} \mathrm{C}$, and sharply declines above $30^{\circ} \mathrm{C}$ (Nowell et al. 2015). Differences in temperature may explain the greater activity rates observed in bonefish in tropical Culebra than subtropical Eleuthera during the winter and spring seasons.

Within regions, bonefish also exhibited significant variation in energy expenditure across spatiotemporal variables, with some interesting consistencies between the two locations. While the habitats are very different between locations, bonefish in both Eleuthera and Culebra tended to expend a less energy in shallow, near shore habitats (i.e., 
tidal creeks in Eleuthera and back reefs in Culebra). Bonefish expended energy at greater rates on the ocean side of their habitats, at the tidal creek mouths and coastal sand flats in Eleuthera, and fore reefs of Culebra. Landscape features are often highly influential on animal energy expenditure due to the costs of movement and habitat specific behaviour (Wilson et al. 2012; Shepard et al. 2013). Given that water temperatures were generally higher in nearshore habitats, activity levels drove the observed pattern of increased energy expenditure in deeper water habitats. This likely reflects the behavioural ecology of bonefish, which migrate into shallow nearshore areas with the tides to forage (Murchie et al. 2013). Indeed, bonefish energy expenditure was highest during outgoing and low tides, when they are typically transitioning into deeper water habitats.

While estimates of energy expenditure were significantly lower in Eleuthera than Culebra, bonefish home range sizes were significantly larger in Eleuthera, including expansive sand flats and multiple tidal creeks, while in Culebra bonefish remained within the vicinity of one isolated fringing reef. Generally, animal home ranges tend to increase with body size as well as other factors including metabolic rate (energetic requirements), population density, resource availability and distribution, and neighbor intrusion (McNab 1963; Jetz et al. 2004; Mace \& Harvey 1983). Home range size is also often associated with prey density, where lower densities necessitate larger home ranges (Letourneur, 2000). Bonefish sizes were generally larger in Culebra than Eleuthera, but the fish had significantly smaller home ranges. This was likely due to prey distribution, where isolated fringing reefs represent small patches of high-density prey, with relatively large gaps in between reefs. Movement between reefs through deeper water in Culebra also 
likely incurs greater predation risk (Sih 1984; Rypel et al. 2007). Further, the tidal fluxes are much greater in Eleuthera (mean tidal flux $0.75 \mathrm{~m})$ than Culebra $(0.22 \mathrm{~m})$, and the intertidal zones in Eleuthera are therefore more expansive; necessitating greater movement distances to exploit temporally available resources and reduced predation risk in these areas (McIvor \& Odum 1988; Clark et al. 2003). In general, fish home ranges tend to be larger in coastal regions with greater tidal variations (Krumme 2009).

Bonefish in Puerto Rico exhibit significantly higher growth rates than in Eleuthera (Adams et al. 2008). Given the high level of connectivity between these demes of bonefish (Wallace 2014), observed differences are likely related to phenotypic variation due to regional conditions. Bonefish experience higher water temperatures in tropical Puerto Rico than subtropical Eleuthera, and temperature is considered the most important predictor of growth rates in fish because it limits the rate of metabolic processes (Brett 1979). In ectotherms, the pace of life tends to be higher (faster growth and maturation rates, shorter life span) at warmer temperatures (Angilletta et al. 2004). While temperature influences growth rates, bonefish must also consume a greater amount of resources to fuel growth, as well as size- and temperature-related metabolic demands. Preliminary bonefish diet studies show a high degree of similarity between the regions (Colton et al. 1983; L. Griffin, unpublished data). Although prey availability and foraging efficiency have not been quantified in these regions, coral reefs are highly productive ecosystems compared to sand flats (Pamatmat 1968; Hatcher 1990; Moberg \& Folke 1999). 
While prey abundance is a key factor in consumption and growth rates, prey distribution is also highly relevant (Lemon 1991). If prey is less concentrated there is a greater energetic cost to foraging and lower net energetic gain. In regards to space use, optimal foraging theory posits animals should remain foraging in a patch until foraging efficiency declines below a certain level, making movement to a new patch energetically favourable (Charnov 1976; Pyke 1984). Indeed, home range size is often negatively correlated with prey abundance (Litvaitis et al. 1986; Letourneur 2000). While the exact distribution of prey is unknown in these environments, the fact that bonefish traverse such large areas, frequenting a wide range of sand flats and tidal creeks in Eleuthera suggests that the density of prey is low enough to make these movements energetically favourable. Conversely, bonefish in Culebra likely take advantage of small areas of high prey abundance around fringing reefs to minimize home ranges and energetic costs. The large home range sizes of bonefish in Eleuthera compared to Culebra, related to lower densities of lower energy prey, is a potential explanation for the differences in growth rates observed in these regions. In the context of ecological energetics (Tomlinson et al. 2014) and perhaps even the energy landscape (Wilson et al. 2012; Shepard et al. 2013; Mosser et al. 2014), prey distribution may be an important consideration for the energetic costs of inhabiting various environments, with implications for individual and population level traits.

It is well established that habitat features have a strong influence on animal characteristics, including life history traits such as growth and reproduction (Hayes et al. 1994; Langerhans 2003). Despite the fact that habitat is recognized as an important factor 
influencing field metabolic rates of animals (Nagy et al. 1999), and various spatiotemporal factors influence both energy expenditure and gain in wild animals (Shepard et al. 2013; Tomlinson et al. 2014), studies focusing on this mechanistic link to animal characteristics (e.g., life history traits such as growth) are rare. Gauthier et al. (1984) examined the condition factor and energy expenditure of snow geese during reproductive staging in two different types of marsh habitats; they found that individuals had significantly higher energy stores, and expended a greater amount of energy in the freshwater marshes than salt marshes. Gauthier et al. (1984) also noted the salt marsh covered a much larger area, but did not explicitly quantify space use or home range size. These findings are similar to our observations with bonefish, where the deme with high rates of energy expenditure and small home ranges exhibit higher rates of growth. While many studies address the relationship between energetic requirements and home range size (McNab 1963), examinations of the energetic costs of having a larger home range due to the distributions of resources are less explored, and may be an important driver of animal characteristics across ecogeographic regions.

Estimates of fish bioenergetics have a rich history, but mainly in laboratory settings (e.g. Hansen et al. 1993). More recently, estimates of field metabolic rates have revealed diverse patterns of fish energy expenditure across space and time, focusing primarily on salmonids in river systems (Hinch \& Rand 1998; Wilson et al. 2013; Burnett et al. 2014). This study represents, to my knowledge, the first time fish energy expenditure has been estimated across such broad spatial and temporal scales. However, my data were derived from different years in each location, and the time periods where 
Julian days overlapped spanned only half of the year (during winter and spring). While it is uncertain how consistent this pattern would be during summer and autumn, it is intuitive that ectothermic fish expend a greater amount of energy in tropical environments than subtropical counterparts given the tight link between temperature activity, and fish metabolism (Clarke \& Johnson 1999; Halsey et al. 2015). Another limitation to our approach is it provides discontinuous data, where fish must be in range of a receiver to be detected. Therefore, while metabolic estimates were higher for bonefish in Culebra, bonefish home range sizes were significantly larger in Eleuthera, which may have considerable energetic costs that are not captured by our receiver data. Fish moving quickly (expending a high level of energy) through expansive nearshore areas are less likely to be documented using the passive receiver tracking method, and these costs are probably underrepresented here. Future studies may provide more comprehensive estimates of field metabolic rates in fishes through increased receiver coverage, or using biologging technology, as I did in a semi-natural environment in Chapter 2.

Here I found bonefish occupying small, isolated, fringing reefs in tropical Culebra expend a greater amount of energy in the winter and spring months than their counterparts in expansive sand flats and tidal creeks in subtropical Eleuthera due to a combination of higher activity levels and water temperatures. However, bonefish in Eleuthera have significantly larger home range sizes than in Culebra, and the energetic costs may be significant but missed by our approach to quantifying energetics with nodes of passive acoustic receivers. Bonefish occupying the fringing reefs of Puerto Rico may experience higher growth rates than Eleuthera due to a combination of higher resource 
availability (temperature and food), as well as more clumped resources, enabling less energetically costly movement across the landscape. Future research should aim to acquire more continuous energy expenditure data, as well as information on energy intake via foraging, prey distributions, and predation risks to help clarify relationships between ecological energetics and life history traits. 


\section{Chapter 5. Recovery bags reduce post-release impairments in locomotory activity and behaviour of bonefish following exposure to angling-related stressors}

\subsection{Abstract}

Bonefish (Albula spp.) are a group of species targeted by recreational anglers in shallow tropical and sub-tropical seas worldwide. Although bonefish angling is almost entirely catch-and-release, mortality can occur because the stress associated with angling and handling causes locomotory impairment that promotes post-release predation. I used triaxial accelerometer loggers to compare the locomotor activity and behaviour of bonefish exposed to angling-related stressors and immediately released $(\mathrm{n}=10,39.9 \pm 1.1 \mathrm{~cm} \mathrm{FL})$, to those retained in a recovery bag for 15 min prior to release $(n=10,39.6 \pm 1.0 \mathrm{~cm} F L)$ in a tidal creek in Eleuthera, The Bahamas. I also validated the use of reflex action mortality predictors (RAMP) as an impairment index for evaluating bonefish condition upon release. Following release, bonefish were visually tracked for $30 \mathrm{~min}$ with floats to evaluate short-term survival, after which the accelerometer was retrieved. Bonefish held in recovery bags exhibited significantly less locomotory impairment immediately postrelease, and higher maximum tail beat frequencies and amplitudes up to 15 minutes postrelease, which was likely due to the time spent in the recovery bag. Bonefish in the recovery bag treatment also spent more time resting in possible refuge areas, which may facilitate further recovery and avoidance from predation. RAMP provided a gradient of impairment scores that were correlated with stressor duration. Retaining bonefish in recovery bags improved swimming abilities during the critical time period where the 
majority of post-release predation occurs, and one fish that was not placed in the recovery bag was preyed upon during the monitoring period. Further testing is needed to determine if the locomotory and behavioural benefits of retaining bonefish in recovery bags translate into improved survival from predation in more predator rich environments.

\subsection{Introduction}

Catch-and-release angling $(\mathrm{C} \& \mathrm{R})$ is highly popular worldwide, practiced voluntarily due to the conservation ethic of anglers or when mandated by harvest regulations (Cowx 2002; Cooke \& Cowx 2004). It is a socially and economically important activity, as well as a conservation strategy that relies upon the assumption that released fish will ultimately survive (Wydoski 1977; Arlinghaus et al. 2007). However, C\&R angling can have detrimental effects on captured individuals, which could contribute to population level declines (Lewin et al. 2006; Cooke \& Schramm 2007). Physical injuries often result from various aspects of the angling process, including hooking, netting, and handling (Muoneke \& Childress 1994; Barthel et al. 2003; Danylchuk et al. 2008). Angling also causes physiological stress due to exhaustive physical exercise and air exposure (Cooke et al. 2002; Arlinghaus et al. 2009). These physical injuries and physiological impairments have the potential to cause post-release mortality, or even reduced growth and fitness (Cooke \& Schramm 2007). Behavioural impairments (reduced locomotory capabilities) can reduce reproductive potential or increase vulnerability to predation, while reduced fitness may result from the energy expenditure required to recover from 
physical and physiological disturbances (Cooke et al. 2000; Danylchuk et al. 2007a).

However, the extent to which behavioural impairments affect the fitness of angled fish is not well understood, particularly in marine systems where predators are prevalent (Cooke et al. 2002).

A variety of angler behaviours and gear choices influence the outcome of a C\&R event for a fish (reviewed in Cooke \& Suski 2005). The application of best angling practices, including using the appropriate gear, reducing fight time, and minimizing air exposure have been shown to reduce stress and improve survival for a variety of fish species (reviewed in Cooke \& Suski 2005; Cooke \& Schramm 2007; Arlinghaus et al. 2007). There is also growing interest in developing and testing strategies that have the potential to facilitate physiological and behavioural recovery and thus improve survival. Recovery gears can range from special devices intended to facilitate ram ventilation using water pumps (e.g., Farrell et al. 2001; Bettinger et al. 2005) to more simple gears where fish are held in static water (e.g., a cooler or portable bag). For species where post-release mortality is documented to be prevalent, use of recovery gears could be an effective strategy to enable fish to compensate for physiological and behavioural impairments and thus improve the ability of fish to evade predators. However, to our knowledge this idea has never been tested for marine fish that are subject to high levels of post-release predation.

Bonefish (Albula spp.) are a group of benthivorous fish species that occupy shallow tropical and subtropical seas worldwide (Alexander 1961), and are highly 
popular for recreational anglers, generating important revenue for many local economies (Humston 2001; Danylchuk et al. 2008). Despite the fact that angling for bonefish is almost entirely $\mathrm{C} \& \mathrm{R}$, the stress of angling causes locomotory impairment that often leads to predation (Cooke \& Philipp 2004; Danylchuk et al. 2007a). Cooke \& Philipp (2004) noted that post-release mortality of bonefish was influenced by predator abundance and could be as high as $40 \%$. Danylchuk et al. (2007a) revealed that bonefish that lost equilibrium (a reflex impairment; Davis 2010) prior to release had behavioural impairments and were six times more likely to be preyed upon than angled fish that had not lost equilibrium. These studies also found that the majority of mortality occurred in the first 20 min post-release, suggesting that this is the critical period for survival after angling events. Therefore it has been theorized that retaining bonefish for a short period may reduce mortality, especially in areas of high predator abundance (Cooke \& Philipp 2008), although this has not yet been validated as an effective practice.

The primary objective of this study was to evaluate the effectiveness of retaining bonefish in recovery bags for reducing short-term locomotory impairment when subjected to angling-related stressors, and whether potential improvements in swimming ability translated to increased survival. I used physiological stressors and did not inflict physical injuries that are often associated with angling events because previous studies have shown that it is the former that lead to behavioural impairments and post-release predation (see Cooke \& Philipp 2004; Danylchuk et al. 2007a). I used recovery bags given that they are inexpensive and could be easily carried by anglers when walking or wading rather than gears that required electricity or were otherwise less likely to be 
embraced by anglers due to practicality and expense. To quantify post-release behaviour I used novel high-resolution tri-axial accelerometer loggers, which allowed for the measurement of body acceleration in three axes to quantify tail beat frequencies and amplitudes. I also tested reflex impairment indices as indicators of bonefish condition given that previous work by Danylchuk et al. (2007a) revealed that loss of equilibrium was associated with post-release predation. Reflex indicators have recently been deemed effective predictors of mortality (Davis 2010; Raby et al. 2012), and could be used by anglers to evaluate in which instances fish would benefit from recovery. I predicted that fish retained in recovery bags would exhibit lower reflex impairment, as well as higher locomotory ability and survival than those immediately released.

\subsection{Methods}

\subsubsection{Study site and fish collection}

This study was conducted in Kemps Creek, Eleuthera, The Bahamas $\left(24^{\circ} 48.9^{\prime} \mathrm{N}, 76^{\circ}\right.$

$\left.18.1^{\prime} \mathrm{W}\right)$. Kemps is a tidal creek with mainly coarse sand substrate, and sparse vegetation, including primarily Halimeda (Halimeda spp.), and Penicillus (Penicillus spp.). The shoreline is lined with red mangrove (Rhizophora mangle), and sharp calcium carbonate rock. Preliminary genetic analysis indicated that all bonefish in this area were Albula vulpes (Danylchuk et al. 2007a). Bonefish were collected by block netting (see Danylchuk et al. 2011) in Kemps Creek on 23-Feb-2012, and were held in a mesh pen 
$(1.3 \mathrm{~m} \times 0.8 \mathrm{~m} \times 1.25 \mathrm{~m}, 3.1 \mathrm{~cm}$ extruded plastic mesh) for up to $48 \mathrm{hr}$ prior to experimentation.

\subsubsection{Validation of reflex impairment indices}

I validated the use of reflex action mortality predictors (RAMP) (Davis 2005, 2010) to assess bonefish vitality after $0,2,4$ and 6 min of air exposure. The 0 min assessments $(n=30)$ occurred prior to air exposure on fish from all treatments, while bonefish in the 2 min treatment ( $\mathrm{n}=20)$ were those used in recovery bag experiments (see below), and 4, 6 min treatments $(\mathrm{n}=5)$ were conducted on alternate fish. Five predictors were measured; tail grab, equilibrium (orientation), body flex, head complex, and vestibular-ocular response (VOR). These predictors were chosen because Raby et al. (2012) found they were strong predictors of coho salmon (Oncorhynchus kisutch) mortality after being caught in commercial nets, and all these predictors can be easily and quickly measured by bonefish anglers. RAMP was assessed in the same manner as Raby et al. (2012). The presence of a tail grab response was assessed by grabbing the fish's tail while submerged in water; it was considered impaired if the fish did not attempt to swim away from the handler. Equilibrium was assessed by rolling the fish upside down in water; impairment was indicated when the fish was unable to right itself within $3 \mathrm{sec}$. Body flex was tested by holding the fish by the middle of the body in air; it was considered impaired if the fish made no attempt to struggle free. Head complex was considered impaired if while holding fish in air, a regular pattern of ventilation of the fish's operculum was not 
observed for at least $5 \mathrm{sec}$. VOR was assessed by rolling the fish back and forth in air; it was considered impaired if its eyes did not roll to maintain the same pitch and track the angler. Higher RAMP scores indicated greater impairment.

\subsubsection{Post-release activity experiments}

Bonefish were retrieved from the holding pen by dip net and held in a trough filled with seawater for attachment of tri-axial accelerometer loggers (model X6-2, $20 \mathrm{~g}$ in air, $25 \mathrm{~Hz}$ recording frequency; model X6-2mini, $500 \mathrm{mAh}$ battery, $15 \mathrm{~g}$ in air, or $250 \mathrm{mAh}$ battery, $10 \mathrm{~g}$ in air, $20 \mathrm{~Hz}$ recording frequency; Gulf Coast Data Concepts, Waveland, MS). Triaxial accelerometers measure dynamic and static acceleration in units of gravity $(\mathrm{g})$, equivalent to $9.8 \mathrm{~m} \mathrm{~s}^{-2}$ (Wilson et al. 2008). Larger tags were used on larger individuals, and an equal number of each tag type was used for each treatment. Devices were attached externally, secured through the dorsal musculature below the dorsal fin to plastic frontal and backing plates ( $2 \mathrm{~g}$ each) with $36 \mathrm{~kg}$ strength braided Dacron line (Fig. 5.1). Tags were oriented with the y-axis facing longitudinally. A cylindrical foam float was also attached to the frontal plate with $2.5 \mathrm{~m}$ of $7 \mathrm{~kg}$ strength monofilament line for visual tracking of the fish (as per Cooke and Philipp 2004; Danylchuk et al. 2007a) and retrieval of the accelerometer loggers. The attachment procedure lasted less than one minute.

Tagged bonefish were then assessed for RAMP, and air exposed in a moistened rubber net or recovery bag (not submerged) for two minutes. This elicited physical exercise as the fish struggled in the net, as well as air exposure, which cumulatively result 
the depletion of tissue energy stores (e.g., PCr, ATP, glycogen of white muscle) and elevations of lactate in white muscle and plasma, similar to the stress of angling events (Suski et al. 2004). Our stressor duration (2 min) was similar to a relatively quick angling event for bonefish (Cooke \& Philipp 2004; Danylchuk et al. 2007a,b). However, over $90 \%$ of fish lost equilibrium post-stress in this study, indicating it was more similar to a highly stressful angling event (Cooke \& Philipp 2004; Danylchuk et al. 2007a).

RAMP was reassessed after air exposure, and bonefish were either immediately released $(n=10)$ or held in a recovery bag (Dynamic Aqua Ltd., Vancouver, BC; 75 cm length, $15 \mathrm{~cm}$ width with $0.5 \mathrm{~cm}$ mesh on both ends and a plastic zipper; see Fig. 5.2) for 15 min prior to release $(n=10)$. Immediately released fish were manually resuscitated for up to 1 min prior to release; a common practice amongst recreational anglers.

Resuscitation involved holding bonefish upright, facing tidal currents to allow water to flow through the mouth and over the gills. Swimming data was collected for only nine bonefish in the immediate release treatment because data from one bonefish was lost due to a predation event, while all ten accelerometers were recovered from fish in the recovery treatment. For bonefish held in recovery bags, RAMP was reassessed prior to release. Releases occurred at water depths of $25-70 \mathrm{~cm}$.

Tagged bonefish were visually tracked by wading, following the attached float at a distance of at least $15 \mathrm{~m}$ for 30 minutes. Accelerometers were retrieved using rod and reel to hook the float line and recapture the fish. Trials were conducted between 0700$1500 \mathrm{hr}$ on February $24^{\text {th }}$ and $0700-1000 \mathrm{hr}$ on $25-\mathrm{Feb}-2012$. Treatments were alternated 
to achieve similar environmental conditions between treatment groups. Water temperatures ranged from $24-30^{\circ} \mathrm{C}$ during this time period, which is a typical temperature range for these environments during this time of year (Murchie et al. 2011b).

\subsubsection{Data analysis}

Accelerometers were set to continuously record total acceleration (g) at intervals of 20 or $25 \mathrm{~Hz}$ in three (x, y and $\mathrm{z}$ ) planes, where total acceleration was the sum of both static (due to gravity) and dynamic (due to animal movement) acceleration with maximum values of $\pm 6 \mathrm{~g}$. Device output was calibrated by rotating the device through known angles to real $\mathrm{g}\left(9.8 \mathrm{~m} \mathrm{~s}^{-2}\right)$ prior to deployment (Gleiss et al. 2010). Static and dynamic acceleration were separated by weighted smoothing at an interval of $2 \mathrm{sec}$. The ideal smoothing interval was determined following the method of Shepard et al. (2008), and smoothing was conducted using OriginPro 8 software (OriginLab, Northampton, MA).

To quantify bonefish behaviour, as well as tail beat frequencies and amplitudes, continuous wavelet transformation was used to decompose acceleration data based on the amplitudes and frequencies of oscillations in the sway axis (following the method of Sakamoto et al. 2009). Cycles ranging in frequency from 0.1 to 1 seconds were included in analysis, and the non-dimensional frequency parameter was set to eight to best identify oscillations in sway axis acceleration for bonefish. Data were then clustered into similar spectra using the $k$-means algorithm. Spectra were categorized into a maximum of 10 clusters, as higher numbers were found to add no resolution to swimming behaviours, but 
instead separated resting behaviours (defined by low amplitude, non-cyclic movements; Whitney et al. 2010). For clusters that represented swimming behaviour (cyclic movements), tail beat frequency and amplitude were determined from the cycle frequency and amplitude of clusters (Fig. 5.3). This was not a measure of actual tail beat amplitudes, but relative amplitudes of sine waves derived from acceleration data in units of acceleration (g). Spectra were further categorized into resting (low frequency, non oscillating movements), slow ( $<2$ tail beats/sec, and $/$ or $<0.1 \mathrm{~g}$ amplitudes), moderate ( $2-3$ tail beats/sec, 0.1-0.2 g amplitudes) or fast ( $>3$ tail beats $/ \mathrm{sec},>0.2 \mathrm{~g}$ amplitudes) swimming speeds (Fig. 5.3). Behavioural analysis was conducted using Igor Pro 6.0 software (WaveMetrics Inc., Lake Oswego, OR), and Ethographer (see Sakamoto et al. 2009).

\subsubsection{Statistical analysis}

The fork length of bonefish was compared between recovery and immediate release treatments using an independent t-test. To compare locomotory activity between treatments during equivalent time periods post-release, mean and maximum tail beat frequency, as well as mean and maximum tail beat amplitude were compared using a mixed measures ANOVA with treatment as the fixed effect, and time as the repeated measure in 1-minute intervals for the first 10 minutes post-release. Due to interaction effects, these variables were compared between treatments within each minute using Bonferroni corrected independent t-tests. To compare locomotory activity between 
treatments during equivalent time periods post-stressor, the same variables were compared from 15 to 25 minutes post-stressor in 1-minute intervals using a mixed measures ANOVA. Due to interaction effects, mean tail beat amplitudes were compared within each minute using independent t-tests. All variables were tested for assumptions of normality, homogeneity of variance, and in the case of the mixed measures ANOVA, sphericity prior to analysis. Where data did not meet the assumption of sphericity, a Greenhouse-Geisser correction factor was applied (Field 2012). All statistical analyses were conducted in R (v.2.15 R Foundation for Statistical Computing, Vienna, Austria). All data are presented as mean \pm SE unless otherwise specified. 
Fig. 5.1: Location of accelerometer attachment on bonefish for quantifying behaviour after simulated angling stress.

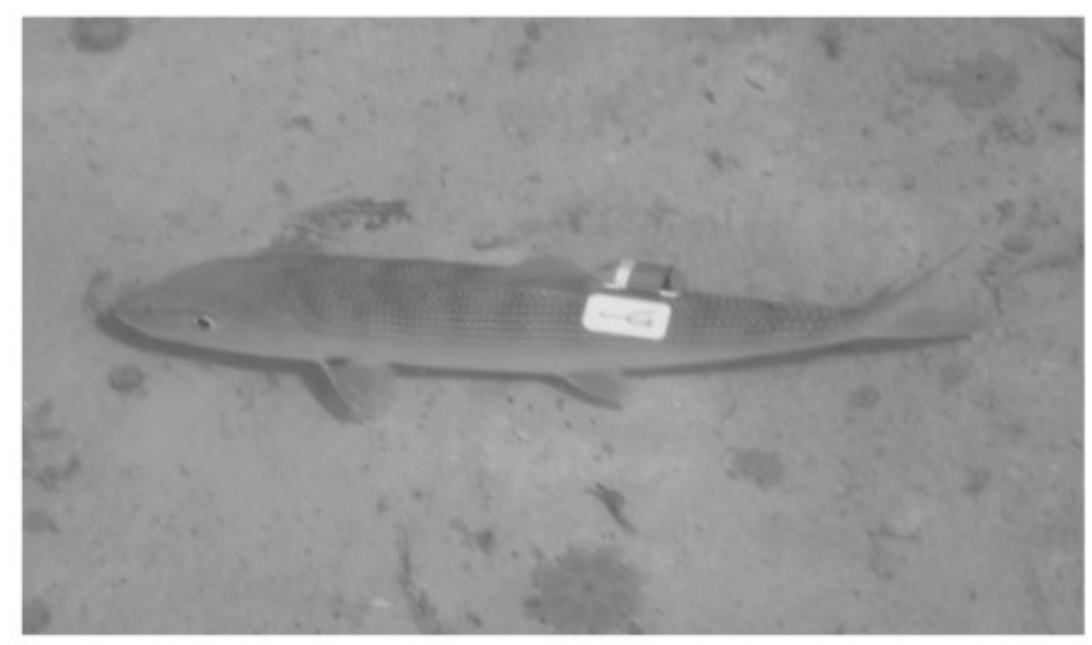


Fig. 5.2: Recovery bag being used to retain bonefish after angling by wading, behind a boat, and fastened to a mangrove.
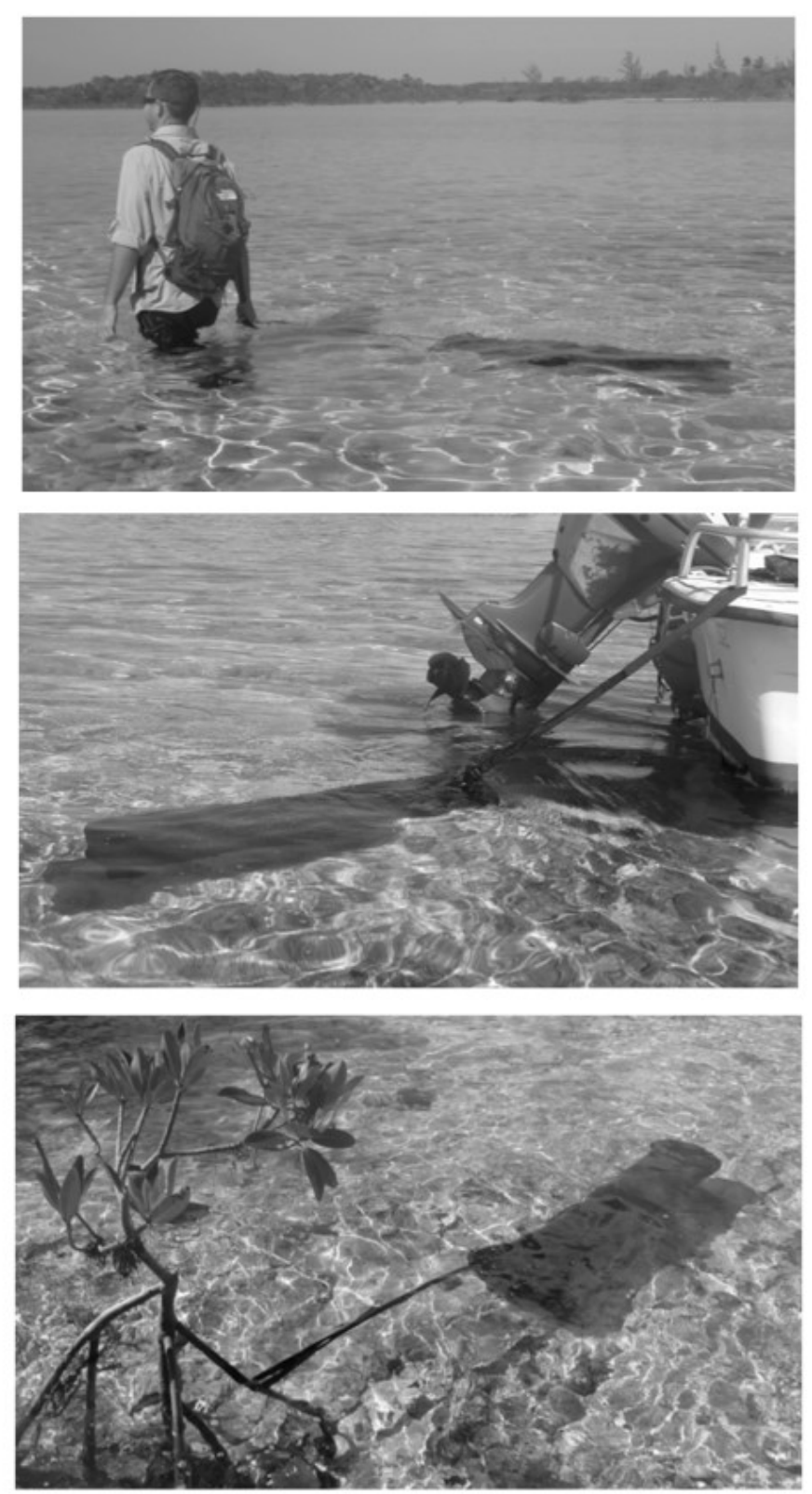
Fig. 5.3: Example of behaviour clusters derived from continuous wavelet transformation and $k$-means cluster analysis, categorized into resting (hatched line), slow swimming (light grey), moderate swimming (grey), and fast swimming (dark grey) behaviours from one bonefish during 30 minutes post-release after simulated angling stress.

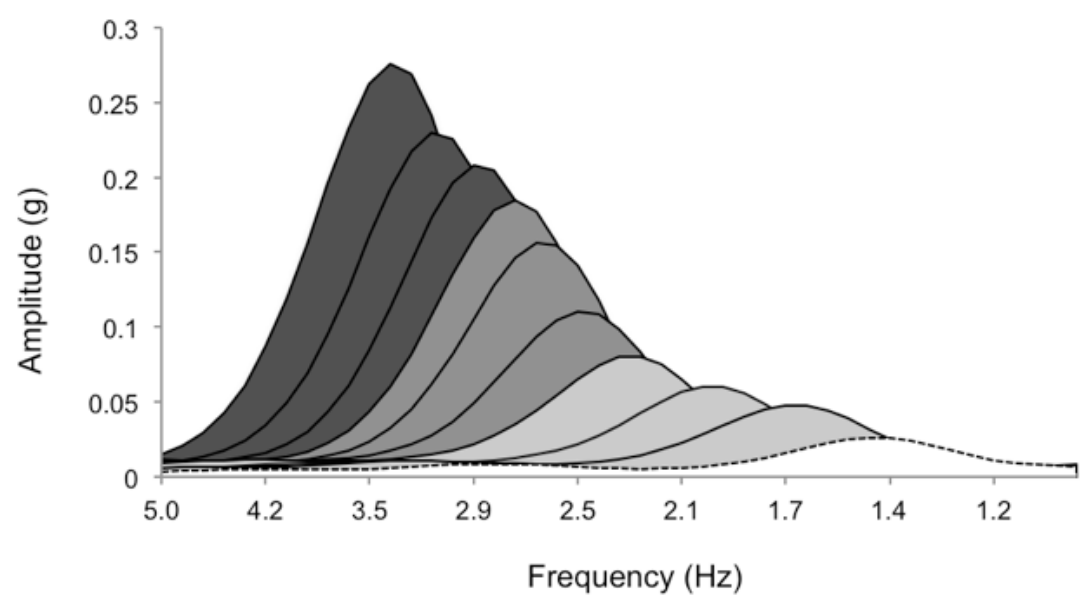




\subsection{Results}

\subsubsection{Validation of reflex impairment indices}

Bonefish exhibited no reflex impairment prior to air exposure, and impairment scores increased with longer air exposure times (Fig. 5.4A). Air exposure times of 2-6 minutes resulted in all bonefish lacking a response to tail grabbing, while nearly all lacked equilibrium, and $80 \%$ of fish lacked body flex response (Fig. 5.4B). No impairment of head complex or VOR was observed after 2 minutes of air exposure. Impairment of head complex was observed after 4 minutes of air exposure, and VOR impairment was observed after 6 minutes.

Based on the responsiveness of bonefish to the RAMP indices, I used them to evaluate the utility of the recovery bags. After air exposure, bonefish released with accelerometers had similar RAMP scores between immediate release $(2.8 \pm 0.14)$ and recovery $(2.7 \pm 0.14)$ treatments. However, after retention in a recovery bag for 15 minutes, all bonefish had RAMP scores of zero (i.e. full recovery).

\subsubsection{Locomotory activity}

There was no significant difference in the length of bonefish in the immediate release $(39.9 \pm 1.1 \mathrm{~cm}$ fork length; mean $\pm \mathrm{SD})$ and recovery $(39.6 \pm 1.0 \mathrm{~cm}$ fork length; mean \pm SD) treatments $(\mathrm{t}=0.20 \mathrm{df}=18 \mathrm{p}=0.86)$. Upon release, recovered bonefish exhibited less locomotory impairment (Fig. 5.5) and left the release site faster than those released 
immediately. Bonefish held in recovery bags had a greater number of tail beats and higher tail beat amplitudes in the first five minutes post-release than those released immediately (Fig. 5.6). When comparing mean tail beat frequencies between treatments in 1-minute intervals for the first 10 minutes, there was a significant interaction between treatment and time $\left(\mathrm{F}_{3,45}=4.13, \mathrm{p}=0.014\right)$. There was also a significant interaction when comparing maximum tail beat frequencies $\left(\mathrm{F}_{4,67}=4.97, \mathrm{p}=0.002\right)$, mean tail beat amplitudes $\left(\mathrm{F}_{2.6,45}=3.92, \mathrm{p}=0.018\right)$, and maximum tail beat amplitudes $\left(\mathrm{F}_{4,68}=6.14, \mathrm{p}\right.$ $<0.001)$. Bonefish retained in recovery bags exhibited significantly higher mean tail beat frequencies in the first 2 min post release, higher maximum tail beat frequencies in the first 3 min, higher mean tail beat amplitudes in the first $3 \mathrm{~min}$, and higher maximum tail beat amplitudes in the first $4 \min (t>1.74, d f=17, p<0.05$ in all cases). Maximum tail beat frequencies and amplitudes remained higher for recovered bonefish than those immediately released for 15 minutes post-release (Fig. 5.6). One bonefish from the immediate release treatment suffered mortality from predation by a $\sim 1.2 \mathrm{~m}$ great barracuda (Sphyraena barracuda) 9 minutes, 40 seconds post-release. No bonefish from the recovery treatment experienced mortality within 30 minutes post-release.

When comparing locomotory activity between treatments during the equivalent time period post-stressor (15-25 minutes post-stressor), there were no significant differences between treatments in tail beat frequencies $\left(\mathrm{F}_{1,17}=0.17, \mathrm{p}=0.69\right)$, maximum tail beat frequencies $\left(\mathrm{F}_{1,17}=0.85, \mathrm{p}=0.37\right)$, mean tail beat amplitudes $\left(\mathrm{F}_{1,17}=0.81, \mathrm{p}\right.$ $=0.38)$, or maximum tail beat amplitudes $\left(\mathrm{F}_{1,17}=0.85 \mathrm{p}=0.37\right)$. However, there was an interaction effect when comparing mean tail beat amplitudes $\left(\mathrm{F}_{3,50}=3.7, \mathrm{p}=0.02\right)$. 
Bonefish held in recovery bags exhibited significantly higher mean tail beat amplitudes 16-17 minutes post-stressor $(\mathrm{t}=2.12, \mathrm{df}=17, \mathrm{p}=0.049)$.

\subsubsection{Post-release behaviour}

Upon release, recovered fish spent relatively more time swimming fast ( $>3$ tail beats/sec, $>0.2 \mathrm{~g}$ amplitudes), while immediately released fish spent more time resting ( $>30 \%$ of first minute) and swimming slowly ( $<2$ tail beats/sec, and/or $<0.1 \mathrm{~g}$ amplitudes) (Fig. 5.7, 5.8). Approximately 4-5 minutes post release, behavioural patterns changed. Bonefish from both treatment groups exhibited similar levels of slow and fast swimming behaviour, while immediately released fish spent more time swimming at moderate speeds (2-3 tail beats/sec, 0.1-0.2 g amplitudes) while located primarily in the main creek channel. Recovered fish began spending more time resting (Fig. 5.8), and the majority of this resting behaviour occurred while bonefish were located along the northern shoreline of Kemps Creek, near rocky outcroppings or red mangrove. 
Fig. 5.4: (A) Reflex action mortality predictor impairment scores (mean \pm SE), and (B) proportion of impairment for five impairment predictors in bonefish after 0 min $(n=30), 2$ $\min (n=20), 4 \min (n=5)$, and $6 \min (n=5)$ of air exposure \pm SE.

A

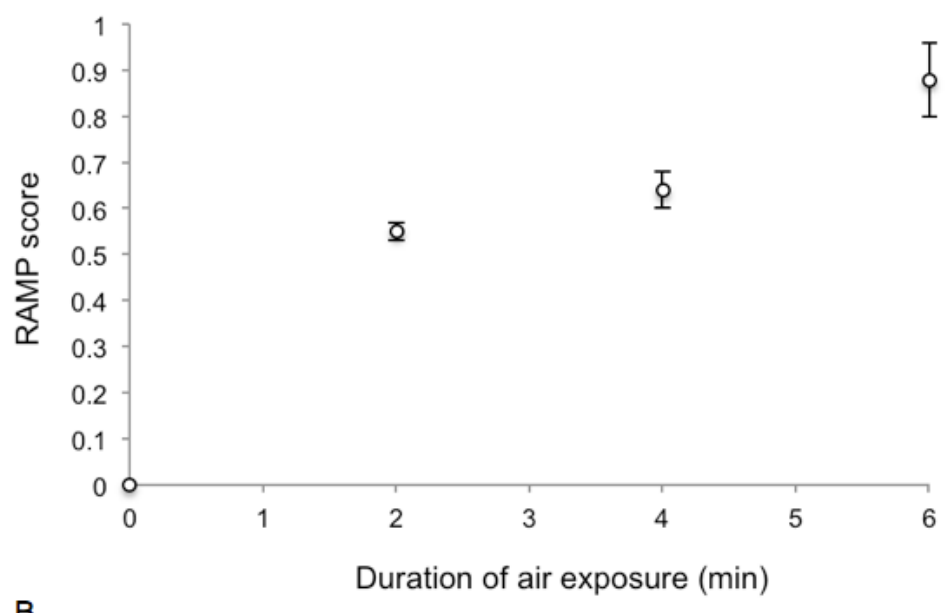

B

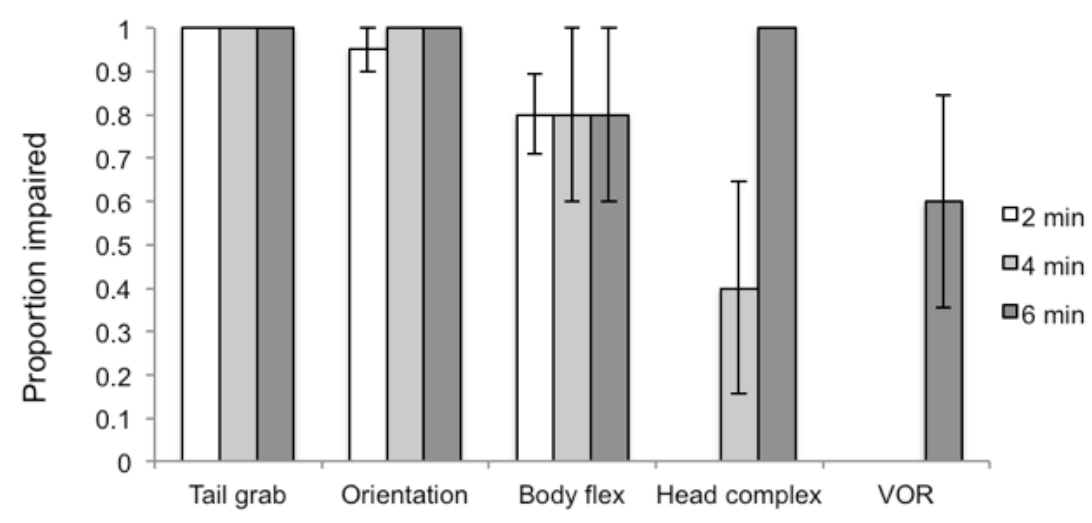

Reflex Action Mortality Predictor 
Fig. 5.5: Bonefish swimming activity (dynamic acceleration, g) in the sway axis measured using tri-axial accelerometers during the first minute post-release after exposure to simulated angling stress and (A) retained in a recovery bag for 15 minutes prior to release, or (B) immediately released. Oscillations represent individual tail beats. Data shown are from the individual bonefish that exhibited median activity levels for their respective treatments in the first minute post-release.
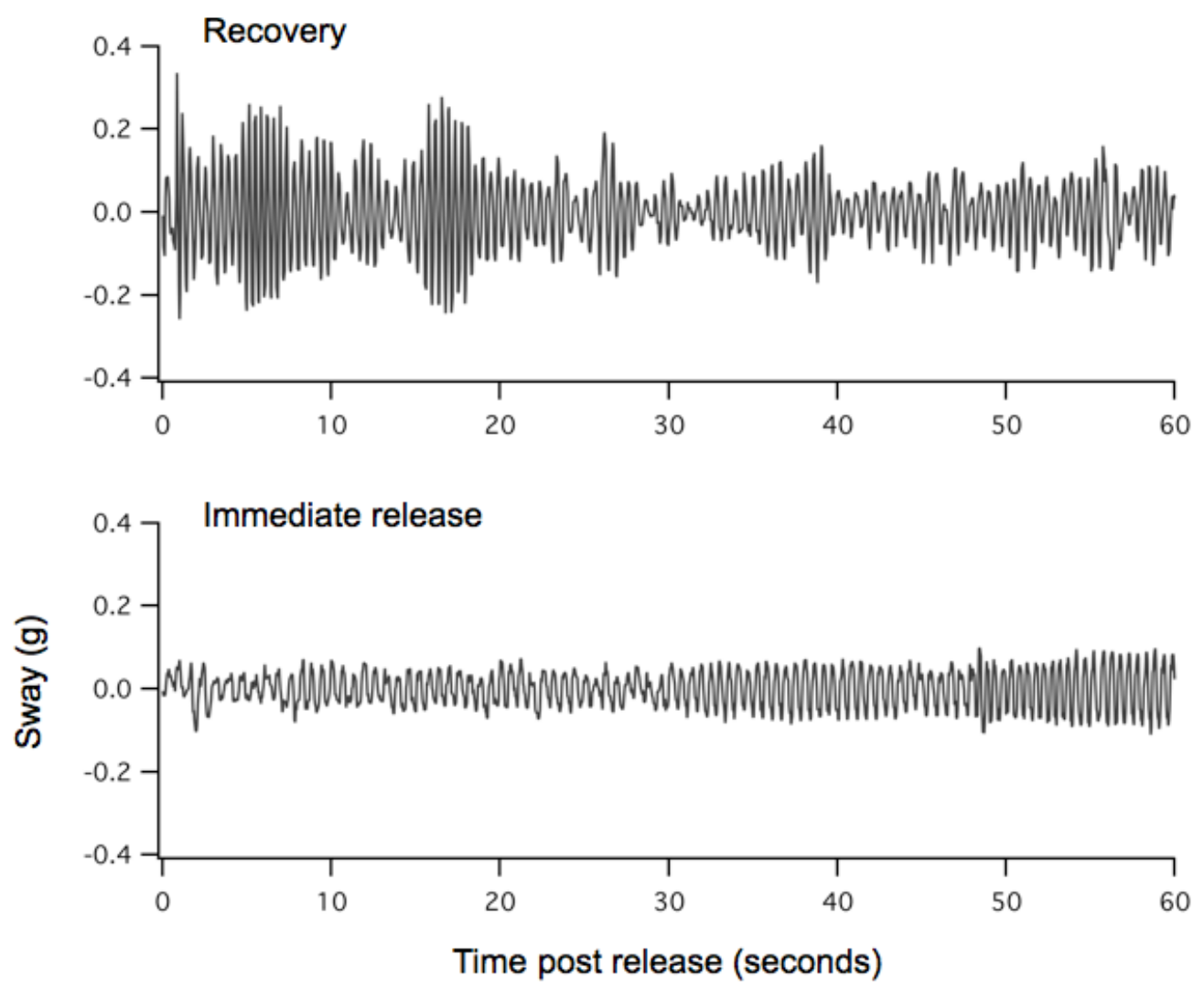
Fig. 5.6: Mean and maximum tail beat frequencies and amplitudes of bonefish for 30 minutes post-release after exposure to simulated angling stress and retained in a recovery bag for 15 minutes prior to release (grey; $\mathrm{n}=10$ ), or immediately released (white; $\mathrm{n}=9$ ) \pm SE. * indicates $\mathrm{p}<0.05, * * \mathrm{p}<0.001$. Tail beat metrics were measured with tri-axial accelerometers and derived from continuous wavelet transformation.
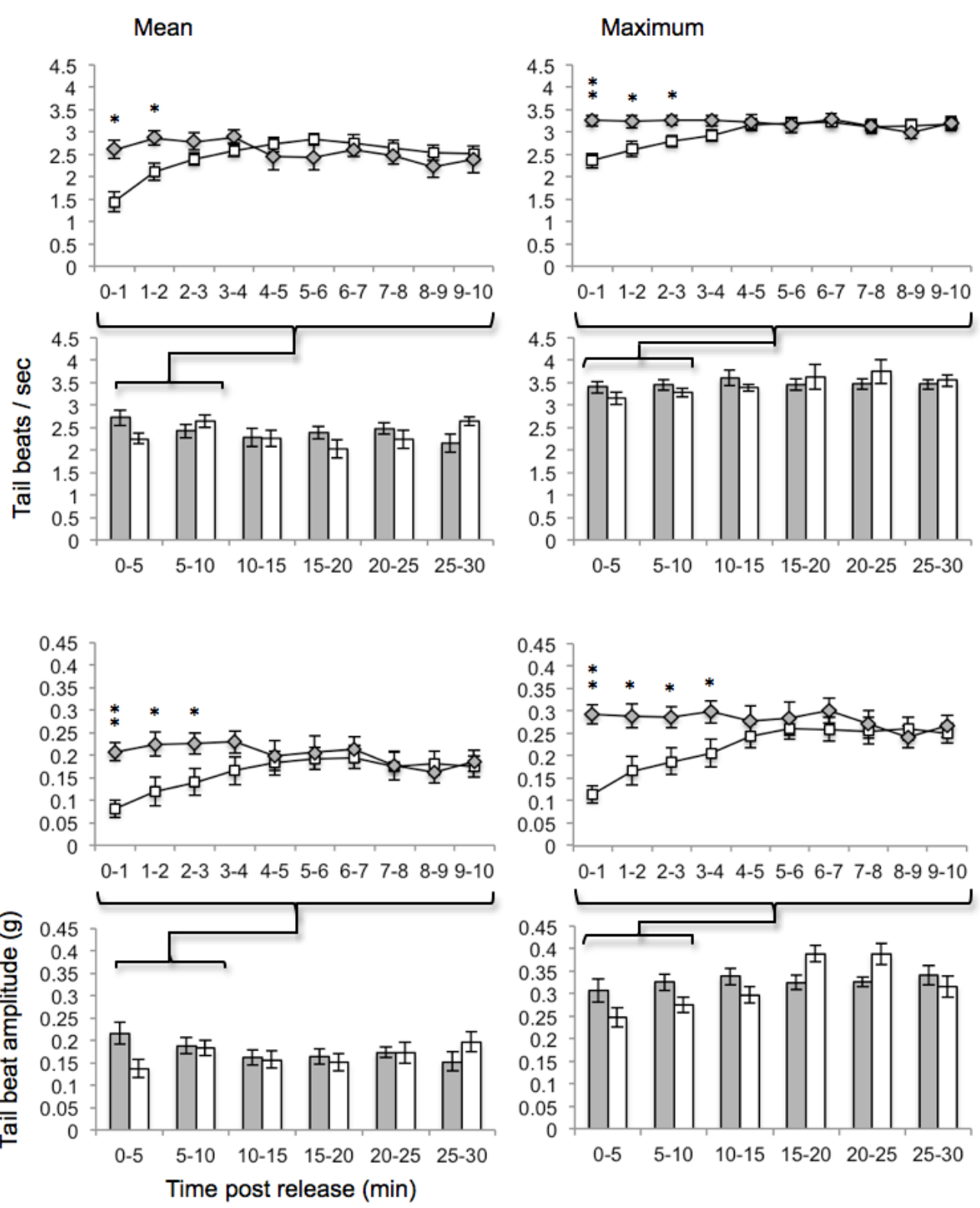
Fig. 5.7: Dynamic acceleration (g) in the sway axis (tail beats) categorized as resting, slow, moderate, and fast swimming behaviours. Data shown is from one bonefish; collected within 30 minutes after angling-related stress.
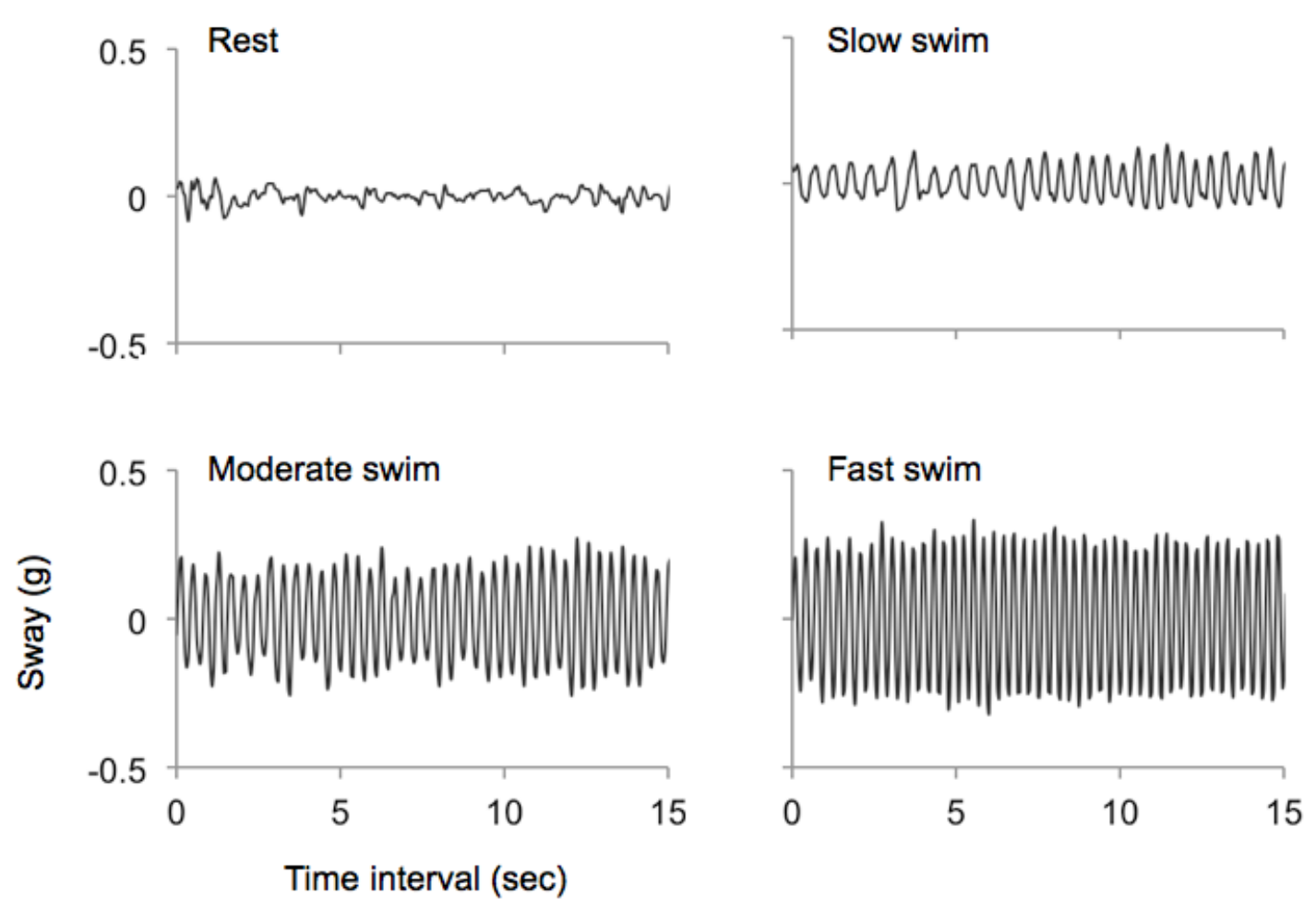
Fig. 5.8: Frequency of bonefish behaviours (mean $\pm \mathrm{SE}$ ) for 10 minutes post-release after exposure to simulated angling stress and retained in a recovery bag for 15 minutes prior to release (grey; $\mathrm{n}=10$ ), or immediately released (white; $\mathrm{n}=9$ ).
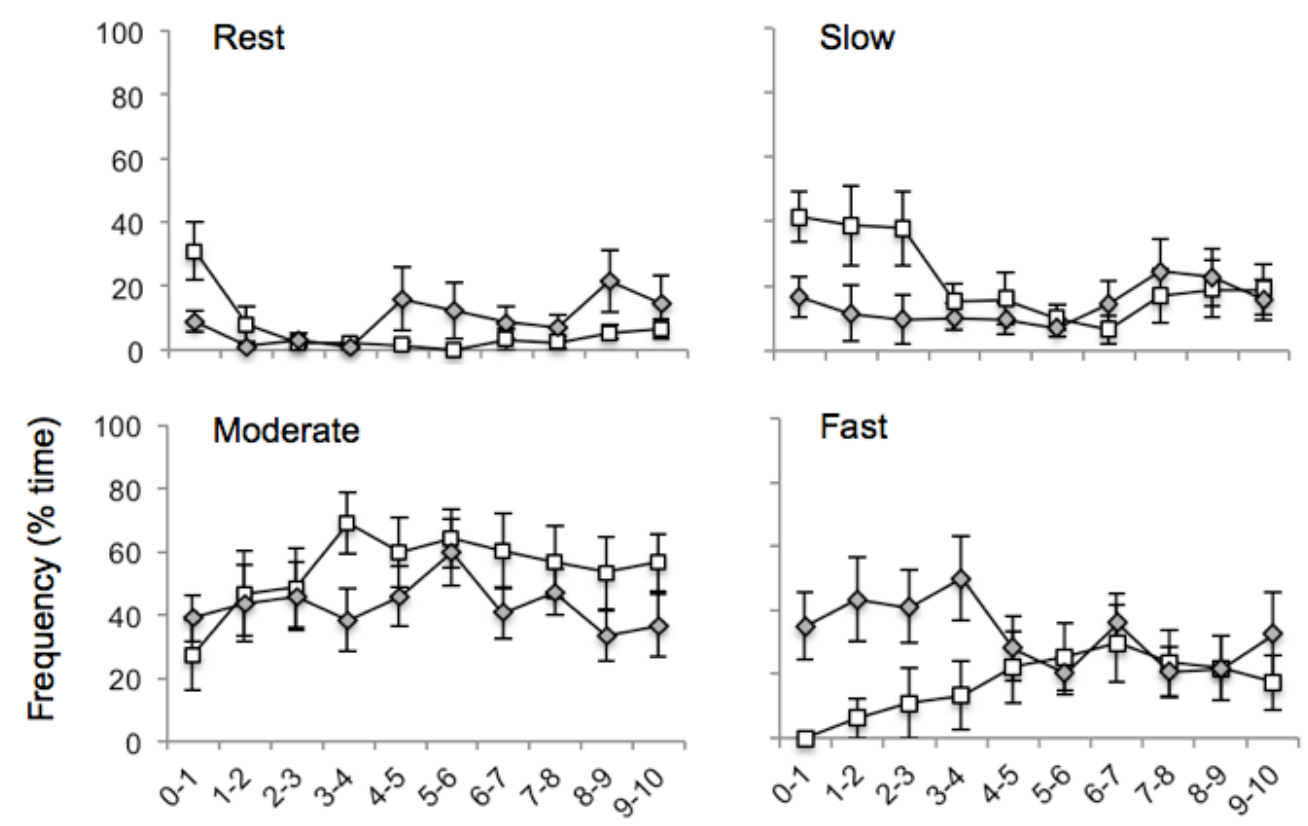

Time post release $(\min )$ 


\subsection{Discussion}

Bonefish that were retained in recovery bags for 15 minutes after angling-related stress exhibited significantly greater locomotory activity in the first 4 minutes post-release than those immediately released, as well as higher maximum tail beat frequencies and amplitudes up to 15 minutes post-release. However, there was little difference in locomotory abilities between treatments when comparing equivalent time periods poststressor, suggesting that recovery bags do not accelerate recovery rates, but provide a means for retaining bonefish; protecting them from predators for the short time period while they recover. Previous studies have shown that nearly all angling-related bonefish predation occurs within 20 minutes post-release, and the majority occurs within the first few minutes (Cooke \& Philipp 2004; Danylchuk et al. 2007a,b). Our results demonstrate that retaining bonefish in recovery bags for 15 minutes reduced locomotory impairment upon release during the critical time period where most predation occurs, and this

practice has the potential to increase survival after catch-and-release angling. Presumably, retaining bonefish in a live well with ambient oxygen levels (Shultz et al. 2011) would have a similar benefit if an angler had access to a boat.

Previous studies have shown that post-release predation of bonefish varies greatly (0-40\%) due to predator abundance (Cooke \& Philipp 2004; Danylchuk et al. 2007a). Predator abundance and thus risk to bonefish post-release appears to vary extensively among sites, seasons, and even years. I observed relatively few potential predators for bonefish during the study aside from a single large barracuda, which is likely the primary 
reason why little post-release predation was observed. Further testing is required to determine whether the reduced behavioural impairments observed here translate into improved survival in more predator rich environments. Similarly, predator abundance is an important consideration for anglers when deciding where and when to release captured bonefish.

Bonefish experience predation after being caught by anglers due to impaired swimming capabilities, and an increased ability of predators to target them through chemical cues (Danylchuk et al. 2007a; Dallas et al. 2010). Given that bonefish excrete the majority of these chemicals shortly after angling events (Dallas et al. 2010), it would be necessary to determine if predators were attracted to recovery bags when holding fish to ensure angler safety. Predators avoided researchers retaining bonefish in recovery bags during this study, however; the only predators present in the study area were juvenile lemon sharks and one large barracuda. Should there be an issue with angler safety in areas with larger sharks, recovery bags could be equipped with magnets, which are effective shark repellants (O'Connell et al. 2010; Robbins et al. 2011).

Bonefish from the respective treatments exhibited very different temporal patterns in post-release behaviour. Bonefish retained in recovery bags initially spent the majority of time swimming at moderate or fast speeds, while those immediately released spent relatively more time resting and swimming slowly. As previously discussed, this difference was likely due to greater locomotory impairment in immediately released fish. Behavioural patterns changed around 4-5 minutes post-release, where immediately 
released bonefish began to spend relatively more time swimming at moderate speeds, and recovered fish spent more time resting. During this time period, bonefish in the recovery treatment spent the majority of their time close to the shoreline, near rocky outcroppings or mangroves. It seems that recovered bonefish were using these areas as a refuge for rest and recovery. Conversely, immediately released fish spent the majority of their time swimming at moderate speeds in the main creek channel, or on the flat adjacent to the creek. Indeed, the one bonefish that was depredated during this study was located in the main creek channel. A previous study also found that immediately released bonefish did not use available structures refuge (Danylchuk et al. 2007a). Animals are known to exhibit inhibited decision-making abilities after acute stress (Shafiei et al. 2012; Starcke \& Brand 2012). Our results suggest that retaining bonefish for a short period prior to release may actually facilitate improved decision-making in terms of improving recovery and reducing predation risk. Previous studies have shown that angled northern pike (Esox lucius) also rest in refuge habitats after release, and experience little short-term mortality (Klefoth et al. 2008; Arlinghaus et al. 2009). However, in the case of bonefish, the ability to utilize shoreline structures for refuge likely depends on the size and location of predators, and the complexity of shoreline habitats (Danylchuk et al. 2007a).

Accelerometer loggers are novel instruments for measuring fine-scale behaviour and energy expenditure of animals in the wild (Wilson et al. 2008; Sakamoto et al. 2009; Whitney et al. 2010; Wilson et al. 2012). To our knowledge, this study is the first to use tri-axial accelerometry to measure the effects of a disturbance event on animal behaviour, and in relation to angling interactions (Donaldson et al. 2008). Accelerometer loggers 
provided detailed and quantifiable information on bonefish activity after angling-related stress, which allowed for greater insight into how retaining bonefish in recovery bags reduces behavioural impairments. To accomplish this, I applied recently developed data analysis techniques to estimate tail beat frequencies and amplitudes, and grouped this information into behaviour types. While overall dynamic body acceleration (ODBA) is currently the best proxy for energy expenditure (Gleiss et al. 2011), I elected to use tail beat metrics as an indication of bonefish vitality because post-release predation is due to behavioural impairments (Cooke \& Philipp 2004; Danylchuk et al. 2007a). The ability of bonefish to evade predators likely depends not only on their ability to move and expend energy, but to swim in a coordinated manner.

The five impairment indicators I tested on bonefish provided a gradient in impairment scores that related to the degree of stressor (i.e., 0-6 min of air exposure). RAMP scores have been correlated with stressor duration and mortality for a number of fish species (Davis 2005, 2007; Davis \& Ottmar 2006; Humborstad et al. 2009; Raby et al. 2012). Indeed, the duration of a stressful event increases the level of physiological disturbance in bonefish (Suski et al. 2007; Cooke et al. 2008), while longer handling times and air exposure durations result in higher post-release predation rates (Danylchuk et al. 2007a). In this study, bonefish that were equipped with accelerometers exhibited moderate impairment scores after 2 minutes of air exposure, while no impairment was detected after 15 minutes of retention in a recovery bag, and fish from the recovery treatment exhibited significantly higher levels of activity upon release. Therefore RAMP scores appear to be a good indication of bonefish vitality. Bonefish anglers may be able 
to use RAMP to assess bonefish condition, and make educated decisions on whether to release the fish, or retain it for a short period to facilitate recovery. Likewise, if water temperatures and bonefish impairment scores are very high, responsible anglers can recess until conditions are more favourable.

The impairment indicators tail grab, equilibrium, and body flex were the first to become impaired in bonefish, and impairment levels within these predictors did not vary with increased stress duration. This was likely because bonefish were all highly impaired at the lowest level of stress I inflicted. Indeed, a previous study found roughly $50 \%$ of bonefish lose equilibrium after angling events (Danylchuk et al. 2007a), while 95\% of bonefish lost equilibrium after simulated angling stress ( 2 minutes of air exposure) in this study. These three predictors may provide an indication of impairment levels with lesser degrees of stress. Head complex was the next to become impaired at 4 minutes of air exposure, followed by VOR at 6 minutes. Therefore head complex and VOR predictors are indicative of very high levels of physiological disturbance in bonefish. This predictorspecific pattern of impairment in bonefish is nearly identical to that of coho salmon (see Raby et al. 2012).

In conclusion, retaining bonefish in recovery bags for 15 minutes prior to release after simulated angling stress reduced locomotory impairment during the critical time period where post-release predation normally occurs. This practice may also promote resting behaviour in possible refuge areas, further facilitating recovery and avoidance of predation. Recovery bags may also have potential to reduce mortality for other species 
that experience mortality after angling due to predation, such as the red snapper (Lutjanus campechanus) (Parker 1985). They also have potential for improving survival after other stress events, and have been shown to reduce reflex impairment and improve survival of migrating salmonids after being caught in commercial fishing nets (Donaldson et al. 2013). The impairment index tested here provided a gradient of impairment scores that were correlated to the level of stressor. Further testing should use real angling events rather than angling-related stressors (as I used here) to evaluate the extent to which these findings apply to C\&R angling (Cooke et al. 2013a). Moreover, work to identify the optimal duration of recovery would be useful to ensure maximal benefit to the fish while minimizing effort by the angler. Finally, the use of tri-axial accelerometer loggers served as a novel tool for documenting behavioural impairments associated with angling interactions and is worthy of consideration for future C\&R studies. 


\section{Chapter 6. Fishing-related stressors inhibit refuge-seeking behaviour in released sub-adult great barracuda}

\subsection{Abstract}

Fisheries interactions that result in the capture and release of fish can cause behavioural impairments that increase the risk of post-release predation. Increased predation risk has been attributed to reduced swimming capabilities, however the role of refuge (structural shelter) use is still poorly understood. Here, I examined the effect of fishing-related stressors on the ability of sub-adult great barracuda Sphyraena barracuda to attain refuge in an experimental wetland in Eleuthera, The Bahamas. Great barracuda were either treated as controls with minimum stress $(n=7)$, chased to exhaustion $(n=8)$, or chased to exhaustion and air exposed for $90 \mathrm{sec}(\mathrm{n}=7)$. Reflex Action Mortality Predictors (RAMP) including ability to maintain equilibrium and bursting response were assessed prior to release at a standardized location. Red mangrove Rhizophora mangle prop roots provided refuge $\sim 5 \mathrm{~m}$ from the release point. Great barracuda were visually monitored for up to 30 min post-release to quantify behaviour (resting, swimming, directional changes) and the time when refuge was reached. RAMP scores increased with the degree of stressor, and fish in both fishing-related stressor treatments took significantly longer to reach refuge than control fish, while air exposed fish took significantly longer than fish that were only chased. Prior to reaching refuge, fish exposed to fishing-related stressors spent less time swimming, and changed directions more frequently than control fish, indicating that impaired locomotory capabilities and disorientation inhibited refuge use. The inability to 
find refuge after an acute stressor such as capture and release fisheries may be an important factor contributing to post-release mortality for relevant species.

\subsection{Introduction}

No matter the fisheries sector, a component of the catch is released. In commercial fisheries, non-target individuals are often regarded as bycatch and thus discarded (Hall et al. 2000; Hall \& Mainprize 2005), a phenomenon that is exceedingly common (Davies et al. 2009). In the recreational sector, catch-and-release angling is increasing in popularity due to a combination of harvest regulations and the conservation ethic of anglers (Cowx 2002; Cooke \& Cowx, 2004; Arlinghaus et al. 2007). Although the release of fish from various fisheries is certainly a conservation strategy (Cooke \& Cowx 2006), released fish may have reduced fitness due to physical injuries, energetic costs of recovery from physiological disturbance, or behavioural impairments (Davis 2002; Arlinghaus et al. 2007; Cooke \& Schramm 2007). In certain environments, these behavioural impairments cause some species to be more vulnerable to predators (reviewed in Raby et al. 2014).

Fisheries interactions are often associated with elevations in oxygen consumption, depletion of tissue energy stores, and associated physiological alterations (reviewed in Davis 2002; Cooke \& Suski 2005), that can cause behavioural impairment post-release (Cooke et al. 2014b). Negative behavioural impacts are often attributed to reduced locomotory capabilities (Cooke et al. 2000) although recent studies on Bonefish Albula vulpes, suggest that refuge (structural shelter) use may also be affected, contributing to 
predation risk (Danylchuk et al. 2007; Chapter 5). Cooke et al. (2014b) found that fisheries-related stressors caused cognitive impairment in Spanish Flag Snapper Lutjanus carponotatus, which precluded their ability to locate and/or evaluate potential refuge habitats up to $30 \mathrm{~min}$ post stressor. In general, behaviour has rarely been used as an endpoint in capture and release fisheries, which is likely driven by the fact that most studies focus solely on injury or mortality as endpoints. Clearly there is opportunity to extend studies of fisheries interactions to consider potential behavioural impairments in both laboratory and field environments given the need to improve our understanding of ecologically relevant endpoints such as behavior (Cooke et al. 2014a).

Great barracuda Sphyraena barracuda are a common predatory fish in tropical and sub-tropic oceans worldwide (de Sylva 1963). It is a popular species for recreational anglers, caught as a target species and incidentally. In addition, commercial and artisanal fisheries frequently capture barracuda using a variety of gear types, including hook and line (e.g., rod and reel, hand-lining). Barracuda have the potential to biomagnify ciguatera bacteria, further elevating release rates (Villareal et al. 2007; O'Toole et al. 2010). Great barracuda live in environments with high predator burden, and smaller fish in particular may be more vulnerable to predation post-release by larger great barracuda or sharks. Sub-adult great barracuda often occupy shallow nearshore areas such as reefs and mangrove creeks, using these structurally complex habitats for refuge (de Sylva 1963; Paterson 1998). Therefore sub-adult great barracuda offered a good model for testing if and how fishing-related stressors influence refuge-seeking behaviour. 
The objective of this research was to examine the influence of fishing-related stressors on behaviour and refuge use in sub-adult great barracuda. To do so, I used two common fishing-related stressors, exhaustive physical exercise and air exposure, and quantified behavioural impairment with a well-established index, Reflex Action Mortality Predictors (RAMP; Davis 2010; Raby et al. 2012) prior to release in an enclosed experimental wetland environment. I predicted that great barracuda exposed to fishingrelated stressors would take longer to reach refuge due to reduced locomotory abilities, as well as non-directional swimming associated with disorientation.

\subsection{Methods}

\subsubsection{Fish collection}

Sub-adult great barracuda were collected by seine net from Page Creek $\left(24.8212^{\circ} \mathrm{N}\right.$, $76.3106^{\circ} \mathrm{W}$ ) in Eleuthera, The Bahamas on 19-01-2013 and brought to the Cape Eleuthera Institute for experimental testing. Great barracuda were held in circular tanks (3.7 m diameter, $1.25 \mathrm{~m}$ height; $13180 \mathrm{~L}$ ) for $24-48$ hours prior to experimentation.

\subsubsection{Stress and refuge experiments}

Great barracuda were subjected to one of three treatments prior to release into an enclosed $\sim 2500 \mathrm{~m}^{2}$ wetland (see Murchie et al. 2011a) via an 8m waterslide. Control fish $(\mathrm{n}=7 ; 29 \pm 3.0 \mathrm{~cm} \mathrm{FL})$ were released with minimal stress (aside from handling) prior to 
release. Chased to exhaustion treatment $(\mathrm{n}=8 ; 28.4 \pm 3.4 \mathrm{~cm} \mathrm{FL})$ were chased by hand in a tote $(0.8 \times 0.5 \times 0.5 \mathrm{~m})$ until they no longer responded to tail grabbing. The chase and air exposure treatment $(\mathrm{n}=7 ; 27.1 \pm 5.4 \mathrm{~cm} \mathrm{FL})$ were chased by hand to exhaustion and exposed to air for $90 \mathrm{sec}$ in a rubberized mesh net. Prior to release, great barracuda were assessed for five RAMP indicators, including tail grab, equilibrium, body flex, head complex, and VOR (see Chapter 5). Tail grab response was assessed by grabbing the fish's tail while in water; it was considered impaired if the fish did not attempt to swim away. Fish were considered to have lost equilibrium if, when rolled upside down, they failed to right themselves within $3 \mathrm{sec}$. Body flex was assessed by holding the fish in air by the middle of body; a lack of attempt to struggle indicated impairment. While held in air, a lack of regular opercular beats indicated impairment of head complex. Lastly, VOR was assessed by rolling fish side to side, and was considered impaired if their eyes did not maintain the same pitch and track the handler. Each indicator was scored as either 1 (impaired) or 0 (unimpaired), with higher RAMP scores indicating greater impairment. Upon entry to the wetland, great barracuda were visually monitored from a raised walk bridge that stretches across the wetland for up to $30 \mathrm{~min}$ to record behaviour (resting, swimming, directional changes), and time to refuge. Directional changes were defined as a $90^{\circ}$ alteration in bearing by the fish. Distance to potential refuge was $\sim 5 \mathrm{~m}$ in all directions, which was red mangrove Rhizophora mangle, in three directions, and the walking bridge in the fourth. 


\subsubsection{Data analysis}

One-way ANOVA was used to compare the sizes (FL) of great barracuda, and there were no significant differences between treatments $\left(\mathrm{F}_{2,19}=0.33, \mathrm{p}=0.72\right)$. Cox proportional hazard regression analysis was used to determine whether the amount of time until Great Barracuda attained refuge varied significantly between treatments. The percentage of time spent swimming prior to reaching cover, as well as the number of direction changes made by great barracuda were compared across treatments using Kruskal-Wallis ANOVAs with Bonferonni corrected pairwise comparisons. Assumptions of normality, and for Cox regression, proportionality and nonlinearity were checked prior to analysis, and the level of significance was $\mathrm{p} \leq 0.05$. All analysis was conducted using RStudio ( $R$ Core Team, 2012).

\subsection{Results}

Great barracuda RAMP scores increased consistently with the degree of stressor, with a mean score of $0 \pm 0$ for control fish, $2.4 \pm 0.5$ for fish chased to exhaustion, and $3.4 \pm 0.5$ for fish chased to exhaustion and air exposed (Fig. 6.1). All great barracuda but one individual eventually utilized red mangrove as refuge within $30 \mathrm{~min}$ post-release. The time until great barracuda reached refuge varied significantly between treatments $\left(\chi^{2}(2)=19,7, p<0.001\right.$; Fig. 6.2). Great barracuda in the chase treatment took significantly longer to reach refuge than the control treatment $(z=2.7, p=0.006$, hazards ratio $=7.5)$, as did great barracuda that were chased and air exposed $(\mathrm{z}=3.8, \mathrm{p}<0.001$, hazards 
ratio $=44.3$ ). Chased and air exposed fish also took longer to reach refuge than fish that were only chased $(\mathrm{z}=2.2, \mathrm{p}=0.03$, hazards ratio $=5.9)$.

Prior to reaching cover, there was a significant difference between treatments in time spent swimming $\left(\mathrm{H}_{2}=6.0, \mathrm{p}=0.04\right)$. Control fish spent significantly more time swimming than fish chased and air exposed $(\mathrm{p}<0.05$; Fig. 6.3a) but not fish that were only chased. There was also a significant difference in the number of direction changes made prior to reaching cover across treatments $\left(\mathrm{H}_{2}=7.2, \mathrm{p}=0.03\right)$. Both fish chased to exhaustion and those chased and air exposed made significantly more direction changes per minute than control fish ( $<<0.05$, Fig. $6.3 b)$. 
Fig. 6.1: Great barracuda RAMP scores across three treatments prior to release into the wetland.

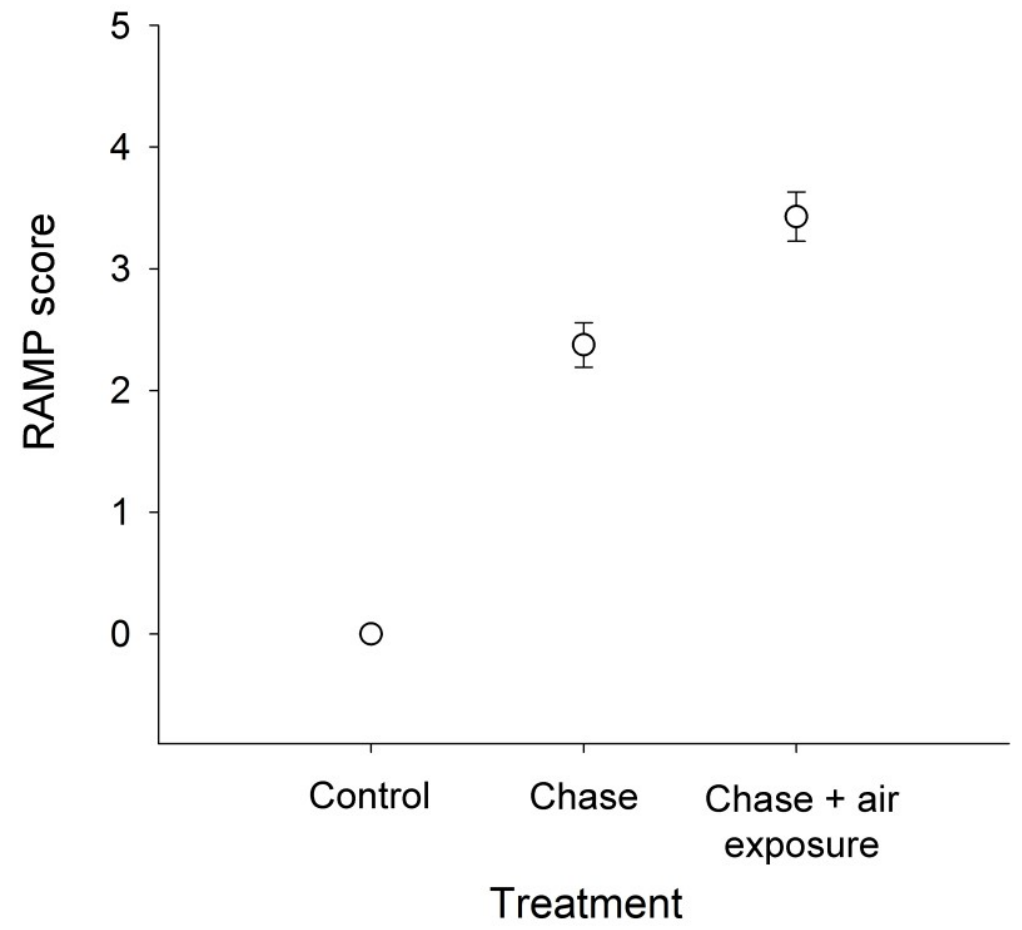


Fig. 6.2: Proportion of great barracuda in refuge with time post-release into the wetland across treatments $($ Control $=$ solid, Chase $=$ dashed, Chase + air exposure $=$ dotted $)$.

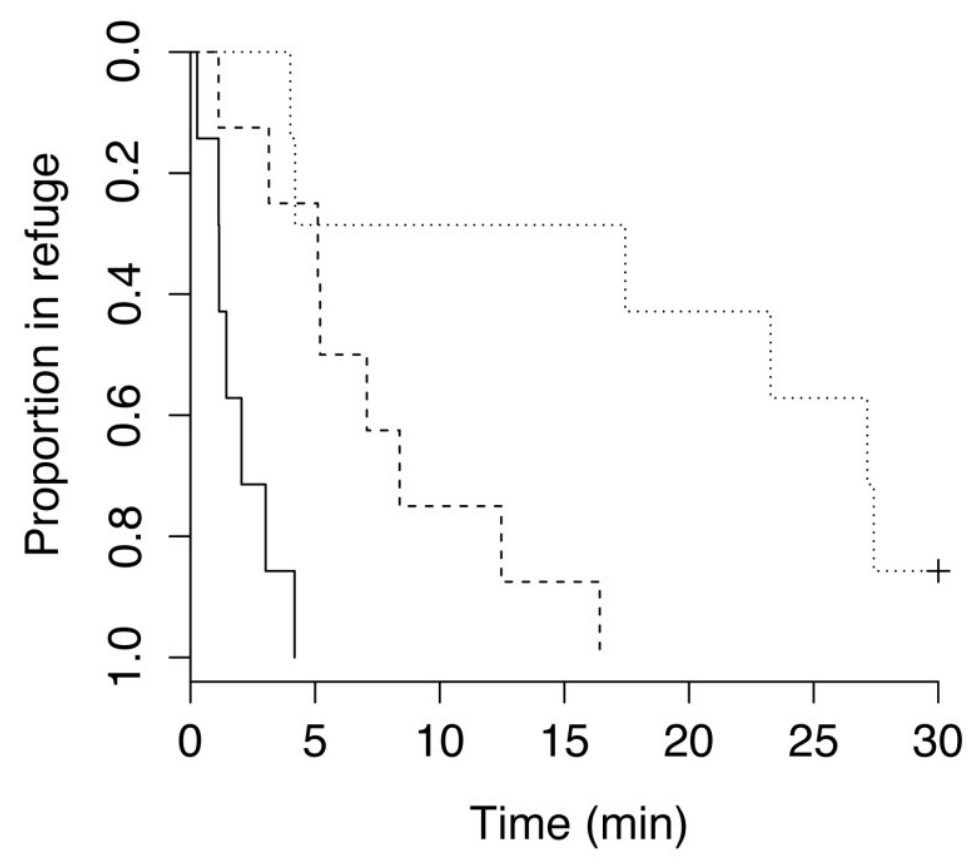


Fig. 6.3: (A) Time spent swimming (\%) by great barracuda, (B) Number of direction changes/min by great barracuda across three treatments prior to reaching cover in the wetland. Dissimilar letters indicate significant differences.

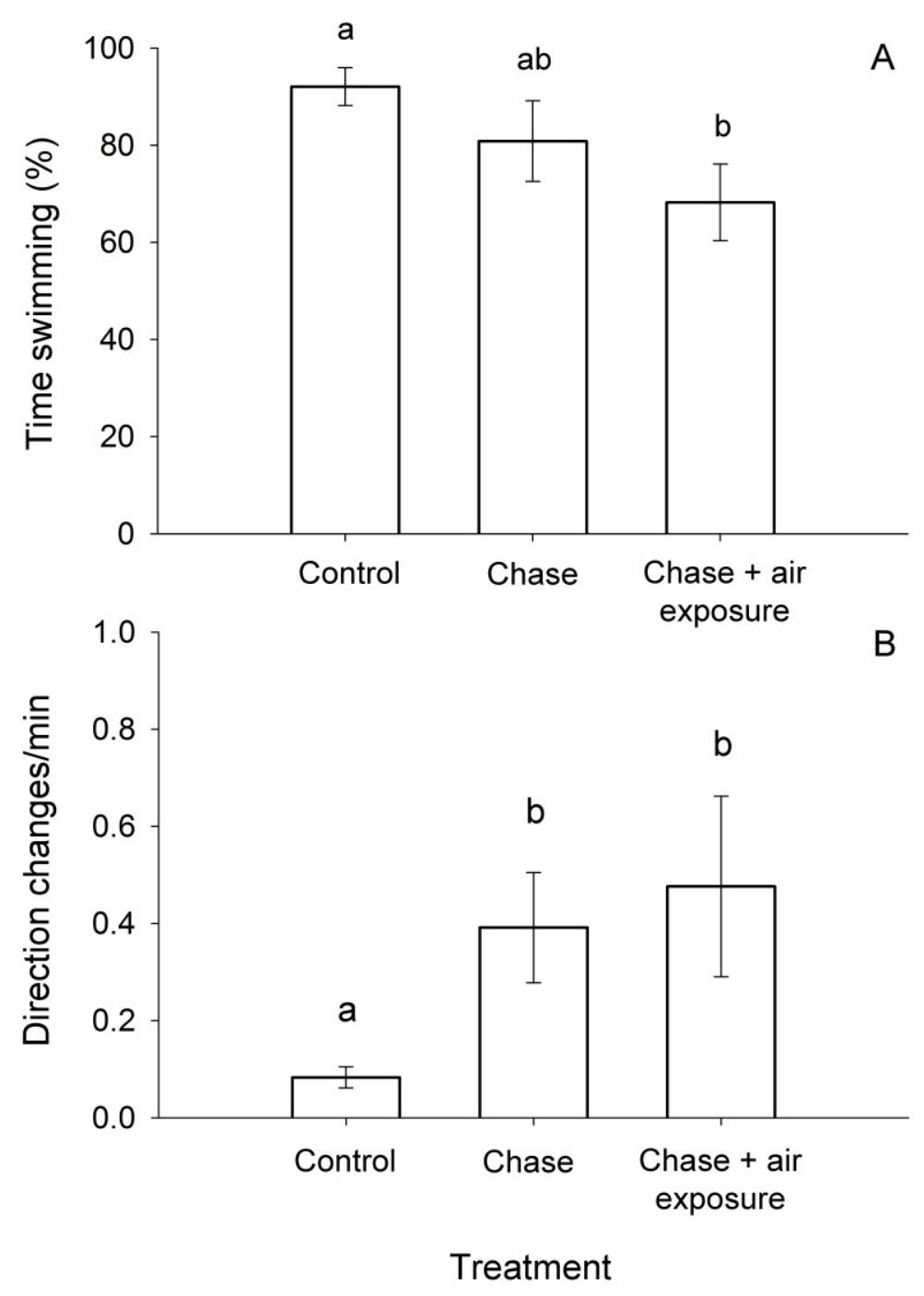




\subsection{Discussion}

Great barracuda exposed to fishing-related stressors had higher reflex impairment (RAMP) scores and took significantly longer to reach refuge in the wetland than control fish. Juvenile and sub-adult great barracuda are known for using mangrove and reef structure for refuge (de Sylva 1963; Paterson 1998). Indeed, the great barracuda in this study were collected from red mangrove habitat, and fishing-related behavioural impairment had a significant impact on their ability to return to similar refuge upon release in the experimental wetland. It is well established that fisheries-related stressors cause behavioural impairment that is often correlated with mortality (Davis 2010; Raby et al. 2012), which in some ecosystems commonly involves opportunistic predators (Raby et al. 2014). While there were no predators of great barracuda in the wetland during this study, the longer they spend in open areas away from refuge, the greater the potential risk of predation (e.g., birds, conspecifics, sharks) in the wild. This research supports previous assertions that behavioural impairments can impact refuge use, which may contribute to higher predation risk, particularly in environments with high predator burden (e.g. shallow tropical or sub-tropical seas; Danylchuk et al. 2007a; Raby et al. 2014).

While in search of refuge, great barracuda exposed to fishing-related stressors spent less time swimming and made more directional changes than control fish, suggesting that delayed refuge use results from both locomotory impairment and disorientation. Indeed, Cooke et al. (2014b) also found fishing-related stressors affected refuge-seeking behaviour of a predatory reef fish, the Spanish flag snapper, which took 
longer to find refuge and were more apprehensive to utilize it. Stress causes physiological disturbances that impact the function of cognitive, sensory, and locomotory systems (Wendelaar Bonga 1997; de Kloet et al. 1999; Starcke \& Brand 2012). Fishing-related stressors can reduce the ability of fish to locate refuge areas in addition to being less physically capable of swimming there and avoiding any potential predatory attacks.

Great barracuda that were chased to exhaustion without being air exposed were intended to represent a relatively gentle fishing-related stressor. This treatment exhibited moderate reflex impairment, with $25 \%$ losing equilibrium, which has been shown to be a significant predictor of predation risk in bonefish (Danylchuk et al. 2007a). Furthermore, while fish in this treatment did not spend significantly less time swimming than control fish, they made significantly more directional changes. Impaired cognitive or visual acuity may be an important contributor to predation risk in even moderately stressed individuals. Great barracuda that were also air exposed, representing a harsher fishing stressor, had higher reflex impairment and took significantly longer to reach cover than both Control and Chased treatments due to both impaired locomotory capabilities and disorientation. Cooke et al. (2014b) revealed that even highly stressed Spanish flag snapper regain the ability to attain refuge 30 min post-release regardless of maintaining high levels of physiological stress. This corroborates with our findings, where even highly stressed barracuda all found cover within a 30 min period with the exception of one individual. When fish exhibit high levels of reflex impairment after fishing-relating stress, retention in livewells, recovery bags, or even simple plastic containers with ambient oxygen levels (Shultz et al. 2011; Donaldson et al. 2013; Cooke et al. 2014b) for 
a short time period may improve survival by providing a short time period for recovery of locomotory, cognitive, and visual faculties.

In summary, fishing-related stressors caused behavioural impairment that influenced the ability of great barracuda to reach refuge habitat, likely due to a combination of impaired locomotion and disorientation. This effect is consistent with the degree of fishing-related stress, which can be effectively measured with reflex impairment indices (RAMP). Disorientation may be a particularly important factor contributing to inhibited refuge use and higher predation risk in fish with moderate impairment. 


\section{Chapter 7. Synthesis and future research directions}

The overarching hypothesis for my dissertation was that ecological and anthropogenic factors influence animal behaviour and energetics. Different aspects of this hypothesis are supported in each of the five data chapters. Chapters 2-4 find supporting evidence for the influence of ecological factors on bonefish behaviour and energy expenditure in a seminatural wetland, as well as in the wild from fine to broad spatial scales, with some interesting consistencies (or lack thereof) across these scales. Chapters 5 and 6 find support for the influence of an anthropogenic activity, recreational angling, on the postrelease behaviour of bonefish and great barracuda in the context of post-release predation risk, providing insight into the mechanisms behind stress related predation risk, and some exploration of potential remediation tactics. These findings have important implications for behavioural and physiological ecology, and bioenergetics modeling, as well as resource management and conservation. Here I integrate these findings into a broader framework in the form of a synthesis, focusing the discussion around key themes.

\subsection{Ecological influences on behaviour and energetics}

In this dissertation I determined that landscape features had the greatest influence on bonefish energy expenditure at both fine (Chapter 3) and broad (Chapter 4) spatial scales. Further, energy expenditure was driven mainly by activity levels, more so than temperature, indicating that bonefish were behaving differently across the habitat mosaic at both spatial scales. This finding is not surprising; different habitats often serve 
different functions for organisms, whether for foraging, transitional movement, refuge from predators, or reproduction (Morrison et al. 2012). The spatial patterns in energy expenditure observed here likely reflect bonefish behavioural ecology; bonefish move in and out of shallow, nearshore areas to forage with the flooding and ebbing tides, respectively (Murchie et al. 2013). Interestingly, bonefish energy expenditure tended to correlate negatively with water depth at very fine spatial scales in Culebra, but correlate positively with water depth a broader scales in both Culebra and Eleuthera. This is certainly supporting evidence for the importance of scale in studying ecology (Levin 1992). Presumably the high activity levels and energy expenditure in deeper water reflect fast movements through nearshore coastal areas between shallow water habitats, which likely offer both increased foraging opportunities and reduced predation risk (McIvor \& Odum 1988; Clark et al. 2003; Rypel et al. 2007). Movement from shallow water habitats through nearshore areas may be related to migration to deeper water habitat, where bonefish spawn (Danylchuk et al. 2011). Conversely, at finer spatial scales, bonefish also expend high levels of energy in very shallow habitats in Culebra (i.e., reef crest), which may also serve as a movement corridor between the fore reef habitat on the ocean side, and seagrass on the lagoon side of the reef. From an applied perspective, habitat function is an important consideration for managing habitat (e.g., marine protected areas) and anthropogenic activities (e.g., fishing). For example, regulations could limit fishing practices during spawning migrations, when fish are often aggregated and more vulnerable to capture (Coleman et al. 1996; Demitcheson et al. 2008). 
Diel period is generally the greatest influence on circadian rhythms in animals, which drives patterns of wakefulness, activity, and foraging (Boujard et al. 1992). Different species or populations of animals are active at specific times of day, categorized as diurnal, nocturnal, or crepuscular. In some species activity and foraging behaviour can be highly flexible, depending on temporal variability in factors such as temperature and prey availability (Fraser et al. 1993; Metcalfe et al. 1999). In the enclosed wetland (Chapter 2), bonefish tended to be most active at dawn, followed by day, night, and lastly dusk. In the wild, bonefish were also more active, expending more energy during the day than at night at both fine and broad spatial scales (Chapters 3,4 ). While I would categorize bonefish generally as a diurnal species, there is certainly diversity among individuals, with some being more active at night. Moreover, In Chapter 2, the majority of individuals foraged most often at night. Future research could explore the intrinsic (e.g., fish size, metabolic rate, sex) and extrinsic (e.g., competition, habitat use) factors that influence this inter-individual variation and intra-individual consistency in locomotory activity. While my sample sizes throughout this dissertation were not large enough to explore intra-individual correlations in traits such as activity and foraging rates, the diversity of behavioural tendencies observed here lays a foundation for further research on the topic.

Environmental temperature is recognized as one of the most important environmental factors influencing animal behaviour and energy expenditure (Clark et al. 1999; Seibel \& Drazen 2007). This is particularly true for ectotherms because temperature controls the rate of metabolic processes and fitness-related performance (Fry 
1947; Brett 1979; Pörtner \& Lannig 2009). In Chapter 2 I found water temperature was a significant predictor of bonefish activity levels, which generally peaked around $25-27^{\circ} \mathrm{C}$. This is corroborated in Chapter 3, where bonefish activity levels on a fringing reef in Culebra, Puerto Rico were highest between $25-30^{\circ} \mathrm{C}$, and declined at temperatures exceeding $30^{\circ} \mathrm{C}$. These findings are consistent with the aerobic scope for activity in bonefish established in laboratory experiments, which peaked $\sim 27^{\circ} \mathrm{C}$ and rapidly declined above $30^{\circ} \mathrm{C}$ (Nowell et al. 2015). This is also coherent with bonefish spatial movements, as bonefish selectively used the shallow nearshore fringing reef (Chapter 3) based on seasonal variability in water temperatures. More specifically, bonefish moderated energy expenditure across seasons through selective use of nearshore habitats when temperatures were moderate and their aerobic scope was near optimal. Based on the oxygen- and capacity-limited thermal tolerance concept, as ectotherms, occupying temperatures that are optimal for aerobic scope enables fish to accomplish fitness related activities including movement for foraging or predator avoidance, as well as somatic growth and gamete development (Pörtner \& Lannig 2009). Temperature very likely influences the timing of nearshore foraging movements by bonefish for this reason. In shallow tropical waters, temperature can be highly variable between night and day. Given that bonefish avoid shallow flats when water temperatures are very high, there could be an interaction between season and diel period in predicting occupancy of nearshore habitats by bonefish. I would predict that bonefish utilize nearshore flats more at night during the summer and autumn seasons when temperatures are high during the day. While I did not explore this here, it would be worthy of further study, particularly in the context of temperature- 
related habitat exclusion in climate change scenarios (Wenger et al. 2011). In general it is important to consider the range of thermal requirements by organisms in predicting the effects of climate change. For example, optimal temperatures for swimming capacity may not be ideal for survival at early life stages (Clark et al. 2013).

Due to logistical constraints, I used fish from the same location, Eleuthera, to calibrate the relationship between activity, temperature, and metabolism, for application in the field in both Eleuthera and Culebra. However, there could be variation in the relationship between activity, temperature, and bonefish metabolism between the two locations, as temperature-related performance often varies with the thermal regime of the environment (Lee et al. 2003; Fangue et al. 2008; Eliason et al. 2011). More specifically, I expect bonefish in Culebra would have lower temperature specific metabolic rates than those in Eleuthera, with metabolic optima shifted higher due to the thermal regime. Future research comparing temperature specific metabolic rates would provide valuable insights into variation in bioenergetics between regions.

While ectotherms often select for temperatures that are physiologically optimal for growth and performance (Magnuson et al. 1979; Angilletta et al. 2002), thermal experience can be influenced by other ecological factors such as prey and predator density and distribution (Huey \& Slatkin 1976). The extent to which predation risk and prey availability varied in the ecosystems examined in Chapters 3 and 4 of this thesis are unknown, and are certainly an area deserving of further study. For example, if prey availability increased sufficiently on shallow fringing reefs in Puerto Rico during the 
summer months to counteract the increased energetic costs of occupying warmer water, bonefish may continue to occupy these habitats in spite of high water temperatures. However, warmer water could also lead to greater predation risk if the scope for activity is higher in bonefish predators (e.g., great barracuda) than bonefish at those temperatures. Future research could explore temperature-specific scope for activity of predators and prey, as well as temporal prey availability to determine whether predators, prey, or a combination of the two promote selective habitat use. One potential approach would be to combine extensive field sampling with dynamic energy budget modeling (Kooijman 2010) to quantitatively compare the energetic balance between temperature-related metabolic costs and energy availability in prey.

Tides are a major driver of animal movement in coastal regions, due to their impact on water depth and flow characteristics, as well as exposal of temporarily available resources in intertidal zones (Morrison et al. 2002; Krumme 2009). This is particularly true for bonefish due to their tendency to exploit shallow water resources (Murchie et al. 2013). There are also complex interactions between tide and diel period that influence animal movements in coastal regions (Krumme 2009). In an enclosed wetland, disconnected from the ocean and thus largely unaffected by the changes in water level associated with tides (Chapter 2), tide state was still a significant predictor of bonefish activity levels, with higher activity during outgoing and low tides. While this could have been a spurious finding due to the short duration of the study, this same pattern was observed in bonefish activity and energy expenditure in the wild, which was highest during outgoing and low tides (Chapter 4). However, at finer spatial scales 
(Chapter 3), bonefish activity and energy expenditure were highest during flooding and ebbing tides, which is more consistent with the concept of an energy landscape (Shepard et al. 2013). While habitat and diel period were the predominant drivers of bonefish behaviour and energy expenditure at all levels of spatial scale examined in this dissertation, tides were the third explanatory factor. Here I examined tidal influences at a rather crude scale, considering only state (i.e., low, flooding, high, ebbing). Tides are much more complex than this, as water levels vary in mean height and tidal fluxes based on the lunar cycle and seasonal variation, with complex patterns through geographical space (Le Provost et al. 1998). These variations in water depth and flow can have a significant influence on fish behaviour and drive movements between coastal habitats (Krumme 2009). Indeed, bonefish tend to move offshore to spawn around new and full moons, when tidal variations are highest (Danylchuk et al. 2011). Bonefish would be a good model for exploring how tidal variations influence movement and behaviour given their broad geographic distribution across diverse ecological conditions.

Throughout this dissertation I observed significant individual-level variation in behaviour and energetics. For example, in Chapter 2, bonefish captured in the same school exhibited high variability in activity levels, with some foraging during the day and others at night. There is increasing evidence that fish have individual-level behavioural tendencies (i.e., personalities; Reale et al. 2007; Mittlebach et al. 2013), with diverse behavioural responses to human disturbance related to the their level of boldness (e.g., Wilson et al. 2015). There is also a link between metabolism and animal behaviour, where metabolic rate, activity level, and growth rate are often correlated within 
individuals (Careau et al. 2008; Mathot \& Dingemanse 2015; Careau \& Garland 2015). This individual level consistency and between individual variation may have significant implications for ecological energetics, especially comparing energetics between ecogeographic regions as I did in Chapter 4. Individual consistency is also important in terms of vulnerability to anthropogenic disturbance (Dingemanse et al. 2010). Fisheries induced evolution involves changes in fish morphology, life history, physiology, and behaviour at the population level due to the disproportionate impacts that fisheries have on certain types of individuals, which tend to be bolder and more active, with higher metabolisms and growth rates (Law 2007; Heino \& Dieckmann 2009). It is interesting to consider that anthropogenic disturbances like fishing could explain differences in metabolism and growth rates observed across the landscape in the context of ecological energetics (Tomlinson et al. 2014).

The field of ecological energetics explores how environmental factors place energetic constraints on organisms, using a mechanistic approach to describe organism distributions, as well as how and why organismal characteristics such as growth and reproductive output vary in space in time (Tomlinson et al. 2014). Temperature, food availability and distribution, competition, and predation are often recognized as key factors that place energetic constraints in the wild (Lemon 1991; Jodice et al. 2003; Brown et al. 2004). The energetic costs of movement may also be highly relevant (Shepard et al. 2013). Ultimately, the ability of animals to allocate energy to growth and reproduction depends on the ratio of energy gain to expenditure to generate surplus. Indeed, Wilson et al. (2012) showed that imperial cormorants (Phalacrocorax atriceps) 
balance the energetic costs of travelling further from the colony with increased foraging durations. The energy landscapes framework focuses on explaining movement ecology based on energy dynamics (Shepard et al. 2013). There are some examples in fish, mainly in relation to water flow characteristics in lotic environments (Hinch \& Rand 1998; McElroy et al. 2012; Burnett et al. 2014). However, in order to connect habitat or ecosystem conditions with individual and population characteristics such as growth rates, we must consider that balance of energy expenditure to gain. For example, it may be more energetically costly to move through softer substrates or rugose terrain (Wilson et al. 1991; Wall et al. 2006), but if the density of resources and predators were such that extensive movement is unnecessary, the energetic costs of occupying that habitat would be less inflated. Moreover, if complex terrains also contain high densities of prey, as in coral reefs (Pamatmat 1968; Hatcher 1990; Moberg \& Folke 1999), the energetic costs may be countered by energy gain. Shepard et al. (2013) recognize that the level of animal movement and associated energetic costs is ultimately related to the distribution of resources as well as the locomotory capabilities of the animals, citing examples in birds and flying insects (Reynolds \& Reynolds 2009; Chapman et al. 2010). Whether or not the entire energetic interaction between animals and their ecosystems could be considered an energy landscape, or simply fall within the realm of ecological energetics, may be the subject of further philosophical discourse. Elements of my research in Chapters 2-4 could also be considered in the realm of macrophysiology (Chown \& Gaston 2016), functional biogeography (Violle et al. 2014), or in a more applied context (e.g., climate change), conservation physiology (Cooke et al. 2013b). 
In Chapters 3 and 4 I aimed to explore the ecological energetics of bonefish by examining energy expenditure in relation to landscape characteristics, without quantifying the ratio of effort (activity) to movement as per a true energy landscape (Shepard et al. 2013). Future research could explore the energy landscape for bonefish with the fine scale positioning system in Culebra (or other species with similar tracking systems), by examining movement paths in relation to activity and energy expenditure. In particular, while near-shore marine environments do not have consistent flow patterns like lotic environments, tides do generate periodic fluctuations in water flow that may further describe variation in energy expenditure, influencing the energy landscape for animals in these ecosystems. In Chapter 3 bonefish energy expenditure was highest during flowing tides (i.e., flooding and ebbing), which may be related to the energetic costs of movement through tidal water flow. However, at broader spatial scales energy expenditure was highest at ebbing and low tides (Chapter 4), and variation in bonefish energy expenditure was generally low across tidal periods in comparison to other factors (i.e., habitat, diel period).

Acceleration- and temperature-based estimates indicated higher bonefish energy expenditure in the fringing reefs of tropical Culebra than the expansive sand flats and tidal creeks of sub-tropical Eleuthera due to a combination of higher activity levels and water temperatures (Chapter 4). However, bonefish home range sizes were significantly larger in Eleuthera than Culebra, which certainly has energetic costs that were not captured by our nodes of acoustic receivers and hence missed in our estimates of energy expenditure. It is therefore unclear from my findings which environment incurs greater 
environmental costs, as it is difficult to estimate the costs of traversing larger home ranges. While fish are ideal model species for many reasons discussed in the introductory chapter of this dissertation, our abilities to study fish in the wild are also limited by the fact that most fish are fully aquatic; these environments are hostile humans, and many technologies such as geographic positioning systems function poorly (Cooke et al. 2004). Continuous tracking with acoustic telemetry is extremely challenging given the number of receivers required to cover expansive aquatic ecosystems (Donaldson et al. 2014). Biologgers are an ideal alternative, except that recapturing tagged fish is often a proverbial needle in a haystack (Brown et al. 2013). However, time releases enabling loggers to pop off fish to be recovered via radio signals at the surface are a growing alternative, but require large tagging packages that currently limit applications to larger animals.

There is an intrinsic inter-relationship between water temperature, fish activity level, and energy expenditure (Halsey et al. 2015), which cumulatively resulted in higher bonefish energy expenditure estimates in tropical Culebra than sub-tropical Eleuthera (Chapter 4). This fits well with the pace of life hypothesis, which suggests that ectothermic animals such as fish generally have faster growth rates, maturation rates, and shorter life spans at warmer temperatures (Angilletta et al. 2004). However, there is currently insufficient data on bonefish life history traits, more specifically maturation rates and life spans, across the two ecogeographic regions examined in Chapter 4 to determine whether this is a pace of life phenomenon being observed. Differences in growth rates may also be related to the energetic costs of traversing a large home range, 
which is presumably necessary to exploit more dispersed prey (Charnov 1976; Pyke 1984). Future research quantifying bonefish life history traits more extensively across the ecogeographic regions bonefish occupy, as well as the density and distribution of bonefish prey may lend clues to this question.

In this dissertation I quantified bonefish energy expenditure in terms of the metabolic costs of activity (i.e., active metabolism). Another important aspect of organismal energy expenditure is the costs of digestion (i.e., specific dynamic action; SDA), which can have significant metabolic costs that last for extended periods postfeeding (Jobling 1980; Clark et al. 2010b). SDA is clearly an important consideration for quantifying the ecological energetics of wild animals, with implications for both behavioural ecology as well as bioenergetics modeling. Heart rate loggers or transmitters can be used to quantify metabolism, integrating both activity and SDA in the wild (Cooke et al. 2016). A potential approach to measuring the relative contribution of both active metabolism and SDA in the field would involve a combination of accelerometers to measure activity, as well as heart rate loggers to capture non-activity related energy expenditure caused by SDA or other factors such as predator cues (Clarke et al. 2010a; Hall \& Clark 2016). Additionally, fish sex and reproductive status are also relevant factors influencing bonefish metabolic rate and field energetics. Females experience different metabolic demands then males, often growing larger, living longer, and contributing significantly more energy to reproduction (Tytler \& Calow 1985). Characterizing sex-specific metabolic rates is likely important for accurately scaling 
energetics to the population scale or predicting the impacts of relevant stressors or climate change.

\subsection{Anthropogenic impacts on behaviour}

The nature of human-wildlife interactions is very complex, with a diversity of human activities that affect wildlife, as well as species-, population-, and individual-level responses, all mediated by environmental conditions (Rosell \& Llimona 2012). In the context of recreational fisheries, hundreds of studies have focused on how various angling tactics, environmental conditions, and fish characteristics influence the impacts of this activity on fish welfare and survival (Reviewed by Cooke \& Suski 2005; Pelletier et al. 2007; Brownscombe et al. 2016a). Interestingly, the same environmental factors I found to be significant drivers of bonefish behaviour and energetics in Chapters 2-4 of this dissertation - habitat, diel period, and temperature, are also important factors affecting the outcome of an angling event (Cooke \& Philipp 2004; Pelletier et al. 2007; Gingerich et al. 2007).

In Chapters 5 and 6 I explored how various degrees of angling stress (which would be mediated by angler behaviour and gear in real scenarios) influenced postrelease behaviour and predation risk, with the goal of understanding the behavioural mechanisms that contribute to post release predation, and potential methods to remediate this issue. In Chapter 5 I found that retaining bonefish for a short period results in improved locomotory capabilities during the time period when post-release predation risk 
is highest (Cooke \& Philipp 2004; Danylchuk et al. 2007a). This certainly has conservation implications, as it identifies a method with strong potential for reducing post-release mortality, which is a major issue for this species. Perhaps more interestingly, I also found that retained bonefish utilized shallow, nearshore areas to rest more often than immediately released fish, which tended to swim into deep water where predation risk was higher. This suggested to me that cognitive impairment (i.e., the ability of fish to perceive their environment and make behavioural choices that reduce predation risk) might also be a significant but unrecognized factor in this context. Indeed, cognitive impairment can be significant due to acute or chronic stressors (Wendelaar Bonga 1997; de Kloet 1999). It is for this reason that I distinguish locomotory and behavioural impairment in Chapter 5. To follow up on this theory, in Chapter 6 I examined the postrelease behaviour of subadult great barracuda in an enclosed wetland mesocosm, and found that fish exposed to intermediate levels of stress had similar locomotory abilities to controls, yet they swam more non-directionally and could not orient themselves to refuge as quickly. Cooke et al. (2014b) also found evidence that cognitive impairment impacts refuge use in a coral reef fish; the Spanish flag snapper (Lutjanus carponotatus). Potential cognitive mechanisms are impaired sensory abilities (i.e., visual, olfactory, somatosensory), or cognitive abilities (Braithwaite 2006; Starcke \& Brand 2012), related to metabolic stress and the accumulation of lactate (Herbert 2002), or ammonia (Walsh et al. 2007).

If cognitive impairment plays a major role in post release behaviours, this has important applied implications; for example, it explains why releasing angled bonefish 
near refuge does not reduce predation risk (Danylchuk et al. 2007b), because they do not have the cognitive abilities to use the refuge effectively. It is notable from Chapter 6 that cognitive impairment occurs even at moderate levels of stress, in fish that have not been air exposed. Most scientists and managers recommend avoiding air exposure when practicing catch-and-release to improve fish survival (Pelletier et al. 2007). Reflex tests, which generally reflect the level of 'behavioural' (locomotory) impairment (Davis 2007, 2010; Raby et al. 2012), tend to find little to no reflex impairment in recreational angling scenarios when air exposure is avoided (Brownscombe et al. 2014, 2015). However, cognitive impairment may still be present. In angling scenarios where post-release predation risk is high, it may be advisable to retain fish for a short period to enable recovery of cognitive abilities prior to release, even when fish do not appear impaired by the standard of reflex tests. It is important to consider that retaining fish can cause increased physiological stress, although this is dependent on the method of retention. For example, retaining physiologically stressed Pacific salmon (Oncorhynchus spp.) in recovery bags similar to those used in Chapter 5 conferred some physiological and survival benefits (Donaldson et al. 2013), however, recovery by direct handling actually had a negative effect (Robinson et al. 2015). Indeed, recent research showed that manual recovery methods involving maneuvering fish through the water by hand conferred no benefits to physiological or behavioural recovery (Brownscombe et al. 2016b). 


\subsection{Summary}

Through the research in my dissertation I have found much support for my hypothesis that ecological and anthropogenic factors influence animal behaviour and energetics. For bonefish, landscape features had the greatest influence on behaviour and energy expenditure at multiple spatial scales, followed by diel period, temperature, and tide. Understanding the impacts of landscape features and anthropogenic activities on the animal energy dynamics provides mechanistic insight into how and why animals are distributed through space and time, and enables predictions for the future (Tomlinson et al. 2014). It will be important to also integrate measures of physiological stress into the landscape ecology framework to make predictions for the future impacts of anthropogenic change (Ellis et al. 2012). There is much research to be done on this topic to further understand how ecological factors influence individual and population characteristics such as behaviour and growth, as well as how ecological energetics vary for different types of species and ecogeographic regions. In particular, exploration of the influences of the spatiotemporal distribution of resources and predation risk on animal energy expenditure would be novel, along with concurrent observation of fish energy expenditure, space use, and growth. I observed significant variability in behaviour and energetics between individuals in this dissertation, and further examination of the underlying intrinsic (e.g., sex, organ size, aerobic enzymatic capacity) and extrinsic (e.g., habitat use, thermal experience, predation risk) factors, as well as correlations between characteristics (e.g. activity levels, foraging rates, home range size), would yield valuable insights for fundamental and applied ecology. Given that activity rates can be highly 
variable in the wild, and significant a driver of energy expenditure in fish (Boisclair \& Leggett 1989) estimates of field metabolic rate are also important for accurate bioenergetics models (Brodie et al. 2016). Regarding anthropogenic impacts, acute stressors (simulated angling - exercise and/or air exposure) caused both locomotory and cognitive impairment, the latter of which is particularly relevant and underappreciated in the context of stress related predation risk. Future research may aim to test other methods of recovery in different angling contexts (e.g., holding in an onboard livewell), various holding durations across different levels of stressors, as well as the benefits of the holding fish in environments with greater predation risk than observed here. Overall, I hope this dissertation has advanced our understanding of fundamental ecology and applied management of coastal marine fish and their ecosystems. 


\section{Appendix A. Abstracts of non-thesis publications during doctoral studies}

1. Lennox, R. J., Brownscombe, J. W., Cooke, S. J., Danylchuk, A. J., Moro, P. S., Sanches, E. A., \& Garrone-Neto, D. 2015. Evaluation of catch-and-release angling practices for the fat snook Centropomus parallelus in a Brazilian estuary. Ocean \& Coastal Management 113:1-7.

Recreational fisheries are developing for many iconic marine and estuarine fish species and are generating important social and economic returns in Brazil. Fat snook is the primary target species in southeastern Brazil and it supports a vibrant fisheries sector, including recreational fishing tournaments. To evaluate the impacts of recreational angling practices, we examined external hooking injuries, secondary physiological stress responses (i.e., blood glucose and lactate concentrations; $\mathrm{N}=24$ ), reflex impairment $(\mathrm{N}=39)$, and overnight survival $(\mathrm{N}=39)$ of fat snook angled with and without air exposure. We also examined physiological stress and reflex impairment of fat snook captured in a fishing tournament $(\mathrm{N}=43)$ immediately post weigh-in and $1 \mathrm{~h}$ post weighin. Values were compared to baseline reflexes $(\mathrm{N}=10)$ and physiology $(\mathrm{N}=8)$ collected from minimally stressed fat snook. Fish captured by anglers infrequently had hooking injury but all angling and tournament treatments resulted in significantly elevated blood glucose and lactate, indicating a considerable physiological response to angling. Angling with air exposure and tournament weigh-in (in a dry container) resulted in the highest blood lactate values. The body flex reflex was occasionally impaired after angling or tournament practices, but this was consistent with baseline reflex actions, and the body flex reflex was therefore not a valuable indicator of fisheries stress or mortality in fat snook. Although angling and tournament practices caused a marked physiological stress response, low mortality of snook indicated that the species is resilient to brief bouts of angling at moderate water temperatures. However, high lactate values associated with air exposure suggest that handling practices be refined, particularly during tournament weigh-in. We recommend that anglers reduce air exposure intervals when handling snook 
prior to release and that recreational tournaments adopt wet weigh-in procedures to maximize the utility of catch-and-release in this fishery.

2. Bower, S. D., Danylchuk, A. J., Brownscombe, J. W., Thiem, J. D., \& Cooke, S. J. 2016. Evaluating effects of catch-and-release angling on peacock bass (Cichla ocellaris) in a Puerto Rican reservoir: A rapid assessment approach. Fisheries Research 175:95-102.

Catch-and-release recreational angling is growing in developing countries in tropical regions and can generate valuable economic activity. However, management agencies may not have the capacity for studies to determine how angling and handling practices influence the fate of released fish, an important consideration for effective management of the resource. We developed a 3-day rapid assessment protocol and used it to evaluate the consequences of recreational fishing using artificial lures to target peacock bass (Cichla ocellaris) in the La Plata reservoir, Puerto Rico. Peacock bass were angled using conventional rod and reel and exposed to either short (minimum angling times and air exposures $<45 \mathrm{~s}$ ) or extended (extended angling times and air exposures $>1 \mathrm{~min}$ ) handling practices in water temperatures averaging $30 \pm 1{ }^{\circ} \mathrm{C}$. Angling-related injuries, post-capture physiological stress (as measured by blood sampling) and reflex impairment were quantified prior to holding angled fish in floating net pens overnight to quantify mortality. Blood glucose and lactate levels were elevated post-capture, although the changes to reflex impairment were negligible. Injuries were minimal with most fish hooked in the jaw. Peacock bass exhibited high post-capture survival $(95 \%, n=97$, size range 140-495 $\mathrm{mm}$ total length) with mortality only associated with the extended handling treatment. Using a rapid assessment protocol we were able to generate useful information on the consequences of catch-and-release on a poorly studied sportfish and identify where efforts should be directed for future studies on this and similar species. 
3. Brownscombe, J. W., Griffin, L. P., Gagne, T., Haak, C. R., Cooke, S. J., \& Danylchuk, A. J. 2015. Physiological stress and reflex impairment of recreationally angled bonefish in Puerto Rico. Environmental Biology of Fishes 98:2287-2295.

Bonefish (Albula spp.) are a popular target for recreational anglers practicing primarily catch-and-release $(C \& R)$. The effects of $C \& R$ on bonefish have been previously examined in subtropical regions of The Bahamas. However, it is unclear how these findings relate to larger bonefish, as well as those caught in warmer, tropical environments. Here we examined the effects of $C \& R$ on physiological stress and reflex impairment in bonefish in tropical Culebra, Puerto Rico. Bonefish (Albula vulpes; $56.6 \pm 5.0 \mathrm{~cm}$ total length, mean $\pm \mathrm{SE}$ ) were angled with spinning or fly fishing gear and either handled entirely in the water without air exposure $(n=12)$ or exposed to air for 2 min to simulate an aerial hook removal and admiration period $(n=11)$. Upon capture, bonefish were assessed for the presence of five reflexes and held in floating net pens for $1 \mathrm{~h}$ prior to phlebotomy for quantification of physiological stress. Blood lactate and $\mathrm{pH}$ were correlated with fight time, but linear models showed air exposure and water temperature were better predictors of physiological stress. Compared to previous research using simulated angling events, larger bonefish in warmer water under true angling scenarios had similar physiological stress responses. Two minutes of air exposure caused significant reflex impairment, which is a strong predictor of post release behavioural impairment and mortality risk in this species. Air exposure should be avoided with bonefish, especially with larger fish and at warmer water temperatures to minimize negative impacts and ensure the sustainability of C\&R bonefish angling. 
4. Wilson, A. D., Brownscombe, J. W., Krause, J., Krause, S., Gutowsky, L. F., Brooks, E. J., \& Cooke, S. J. 2015. Integrating network analysis, sensor tags, and observation to understand shark ecology and behavior. Behavioral Ecology DOI: 10.1093.

Group living in animals is a well-studied phenomenon, having been documented extensively in a wide range of terrestrial, freshwater, and marine species. Although social dynamics are complex across space and time, recent technological and analytical advances enable deeper understanding of their nature and ecological implications. While for some taxa, a great deal of information is known regarding the mechanistic underpinnings of these social processes, knowledge of these mechanisms in elasmobranchs is lacking. Here, we used an integrative and novel combination of direct observation, accelerometer biologgers, and recent advances in network analysis to better understand the mechanistic bases of individual-level differences in sociality (leadership, network attributes) and diel patterns of locomotor activity in a widespread marine predator, the lemon shark (Negaprion brevirostris). We found that dynamic models of interaction based on Markov chains can accurately predict juvenile lemon shark social behavior and that lemon sharks did not occupy consistent positions within their network. Lemon sharks did however preferentially associate with specific group members, by sex as well as by similarity or nonsimilarity for a number of behavioral (nonsimilarity: leadership) and locomotor traits (similarity: proportion of time swimming "fast," mean swim duration; nonsimilarity: proportion of swimming bursts/transitions between activity states). Our study provides some of the first information on the mechanistic bases of group living and personality in sharks and further, a potential experimental approach for studying fine-scale differences in behavior and locomotor patterns in difficult-to-study organisms. 
5. Wilson, A. D., Brownscombe, J. W., Sullivan, B., Jain-Schlaepfer, S., \& Cooke, S. J. 2015. Does Angling Technique Selectively Target Fishes Based on Their Behavioural Type?. PloS one 10:e0135848.

Recently, there has been growing recognition that fish harvesting practices can have important impacts on the phenotypic distributions and diversity of natural populations through a phenomenon known as fisheries-induced evolution. Here we experimentally show that two common recreational angling techniques (active crank baits versus passive soft plastics) differentially target wild largemouth bass (Micropterus salmoides) and rock bass (Ambloplites rupestris) based on variation in their behavioural tendencies. Fish were first angled in the wild using both techniques and then brought back to the laboratory and tested for individual-level differences in common estimates of personality (refuge emergence, flight-initiation-distance, latency-to-recapture and with a net, and general activity) in an in-lake experimental arena. We found that different angling techniques appear to selectively target these species based on their boldness (as characterized by refuge emergence, a standard measure of boldness in fishes) but not other assays of personality. We also observed that body size was independently a significant predictor of personality in both species, though this varied between traits and species. Our results suggest a context-dependency for vulnerability to capture relative to behaviour in these fish species. Ascertaining the selective pressures angling practices exert on natural populations is an important area of fisheries research with significant implications for ecology, evolution, and resource management. 
6. Nowell, L. B., Brownscombe, J. W., Gutowsky, L. F., Murchie, K. J., Suski, C. D., Danylchuk, A. J., ... \& Cooke, S. J. 2015. Swimming energetics and thermal ecology of adult bonefish (Albula vulpes): a combined laboratory and field study in Eleuthera, The Bahamas. Environmental Biology of Fishes 98:2133-2146.

Knowledge of the swimming energetics and thermal ecology of sub-tropical and tropical coastal species is extremely limited, yet this information is critical for understanding animal-environment relation- ships in the face of climate change. Using the ecologically and economically important sportfish, bonefish (Albula vulpes), we determined the critical swimming speed (Ucrit), metabolic rates $\left(\mathrm{MO}_{2 \max }\right.$ and $\left.\mathrm{MO}_{2 \text { routine}}\right)$, scope for activity, and cost of transport (COTnet) across a range of temperatures using a swim tunnel. For both critical swimming speed and scope for activity, optimal (Topt) and critical (Tcrit) temperatures were determined. The optimal temperature for Ucrit (96 $\mathrm{cm} / \mathrm{s})$ was $28.0^{\circ} \mathrm{C}$ and the optimal temperature for scope for activity $(7.5 \mathrm{mgO} / \mathrm{min} / \mathrm{kg})$ was $26.7^{\circ} \mathrm{C}$. We also estimated the thermal profile of bonefish in the wild using surgically implanted thermal loggers. Of the 138 implanted fish, eight were recaptured with functional loggers. After 220 days more than $55 \%$ of recaptured tagged fish had expelled their thermal loggers. Thermal profiles revealed that bonefish did not exceed laboratory- determined critical temperatures (i.e., $14.5^{\circ} \mathrm{C}$ minima and $37.9^{\circ} \mathrm{C}$ maxima) and spent the majority of their time at their critical swimming speed optimal temperature. Nonetheless, fish experienced wide variation in daily temperature - both through time (up to $8{ }^{\circ} \mathrm{C}$ diel fluctuation and $14{ }^{\circ} \mathrm{C}$ seasonally) and among individ- uals. Collectively, laboratory and field data suggest that bonefish occupy habitats that approach, but rarely ex- ceed ( $0.51 \%$ of the time) their Tcrit. Bonefish routinely experienced water temperatures in the field that exceeded their Topt ( $\sim 54 \%$ of the time). Even minor increases in temperature (e.g., $1^{\circ} \mathrm{C}$ ) in tidal creeks will lead to greater exceedances of Topt and Tcrit or potential- ly reduce access of bonefish to essential feeding areas. 
7. Gutowsky, L. F., Aslam, W., Banisaeed, R., Bell, L. R., Bove, K. L.,

Brownscombe, J. W., ... \& Cooke, S. J. 2015. Considerations for the design and interpretation of fishing release mortality estimates. Fisheries Research 167:64-70.

To generate mortality estimates for fish that are captured and released in recreational and commercial fisheries, it is common to temporarily hold fish in captivity. Typically, captured fish are placed in some form of pen, cage or tank with control individuals, yet little is known about how the type of holding environment influences fish condition or mortality. Here we captured freshwater fish (bluegill; Lepomis macrochirus) via angling and fyke net and retained them in one of four holding environments; a round flowthrough tank on shore [TANK], a knotless nylon pen with natural substrate in the lake [PEN], a knotless nylon floating cage with a rigid structure [RCAGE], and a knotless nylon floating cage that lacked rigid structure [CAGE]. Mortality was low (1\%) across both capture techniques and holding environments during the 14-day retention period. All mortalities were associated with capture by fyke net. A chronic stress indicator, blood glucose, was determined for a subset of fish on day 5. Although there were significant differences in blood glucose between angled RCAGE and angled PEN (Tukey, $P=0.047$ ) and angled RCAGE and fyke PEN (Tukey, $P=0.015$ ), the observed levels were generally quite low (range: $1.0-3.9 \mathrm{mmol} \mathrm{L}^{-1}$ ) and the differences were likely associated with differences in feeding; fish in the PEN group with access to substrate (and presumably the most food) had slightly higher glucose levels. At the conclusion of the study Fulton's condition factor was similar among all groups (ANOVA, $P>0.05$, all terms). However, fish held in the TANK treatment had the highest levels of external protozoan parasite infection by Ichthyopthirius (Tukey, $P<0.05$ ). This study illustrates that in situ holding environments (rather than tanks) may help reduce mortality, stress, and disease during studies that estimate post-release mortality. We encourage additional research to explore how the holding environment can influence inferences made about post-release mortality and sublethal impacts of fishing. 
8. Brownscombe, J. W., Wilson, A. D., Samson, E., Nowell, L., Cooke, S. J., \& Danylchuk, A. J. 2015. Individual differences in activity and habitat selection of juvenile queen conch evaluated using acceleration biologgers. Endangered Species Research 27:181-188.

Fine-scale differences in behaviour and habitat use have important ecological implications, but have rarely been examined in marine gastropods. We used tri-axial accelerometer loggers to estimate activity levels and movement patterns of the juvenile queen conch Lobatus gigas $(\mathrm{n}=11)$ in 2 habitat types in Eleuthera, The Bahamas. In 2 manipulations in nearshore areas, queen conchs were equipped with accelerometers and released in adjacent coral rubble or sea- grass habitats. Queen conchs were located approximately every $6 \mathrm{~h}$ during daylight by snorkelling, to measure individual differences in linear distance moved, and after $24 \mathrm{~h}$ they were relocated to an alternate habitat ( $24 \mathrm{~h}$ in each habitat). We found significant inter-individual variability in activity levels, but more consistent levels of activity between the 2 habitat types within individual queen conchs. Four (36\%) of the individuals placed in seagrass moved back to the adjacent coral rubble habitat, suggesting selectivity for coral rubble. Individuals showed variable behavioural responses when relocated to the less preferable seagrass habitat, which may be related to differ- ing stress-coping styles. Our results suggest that behavioural variability between individuals may be an important factor driving movement and habitat use in queen conch and, potentially, their susceptibility to human stressors. This study provides evidence of diverse behavioural (activity) patterns and habitat selectivity in a marine gastropod and highlights the utility of accelerometer biologgers for continuously monitoring animal behaviour in the wild. 
9. Samson, E., Brownscombe, J. W., \& Cooke, S. J. (2014). Behavioural and reflex responses of mottled mojarra Eucinostomus lefroyi (Gerreidae) to cold shock exposure. Aquatic Biology 23:101-108.

Global climate change is predicted to increase incidences of abrupt declines in oceanic temperatures due to storms or upwelling. Fish occupying shallow, near-shore marine habitats may be vulnerable to mortality or sub-lethal fitness effects due to cold shock. Mottled mojarra Eucinostomus lefroyi are a ubiquitous prey species associated with subtropical and tropical coastal habitats and thus serve as a model for evaluating the consequences of cold shock events. Here, we conducted one of the first studies of cold shock on a tropical-subtropical fish species. Fish acclimated to $24^{\circ} \mathrm{C}$ were exposed to acute temperature drops to 16,18 , or $20^{\circ} \mathrm{C}$ for $1 \mathrm{~h}$. Ventilation rates were assessed every 15 min during exposure, and equilibrium loss every $5 \mathrm{~min}$. Thirty minutes after cold shock exposure, fish were placed in a circular swim flume and chased by hand until exhaustion to measure chase time and distance chased as proxies for swimming capabilities. Fish exposed to 18 and $20^{\circ} \mathrm{C}$ had significantly higher ventilation rates than those exposed to $16^{\circ} \mathrm{C}$ or controls held at $24^{\circ} \mathrm{C}$. Exposure to 16 and $18^{\circ} \mathrm{C}$ caused reflex impairment (e.g. no response to tail grabbing), while exposure to $20^{\circ} \mathrm{C}$ caused no impairment. After $30 \mathrm{~min}$ of recovery at ambient temperature, no reflex impairments were detected in any of the treatments, and the swimming capabilities of fish exposed to cold shock were similar to, or better than control fish. Our findings suggest that abrupt changes in water temperature $>4^{\circ} \mathrm{C}$ below ambient can cause behav- ioural impairments that may lead to mortality in this species. 
10. Brownscombe, J. W., Marchand, K., Tisshaw, K., Fewster, V., Groff, O., Pichette, M., ... \& Cooke, S. J. 2014. The influence of water temperature and accelerometer-determined fight intensity on physiological stress and reflex impairment of angled largemouth bass. Conservation Physiology 2:cou057.

Release of fish captured by recreational anglers is a common practice due to angler conservation ethics or compliance with fisheries regulations. As such, there is a need to understand the factors that influence mortality and sub-lethal impairments to ensure that catch-and-release angling is a sustainable practice. Longer angling times generally contribute to increased stress and mortality in fish such that reducing these times putatively reduces stress and improves survival. However, the relative importance of fight intensity (rather than simply duration) on fish condition is poorly understood. The objective of this research was to examine the effects of fight intensity on physiological stress and reflex impairment of largemouth bass (Micropterus salmoides). The largemouth bass were angled using conventional recreational fishing gear in May (water temperature $\left.\sim 12^{\circ} \mathrm{C}\right)$ and June $\left(\sim 22^{\circ} \mathrm{C}\right)$ of 2014 in Lake Opinicon, Ontario, Canada. Fight intensity was quantified using tri-axial accelerometer loggers mounted on the tips of fishing rods. Upon capture, reflex impairment measures were assessed, and fish were held for $1 \mathrm{~h}$ prior to blood sampling for measurement of physiological stress (blood glucose and lactate concentrations and $\mathrm{pH}$ ). Physiological stress values showed a negative trend with fight duration and total fight intensity, but a positive trend with average fight intensity. Water temperature emerged as the most important predictor of the stress response in largemouth bass, while fight duration and intensity were not strong predictors. Reflex impairment was minimal, but higher reflex impairment scores were associated with elevated blood glucose. Overall, the findings of this study suggest that angling for largemouth bass at colder temperatures $\left(<15^{\circ} \mathrm{C}\right)$ causes greater physiological stress than at warmer temperatures $\left(>20^{\circ} \mathrm{C}\right)$. Based on our findings, we conclude that fight intensity is likely not to be a major driver of physiological stress in this species using typical largemouth bass angling gear, owing to the relatively short fight times (i.e. $<2 \min )$. 
11. Brownscombe, J. W., Bower, S. D., Bowden, W., Nowell, L., Midwood, J. D., Johnson, N., \& Cooke, S. J. 2014. Canadian recreational fisheries: 35 years of social, biological, and economic dynamics from a national survey. Fisheries 39:251-260.

Fisheries and Oceans Canada has collected a unique, long-term data set on the social, biological, and economic dynamics of Canada's recreational fisheries. Starting in 1975, these data were collected through mail surveys to recreational anglers at 5-year intervals. A longitudinal analysis revealed that there was an average of 4.5 million licensed anglers catching an annual average of 255 million fish. Release rates were relatively high (53\% of fish released on average), with recent survey data (2010) suggesting that release rates had exceeded $60 \%$. Recreational anglers also contribute an average of $\$ 8.8$ billion each year to the Canadian economy. However, recreational angling has become less popular over time, and the average age of participants has increased. The data were also useful for characterizing Canada's fisheries, including species-specific catch and harvest. Canada is one of the few countries to collect such extensive recreational fisheries data at a national scale and to do so at regular intervals, an approach that could be modeled by other countries. 
12. Szekeres, P., Brownscombe, J. W., Cull, F., Danylchuk, A. J., Shultz, A. D., Suski, C. D., ... \& Cooke, S. J. 2014. Physiological and behavioural consequences of cold shock on bonefish (Albula vulpes) in The Bahamas. Journal of Experimental Marine Biology and Ecology 459:1-7.

The effects of cold shock on fish have rarely been assessed in sub-tropical regions despite the fact that such events can occur as a result of upwelling, storms, or other climactic events that are expected to increase as a result of global environmental change. Here, the sub-lethal physiological and behavioural consequences of cold shock on bonefish (Albula vulpes) were assessed using four treatment groups (control, handled control, $7{ }^{\circ} \mathrm{C}$ below ambient, and $14{ }^{\circ} \mathrm{C}$ below ambient conditions; $\mathrm{n}=8$ fish/treatment). The control and handled control remained at ambient temperature $\left(\sim 24^{\circ} \mathrm{C}\right)$ while other treatments were exposed to a $2 \mathrm{~h}$ cold shock. Handled controls were treated with the same handling procedures as cold shock treatments, while control fish were less disturbed. Ventilation rate and reflex indicators (e.g., equilibrium, response to stimuli) were recorded throughout the experiment, and swimming ability was evaluated $2 \mathrm{~h}$ after cold shock exposure. A blood sample was taken after the cold shock exposure and $2 \mathrm{~h}$ following the swimming assessment to quantify glucose and lactate concentrations, hematocrit and $\mathrm{pH}$ in whole blood, and cortisol titers from spun plasma. Bonefish exposed to $14{ }^{\circ} \mathrm{C}$ below ambient temperature experienced elevated lactate concentrations and high reflex impairment, with the majority of fish losing equilibrium $<30 \mathrm{~min}$ into exposure. During cold shock exposure, ventilation rates were significantly lower in the $7^{\circ} \mathrm{C}$ below ambient treatment than in the handled control. After cold shock exposure, swimming ability decreased as the magnitude of cold shock increased, however, it was also depressed in the handled control relative to unhandled controls. Exposure to $14^{\circ} \mathrm{C}$ below ambient temperatures had significant physiological and behavioural impacts on bonefish, while $7^{\circ} \mathrm{C}$ below ambient had little effect. Abrupt declines in water temperature in shallow tropical or sub-tropical seas $>7{ }^{\circ} \mathrm{C}$ due to storm events or upwelling have the potential to cause physiological and behavioural impairment that could lead to mortality in this species. 
13. Finn, J. T., Brownscombe, J. W., Haak, C. R., Cooke, S. J., Cormier, R., Gagne, T., \& Danylchuk, A. J. 2014. Applying network methods to acoustic telemetry data: Modeling the movements of tropical marine fishes. Ecological Modelling 293:139-149.

Modeling animal movements is fundamental to animal ecology as it provides the foundation for further exploration into mechanisms affecting individual and populationlevel processes. In the last few decades, biotelemetry has enabled scientists to track the movements of marine life across a variety of scales. However, the use of such technology is progressing faster than the analytical techniques for modeling movement patterns. In summer 2012, we deployed an acoustic telemetry array around Culebra, Puerto Rico, consisting of 48 remote receivers that can detect coded transmissions sent by tags implanted in fish. We surgically implanted transmitters in bonefish $(n=28)$, great barracuda $(n=2)$ and permit $(n=1)$ as part of a multi-year study. In January 2013, we downloaded over 850,000 detections from 39 receivers for 31 fish (several receivers had zero fish detections, and two receivers were not downloaded), and used that six-month data set to explore how graph theory and network analysis can be used to model the movement ecology of the tagged fish. We analyzed this data as two types of graphs. First, a bipartite graph was constructed by linking each fish with an edge weighted by the number of detections of that fish by that receiver. Bipartite graphs are not explicitly spatial, but rather represent which fish associate with which receivers. Second, spatial movement graphs for individuals were built by linking receivers (nodes) by edges with the number of times each fish moved along that edge as weights. The bipartite graph identified groups of fish visiting the same sites, and groups of sites visited by the same fish. Of the six community detection algorithms used, Multilevel, Fast-Greedy, and Walk-Trap performed best, with similar module partitions and modularity scores. All three of these algorithms produced modules (groups) that appear to reflect working hypotheses related to the coastal bathymetry, habitat types, and associated movement ecology of the tagged species. Spatial movement graphs were very different for each fish examined and reflect behavioral differences. Fish exhibited various movement patterns, 
some showing the pattern of a central place forager (bonefish), while others cruised along a territory (great barracuda and permit).

14. Thiem, J. D., Hatry, C., Brownscombe, J. W., Cull, F., Shultz, A. D., Danylchuk, A. J., \& Cooke, S. J. 2013. Evaluation of radio telemetry to study the spatial ecology of checkered puffers (Sphoeroides testudineus) in shallow tropical marine systems. Bulletin of marine science 89:559-569.

Knowledge of the spatial ecology of fishes aids in conservation management and informs the sustainable use of natural resources, although the techniques used to monitor movements are often restricted by the habitats occupied by fishes and our inability to observe them. Due to the rapid attenuation of acoustic signals in shallow $(<0.3 \mathrm{~m})$ estuarine environments, we examined the applicability for a novel use of radio telemetry to examine the spatial ecology of checkered puffers, Sphoeroides testudineus Linnaeus, 1758 , in shallow, tropical tidal creeks. External attachment of radio tags on the dorsal surface of the caudal peduncle resulted in reliable retention (100\% after $5 \mathrm{~d})$ for shortterm applications, and no differences were observed in swimming ability or standard metabolic rate between tagged individuals and untagged controls. However, external tagging resulted in significant postural differences during routine activity in comparison to untagged controls, although we demonstrate that this can be eliminated by reducing tag burden in future studies. A small sample of puffers $(n=5)$ was subsequently radio tagged and released into a shallow tidal creek whereby attempts were made to relocate individuals both visually and using radio telemetry over a short period (1 hr). Checkered puffers exhibited an ability to move the entire length of a small tidal creek (maximum mobility $389 \mathrm{~m}$ ), although using radio telemetry to monitor the movements of puffers had limited application with the techniques applied in the present study when individuals occupied water depths $>0.2 \mathrm{~m}$ and were actively swimming. With minor modifications to tags and by reducing tag burden, radio telemetry could serve as a feasible means of monitoring the movements of site-associated species in shallow tidal creeks. 


\section{References}

Adams, A., K. Guindon, A. Horodysky, T. MacDonald, R. McBride, J. Shenker, and R. Ward. 2012. Albula vulpes. The IUCN Red List of Threatened Species 2012: e.T194303A2310733

Adams, A. J., R. K. Wolfe, M. D. Tringali, E. Wallace, and G. T. Kellison. 2008. Rethinking the status of Albula spp. biology in the Caribbean and western Atlantic. In: Ault, J (ed.). Biology and Management of the World Tarpon and Bonefish Fisheries. CRC Press, Boca Raton.

Alexander, E. C. 1961. A Contribution to the Life History, Biology and Geographical Distribution of Bonefish, Albula vulpes (Linnaeus). Carlsberg Foundation, Copenhagen.

Altmann, S. A., and J. Altmann. 2003. The transformation of behaviour field studies. Animal Behaviour 65:413-423.

Anderson, K. J., and W. Jetz. 2005. The broad-scale ecology of energy expenditure of endotherms. Ecology Letters 8:310-318.

Angilletta, M. J., P. H. Niewiarowski, and C. A. Navas. 2002. The evolution of thermal physiology in ectotherms. Journal of Thermal Biology 27:249-268.

Angilletta, M. J., T. D. Steury, and M. W. Sears. 2004. Temperature, growth rate, and body size in ectotherms: fitting pieces of a life-history puzzle. Integrative and Comparative Biology 44:498-509.

Arlinghaus, R., and S. J. Cooke. 2009. Recreational fisheries: socioeconomic importance, conservation issues and management challenges. In: Hutton, J. and W. M. Adams (eds.). Recreational hunting, conservation and rural livelihoods: science and practice. WileyBlackwell Scientific Publications, Oxford, UK. pp. 39-57. 
Arlinghaus, R., S. J. Cooke, J. Lyman, D. Policansky, A. Schwab, C. Suski, S. G. Sutton, and E. B. Thorstad. 2007. Understanding the Complexity of Catch-and-Release in Recreational Fishing: An Integrative Synthesis of Global Knowledge from Historical, Ethical, Social, and Biological Perspectives. Reviews in Fisheries Science 15:75-167.

Arlinghaus, R., T. Klefoth, S. J. Cooke, A. Gingerich, and C. Suski. 2009. Physiological and behavioural consequences of catch-and-release angling on northern pike (Esox lucius L.). Fisheries Research 97:223-233.

Ault, J. S. 2008. Biology and Management of the World Tarpon and Bonefish Fisheries. CRC Press, Boca Raton, Florida.

Barthel, B., S. Cooke, C. Suski, and D. Philipp. 2003. Effects of landing net mesh type on injury and mortality in a freshwater recreational fishery. Fisheries Research 63:275-282.

Bates, D., M. Maechler, B. Bolker, S. Walker 2015. Fitting Linear Mixed-Effects Models Using lme4. Journal of Statistical Software 67:1-48.

Beale, C. M. 2007. The Behavioral Ecology of Disturbance Responses. International Journal of Comparative Psychology 20:111-120.

Bell, G. P. 1990. Birds and mammals on an insect diet: a primer on diet composition analysis in relation to ecological energetics. Studies in Avian Biology 13:416-426.

Bettinger, J. M., J. R. Tomasso Jr, and J. J. Isely. 2005. Hooking mortality and physiological responses of striped bass angled in freshwater and held in live-release tubes. North American Journal of Fisheries Management 25:1273-1280. 
Biro, P. A., M. V. Abrahams, J. R. Post, and E. A. Parkinson. 2004. Predators select against high growth rates and risk-taking behaviour in domestic trout populations.

Proceedings of the Royal Society of London B: Biological Sciences 271:2233-2237.

Blanc, R., M. Guillemain, J.-B. Mouronval, D. Desmonts, and H. Fritz. 2006. Effects of non-consumptive leisure disturbance to wildlife. Revue d Ecologie 61:117-133.

Boisclair, D., and W. Leggett. 1989. The importance of activity in bioenergetics models applied to actively foraging fishes. Canadian Journal of Fisheries and Aquatic Sciences 46:1859-1867.

Boisclair, D., and P. Sirois. 1993. Testing assumptions of fish bioenergetics models by direct estimation of growth, consumption, and activity rates. Transactions of the American Fisheries Society 122:784-796.

Boisclair, D., and M. Tang. 1993. Empirical analysis of the influence of swimming pattern on the net energetic cost of swimming in fishes. Journal of Fish Biology 42:169183.

Boujard, T., and J. F. Leatherland. 1992. Circadian rhythms and feeding time in fishes. Environmental Biology of Fishes 35:109-131.

Bradshaw, D. 2003. Vertebrate ecophysiology: an introduction to its principles and applications. Cambridge University Press, Cambridge.

Braithwaite, V., and T. B. De Perera. 2006. Short-range orientation in fish: how fish map space. Marine and Freshwater Behaviour and Physiology 39:37-47.

Breiman, L., J. Friedman, C. J. Stone, and R. A. Olshen. 1984. Classification and regression trees. CRC press. 
Brett, J. 1964. The respiratory metabolism and swimming performance of young sockeye salmon. Journal of the Fisheries Board of Canada 21:1183-1226.

Brett, J. 1979. Environmental Factors and Growth. Fish physiology 8:599-675.

Brodie, S., M. D. Taylor, J. A. Smith, I. M. Suthers, C. A. Gray, and N. L. Payne. 2016. Improving consumption rate estimates by incorporating wild activity into a bioenergetics model. Ecology and Evolution. DOI: 10.1002/ece3.2027.

Broell, F., T. Noda, S. Wright, P. Domenici, J. F. Steffensen, J. P. Auclair, and C. T. Taggart. 2013. Accelerometer tags: detecting and identifying activities in fish and the effect of sampling frequency. Journal of Experimental Biology 216:1255-1264.

Brown, D. D., R. Kays, M. Wikelski, R. P. Wilson, and A. P. Klimley. 2014. Observing the unwatchable through acceleration logging of animal behavior. Animal Biotelemetry $1: 20$.

Brown, J. H., and J. F. Gillooly. 2003. Ecological food webs: high-quality data facilitate theoretical unification. Proceedings of the National Academy of Sciences 100:1467-1468.

Brown, J. H., J. F. Gillooly, A. P. Allen, V. M. Savage, and G. B. West. 2004. Toward a metabolic theory of ecology. Ecology 85:1771-1789.

Brownscombe, J. W., A. J. Danylchuk, J. M. Chapman, L. F. G. Gutowsky, S. J. Cooke. 2016a. Best practices for catch-and-release recreational fisheries - angling tools and tactics. Fisheries Research. FISH6433.

Brownscombe, J. W., S. D. Bower, W. Bowden, L. Nowell, J. D. Midwood, N. Johnson, and S. J. Cooke. 2014. Canadian recreational fisheries: 35 years of social, biological, and economic dynamics from a national survey. Fisheries 39:251-260. 
Brownscombe, J. W., L. P. Griffin, T. Gagne, C. R. Haak, S. J. Cooke, and A. J. Danylchuk. 2015. Physiological stress and reflex impairment of recreationally angled bonefish in Puerto Rico. Environmental Biology of Fishes 98:2287-2295.

Brownscombe, J. W., T. P. Parmar, J. Almeida, E. Giesbrecht, J. Batson, X. Chen, S. Wesch, T. D. Ward, C. O'Connor, and S. J. Cooke. 2016b. The efficacy of assisted ventilation techniques for facilitating the recovery of fish that are exhausted as a result of recreational angling. Fisheries Research. FISH6433.

Bryant, D. M. 1997. Energy expenditure in wild birds. Proceedings of the Nutrition Society 56:1025-1039.

Buchholz, R. 2007. Behavioural biology: an effective and relevant conservation tool. Trends in Ecology \& Evolution 22:401-407.

Burnett, N. J., S. G. Hinch, D. C. Braun, M. T. Casselman, C. T. Middleton, S. M. Wilson, and S. J. Cooke. 2014. Burst swimming in areas of high flow: delayed consequences of anaerobiosis in wild adult sockeye salmon. Physiological and Biochemical Zoology 87:587-598.

Calenge, C. 2011. Home range estimation in R: the adehabitat HR package. Saint Benoist, France.

Calow, P. 1985. Adaptive aspects of energy allocation. In: Tytler, P, P. Calow (eds.). Fish Energetics. Springer, New York.

Careau, V., and T. Garland Jr. 2015. Energetics and behavior: many paths to understanding. Trends in Ecology \& Evolution 30:50-60.

Careau, V., D. Thomas, M. Humphries, and D. Réale. 2008. Energy metabolism and animal personality. Oikos 117:641-653. 
Caro, T. 1999. The behaviour-conservation interface. Trends in Ecology \& Evolution 14:366-369.

Caro, T. 2007. Behavior and conservation: a bridge too far? Trends in Ecology \& Evolution 22:394-400.

Castilla, J. C. 1998. Coastal marine communities: trends and perspectives from humanexclusion experiments. Trends in Ecology \& Evolution 14:280-283.

Chapman, J. W., R. L. Nesbit, L. E. Burgin, D. R. Reynolds, A. D. Smith, D. R. Middleton, and J. K. Hill. 2010. Flight orientation behaviors promote optimal migration trajectories in high-flying insects. Science 327:682-685.

Charnov, E. L. 1976. Optimal foraging, the marginal value theorem. Theoretical Population Biology 9:129-136.

Chown, S. L., and K. J. Gaston. 2016. Macrophysiology-progress and prospects. Functional Ecology 30:330-344.

Claireaux, G., and J.-P. Lagardère. 1999. Influence of temperature, oxygen and salinity on the metabolism of the European sea bass. Journal of Sea Research 42:157-168.

Clark, T. D., E. Sandblom, E., and F. Jutfelt. 2013. Aerobic scope measurements of fishes in an era of climate change: respirometry, relevance and recommendations. Journal of Experimental Biology 216:2771-2782.

Clark, T. D., E. Sandblom, S. G. Hinch, D. A. Patterson, P. B. Frappell, and A. P. Farrell. 2010a. Simultaneous biologging of heart rate and acceleration, and their relationships with energy expenditure in free-swimming sockeye salmon (Oncorhynchus nerka). Journal of Comparative Physiology B 180: 673-684. 
Clark, K. L., G. M. Ruiz, and A. H. Hines. 2003. Diel variation in predator abundance, predation risk and prey distribution in shallow-water estuarine habitats. Journal of Experimental Marine Biology and Ecology 287:37-55.

Clark, T. D., W. T. Brandt, J. Nogueira, L. E. Rodriguez, M. Price, C. J. Farwell, and B. A. Block. 2010b. Postprandial metabolism of Pacific bluefin tuna (Thunnus orientalis). Journal of Experimental Biology 213:2379-2385.

Clarke, A., and N. M. Johnston. 1999. Scaling of metabolic rate with body mass and temperature in teleost fish. Journal of Animal Ecology 68:893-905.

Cody, M. L. 1974. Optimization in Ecology. Science 183:1156-1164.

Coleman, F. C., C. C. Koenig, and L. A. Collins. 1996. Reproductive styles of shallowwater groupers (Pisces: Serranidae) in the eastern Gulf of Mexico and the consequences of fishing spawning aggregations. Environmental Biology of Fishes 47:129-141.

Colton, D. E., and W. S. Alevizon. 1983a. Feeding Ecology of Bonefish in Bahamian Waters. Transactions of the American Fisheries Society 112:178-184.

Colton, D. E., and W. S. Alevizon. 1983b. Movement patterns of bonefish, Albula vulpes, in Bahamian waters. Fisheries Bulletin 81:148-154.

Cooke, S. J., L. Sack, C. E. Franklin, A. P. Farrell, J. Beardall, M. Wikelski, and S. L. Chown. 2013. What is conservation physiology? Perspectives on an increasingly integrated and essential science. Conservation Physiology 1:cot001.

Cooke, S., M. Donaldson, C. O'connor, G. Raby, R. Arlinghaus, A. Danylchuk, K. Hanson, S. Hinch, T. Clark, and D. Patterson. 2013a. The physiological consequences of catch-and-release angling: perspectives on experimental design, interpretation, 
extrapolation and relevance to stakeholders. Fisheries Management and Ecology 20:268287.

Cooke, S., and D. Philipp. 2008. Improving the sustainability of catch-and-release bonefish (Albula spp.) fisheries: insights for anglers, guides and fisheries managers. In: Ault, J. S. (ed.). Biology and Management of the World Tarpon and Bonefish Fisheries. CRC Press, Florida. pp. 359-381.

Cooke, S., and H. Schramm. 2007. Catch-and-release science and its application to conservation and management of recreational fisheries. Fisheries Management and Ecology 14:73-79.

Cooke, S. J., D. T. Blumstein, R. Buchholz, T. Caro, E. Fernandez-Juricic, C. E. Franklin, J. Metcalfe, C. M. O'Connor, C. C. St Clair, W. J. Sutherland, and M. Wikelski. 2014a. Physiology, behavior, and conservation. Physiological and Biochemical Zoology 87:1-14.

Cooke, S. J., and I. G. Cowx. 2004. The Role of Recreational Fishing in Global Fish Crises. Bioscience 54:857.

Cooke, S. J., and I. G. Cowx. 2006. Contrasting recreational and commercial fishing: Searching for common issues to promote unified conservation of fisheries resources and aquatic environments. Biological Conservation 128:93-108.

Cooke, S. J., S. G. Hinch, M. Wikelski, R. D. Andrews, L. J. Kuchel, T. G. Wolcott, and P. J. Butler. 2004. Biotelemetry: a mechanistic approach to ecology. Trends in Ecology \& Evolution 19:334-343.

Cooke, S. J., V. Messmer, A. J. Tobin, M. S. Pratchett, and T. D. Clark. 2014b. RefugeSeeking Impairments Mirror Metabolic Recovery Following Fisheries-Related Stressors in the Spanish Flag Snapper (Lutjanus carponotatus) on the Great Barrier Reef. Physiological and Biochemical Zoology 87:136-147. 
Cooke, S. J., and D. P. Philipp. 2004. Behavior and mortality of caught-and-released bonefish (Albula spp.) in Bahamian waters with implications for a sustainable recreational fishery. Biological Conservation 118:599-607.

Cooke, S. J., D. P. Phillip, J. F. Schreer, and S. R. McKinley. 2000. Locomotory Impairment of Nesting Male Largemouth Bass Following Catch-and-Release Angling. North American Journal of Fisheries Management 20:968-977.

Cooke, S. J., J. F. Schreer, K. M. Dunmall, and D. P. Philipp. 2002. Strategies for quantifying sublethal effects of marine catch-and-release angling-insights from novel freshwater applications. American Fisheries Society Symposium 30:121-134.

Cooke, S. J., and C. D. Suski. 2005. Do we need species-specific guidelines for catchand-release recreational angling to effectively conserve diverse fishery resources? Biodiversity and Conservation 14:1195-1209.

Cowx, I. G. 2002. Recreational fisheries. In: Hart, P. B. J., Reynolds, J.D. (eds). Handbook of Fish Biology and Fisheries. Oxford. pp. 367-390.

Cooke, S. J., L. Sack, C. E. Franklin, A. P. Farrell, J. Beardall, M. Wikelski, and S. L. Chown. 2013b. What is conservation physiology? Perspectives on an increasingly integrated and essential science. Conservation Physiology 1:cot001.

Crabtree, R. E., C. W. Harnden, D. Snodgrass, and C. Stevens. 1996. Age, growth, and mortality of bonefish, Albula vulpes, from the waters of the Florida Keys. Fishery Bulletin 94:442-451.

Crabtree, R. E., D. Snodgrass, and C. W. Harnden. 1997. Maturation and reproductive seasonality in bonefishes, Albula vulpes, from the waters of the Florida keys. Fishery Bulletin 95:456-465.

Crutzen, P. J. 2006. The “anthropocene”. Springer, New York. 
Dall, S. R. X., A. I. Houston, and J. M. McNamara. 2004. The behavioural ecology of personality: consistent individual differences from an adaptive perspective. Ecology Letters 7:734-739.

Dallas, L. J., A. D. Shultz, A. J. Moody, K. A. Sloman, and A. J. Danylchuk. 2010. Chemical excretions of angled bonefish Albula vulpes and their potential use as predation cues by juvenile lemon sharks Negaprion brevirostris. Journal of Fish Biology 77:947962.

Danylchuk, A., S. Danylchuk, D. Philipp, T. L. Goldberg, S. Cooke, and J. Koppelman. 2008. Ecology and management of bonefish (Albula spp.) in the Bahamian Archipelago. In: Ault, J. (ed.). Biology and Management of the World Tarpon and Bonefish Fisheries. CRC Press, Boca Raton. pp. 79.

Danylchuk, A. J., S. J. Cooke, T. L. Goldberg, C. D. Suski, K. J. Murchie, S. E. Danylchuk, A. D. Shultz, C. R. Haak, E. J. Brooks, and A. Oronti. 2011. Aggregations and offshore movements as indicators of spawning activity of bonefish (Albula vulpes) in The Bahamas. Marine Biology 158:1981-1999.

Danylchuk, A. J., S. E. Danylchuk, S. J. Cooke, T. L. Goldberg, J. B. Koppelman, and D. P. Philipp. 2007b. Post-release mortality of bonefish (Albula vulpes) exposed to different handling practices during catch-and-release angling in South Eleuthera, Bahamas. Fisheries Management and Ecology 14:149-154.

Danylchuk, S. E., A. J. Danylchuk, S. J. Cooke, T. L. Goldberg, J. Koppelman, and D. P. Philipp. 2007a. Effects of recreational angling on the post-release behavior and predation of bonefish (Albula vulpes): The role of equilibrium status at the time of release. Journal of Experimental Marine Biology and Ecology 346:127-133.

Darwin, C. 1859. On the Origin of Species by Means of Natural Selection, or the Preservation of Favoured Races in the Struggle for Life. John Murray, London. 
Davies, R., S. Cripps, A. Nickson, and G. Porter. 2009. Defining and estimating global marine fisheries bycatch. Marine Policy 33:661-672.

Davis, M. 2005. Behaviour impairment in captured and released sablefish: ecological consequences and possible substitute measures for delayed discard mortality. Journal of Fish Biology 66:254-265.

Davis, M. 2007. Simulated fishing experiments for predicting delayed mortality rates using reflex impairment in restrained fish. ICES Journal of Marine Science 64:1535-1542.

Davis, M., and M. Ottmar. 2006. Wounding and reflex impairment may be predictors for mortality in discarded or escaped fish. Fisheries Research 82:1-6.

Davis, M. W. 2002. Key principles for understanding fish bycatch discard mortality. Canadian Journal of Fisheries and Aquatic Sciences 59:1834-1843.

Davis, M. W. 2010. Fish stress and mortality can be predicted using reflex impairment. Fish and Fisheries 11:1-11.

de Almeida, P. R., T. J. Pereira, B. R. Quintella, A. Gronningsaeter, M. J. Costa, and J. L. Costa. 2013. Testing a 3-axis accelerometer acoustic transmitter (AccelTag) on the Lusitanian toadfish. Journal of Experimental Marine Biology and Ecology 449:230-238.

de Kloet, E. R., M. S. Oitzl, and M. Joëls. 1999. Stress and cognition: are corticosteroids good or bad guys? Trends in Neurosciences 22:422-426.

De Mitcheson, Y. S., A. Cornish, M. Domeier, P. L. Colin, M. Russell, and K. C. Lindeman. 2008. A global baseline for spawning aggregations of reef fishes. Conservation Biology 22:1233-1244. 
De Sylva, D. P. 1963. Systematics and life history of the great barracuda, Sphyraena barracuda (Walbaum).

Dingemanse, N. J., A. J. Kazem, D. Réale, and J. Wright. 2010. Behavioural reaction norms: animal personality meets individual plasticity. Trends in Ecology \& Evolution 25:81-89.

Donaldson, M., G. Raby, V. Nguyen, S. Hinch, D. Patterson, A. Farrell, M. Rudd, L. Thompson, C. O'Connor, and A. Colotelo. 2013. Evaluation of a simple technique for recovering fish from capture stress: integrating physiology, biotelemetry, and social science to solve a conservation problem. Canadian Journal of Fisheries and Aquatic Sciences 70:90-100.

Donaldson, M. R., S. G. Hinch, C. D. Suski, A. T. Fisk, M. R. Heupel, and S. J. Cooke. 2014. Making connections in aquatic ecosystems with acoustic telemetry monitoring. Frontiers in Ecology and the Environment 12:565-573.

Dugatkin, L. A. 2004. Principles of animal behavior. WW Norton, New York.

Eliason, E. J., T. D. Clark, M. J. Hague, L. M. Hanson, Z. S. Gallagher, K. M. Jeffries, M. K. Gale, D. A. Patterson, S. G. Hinch, and A. P. Farrell. 2011. Differences in thermal tolerance among sockeye salmon populations. Science 332:109-112.

Elliott, J., and W. Davison. 1975. Energy equivalents of oxygen consumption in animal energetics. Oecologia 19:195-201.

Ellis, R. D., T. J. McWhorter, and M. Maron. 2012. Integrating landscape ecology and conservation physiology. Landscape Ecology 27:1-12.

Espinoza, M., T. J. Farrugia, D. M. Webber, F. Smith, and C. G. Lowe. 2011. Testing a new acoustic telemetry technique to quantify long-term, fine-scale movements of aquatic animals. Fisheries Research 108:364-371. 
Facey, D., and G. Grossman. 1992. The relationship between water velocity, energetic costs, and microhabitat use in four North American stream fishes. Hydrobiologia 239:1-6.

Fangue, N. A., M. Mandic, J. G. Richards, and P. M. Schulte. 2008. Swimming performance and energetics as a function of temperature in killifish Fundulus heteroclitus. Physiological and Biochemical Zoology 81:389-401.

FAO, Food and Agriculture Organization of the United Nations, Fisheries and Aquaculture Department. 2012. Technical guidelines for responsible recreational fisheries. Food and Agriculture Organization, Rome.

Farrell, A., P. Gallaugher, J. Fraser, D. Pike, P. Bowering, A. K. Hadwin, W. Parkhouse, and R. Routledge. 2001. Successful recovery of the physiological status of coho salmon on board a commercial gillnet vessel by means of a newly designed revival box. Canadian Journal of Fisheries and Aquatic Sciences 58:1932-1946.

Farrell, A., S. Hinch, S. Cooke, D. Patterson, G. Crossin, M. Lapointe, and M. Mathes. 2008. Pacific salmon in hot water: applying aerobic scope models and biotelemetry to predict the success of spawning migrations. Physiological and Biochemical Zoology 81:697-709.

Field, A., Miles, J., Field, Z. 2012. Discovering statistics using R. Sage Publications Inc., Thousand Oaks, California.

Føre, M., J. A. Alfredsen, and A. Gronningsater. 2011. Development of two telemetrybased systems for monitoring the feeding behaviour of Atlantic salmon (Salmo salar L.) in aquaculture sea-cages. Computers and Electronics in Agriculture 76:240-251.

Fort, J., W. P. Porter, and D. Grémillet. 2009. Thermodynamic modelling predicts energetic bottleneck for seabirds wintering in the northwest Atlantic. Journal of Experimental Biology 212:2483-2490. 
Fraser, N. H., N. B. Metcalfe, and J. E. Thorpe. 1993. Temperature-dependent switch between diurnal and nocturnal foraging in salmon. Proceedings of the Royal Society of London B: Biological Sciences 252:135-139.

Free Software Foundation. 1991. Quantum GIS Geographic Information System. Open Source Geospatial Foundation Project.

Freire, K. M., M. L. Machado, and D. Crepaldi. 2012. Overview of inland recreational fisheries in Brazil. Fisheries 37:484-494.

Freitas, C., E. M. Olsen, H. Knutsen, J. Albretsen, and E. Moland. 2015. Temperatureassociated habitat selection in a cold-water marine fish. Journal of Animal Ecology. DOI: $10.1111 / 1365-2656.12458$.

Frid, A., and L. M. Dill. 2002. Human-caused disturbance stimuli as a form of predation risk. Conservation Ecology 6:11.

Friedlander, A. M., and E. E. DeMartini. 2002. Contrasts in density, size, and biomass of reef fishes between the northwestern and the main Hawaiian islands: the effects of fishing down apex predators. Marine Ecology Progress Series 230:e264.

Fry, F., and J. Hart. 1948. The relation of temperature to oxygen consumption in the goldfish. The Biological Bulletin 94:66-77.

FWC, Florida Fish and Wildlife Conservation Commission. 2015. FWC approves barracuda conservation measures for South Florida.

Gauthier, G., J. Bédard, and Y. Bédard. 1984. Comparison of daily energy expenditure of greater snow geese between two habitats. Canadian Journal of Zoology 62:1304-1307. 
Giacomini, H. C., B. J. Shuter, and N. P. Lester. 2013. Predator bioenergetics and the prey size spectrum: Do foraging costs determine fish production? Journal of Theoretical Biology 332:249-260.

Gibson, R. N. 1986. Intertidal teleosts: life in a fluctuating environment. In: Pitcher, T. J. (ed.). The behaviour of teleost fishes. Springer, New York. pp. 388-408.

Gillooly, J. F., J. H. Brown, G. B. West, V. M. Savage, and E. L. Charnov. 2001. Effects of size and temperature on metabolic rate. Science 293:2248-2251.

Gingerich, A. J., S. J. Cooke, K. C. Hanson, M. R. Donaldson, C. T. Hasler, C. D. Suski, and R. Arlinghaus. 2007. Evaluation of the interactive effects of air exposure duration and water temperature on the condition and survival of angled and released fish. Fisheries Research 86:169-178.

Gleiss, A. C., J. J. Dale, K. N. Holland, and R. P. Wilson. 2010. Accelerating estimates of activity-specific metabolic rate in fishes: testing the applicability of acceleration dataloggers. Journal of Experimental Marine Biology and Ecology 385:85-91.

Gleiss, A. C., R. P. Wilson, and E. L. Shepard. 2011. Making overall dynamic body acceleration work: on the theory of acceleration as a proxy for energy expenditure. Methods in Ecology and Evolution 2:23-33.

Godin, J. G. 1997. Behavioural ecology of teleost fishes. Oxford University Press, New York.

Google Earth V 7.1.5.1557 (2015). Culebra, Puerto Rico.

Graeb, B. D., J. M. Dettmers, D. H. Wahl, and C. E. Cáceres. 2004. Fish size and prey availability affect growth, survival, prey selection, and foraging behavior of larval yellow perch. Transactions of the American Fisheries Society 133:504-514. 
Green, R. J., and K. Higginbottom. 2000. The effects of non-consumptive wildlife tourism on free-ranging wildlife: a review. Pacific Conservation Biology 6:183-197.

Hall, M. A., D. L. Alverson, and K. I. Metuzals. 2000. By-catch: problems and solutions. Marine Pollution Bulletin 41:204-219.

Hall, A. E., and T. D. Clark. 2016. Seeing is believing: metabolism provides insight into threat perception for a prey species of coral reef fish. Animal Behaviour 115:117-126.

Halpern, B. S., S. Walbridge, K. A. Selkoe, C. V. Kappel, F. Micheli, C. D'Agrosa, J. F. Bruno, K. S. Casey, C. Ebert, and H. E. Fox. 2008. A global map of human impact on marine ecosystems. Science 319:948-952.

Halpin, P. M. 2000. Habitat use by an intertidal salt-marsh fish: trade-offs between predation and growth. Marine Ecology Progress Series 198:203-214.

Halsey, L. G., P. Matthews, E. Rezende, L. Chauvaud, and A. A. Robson. 2015. The interactions between temperature and activity levels in driving metabolic rate: theory, with empirical validation from contrasting ectotherms. Oecologia 177:1117-1129.

Halsey, L. G., E. L. C. Shepard, and R. P. Wilson. 2011. Assessing the development and application of the accelerometry technique for estimating energy expenditure. Comparative Biochemistry and Physiology Part A: Molecular \& Integrative Physiology 158:305-314.

Hansen, M. J., D. Boisclair, S. B. Brandt, S. W. Hewett, J. F. Kitchell, M. C. Lucas, and J. J. Ney. 1993. Applications of bioenergetics models to fish ecology and management: where do we go from here? Transactions of the American Fisheries Society 122:10191030 . 
Harfmart, K. J., and S. B. Brandt. 1995. Predatory demand and impact of striped bass, bluefish, and weakfish in the Chesapeake Bay: applications of bioenergetics models. Canadian Journal of Fisheries and Aquatic Sciences 52:1667-1687.

Hatcher, B. G. 1990. Coral reef primary productivity. A hierarchy of pattern and process. Trends in Ecology \& Evolution 5:149-155.

Hayes, D. B., C. P. Ferreri, and W. W. Taylor. 1996. Linking fish habitat to their population dynamics. Canadian Journal of Fisheries and Aquatic Sciences 53:383-390.

Heino, M., and U. Dieckmann. 2009. Fisheries-induced Evolution. eLS.

Helfman, G., B. B. Collette, D. E. Facey, and B. W. Bowen. 2009. The diversity of fishes: biology, evolution, and ecology. John Wiley \& Sons.

Helfman, G. S. 1986. Fish behaviour by day, night and twilight. In Pitcher, T. J. (ed.). The behaviour of teleost fishes. Springer. pp. 366-387.

Herbert, N. A., R. M. Wells, and J. Baldwin. 2002. Correlates of choroid rete development with the metabolic potential of various tropical reef fish and the effect of strenuous exercise on visual performance. Journal of Experimental Marine Biology and Ecology 275:31-46.

Hinch, S. G., and P. S. Rand. 1998. Swim speeds and energy use of upriver-migrating sockeye salmon (Oncorhynchus nerka): role of local environment and fish characteristics. Canadian Journal of Fisheries and Aquatic Sciences 55:1821-1831.

Holmlund, C. M., and M. Hammer. 1999. Ecosystem services generated by fish populations. Ecological Economics 29:253-268. 
Houston, A. I., J. M. McNamara, and J. M. Hutchinson. 1993. General results concerning the trade-off between gaining energy and avoiding predation. Philosophical Transactions of the Royal Society B: Biological Sciences 341:375-397.

Huey, R. B., and M. Slatkin. 1976. Cost and benefits of lizard thermoregulation. Quarterly Review of Biology 363-384.

Hugie, D. M., and L. M. Dill. 1994. Fish and game: a game theoretic approach to habitat selection by predators and prey. Journal of Fish Biology 45:151-169.

Humborstad, O.-B., M. W. Davis, and S. Løkkeborg. 2009. Reflex impairment as a measure of vitality and survival potential of Atlantic cod (Gadus morhua). Fishery Bulletin 107:395-402.

Humston, R., J. S. Ault, M. F. Larkin, and J. Luo. 2005. Movements and site fidelity of the bonefish Albula vulpes in the northern Florida Keys determined by acoustic telemetry. Marine Ecology Progress Series 291:237-248.

Hussey, N. E., S. T. Kessel, K. Aarestrup, S. J. Cooke, P. D. Cowley, A. T. Fisk, R. G. Harcourt, K. N. Holland, S. J. Iverson, and J. F. Kocik. 2015. Aquatic animal telemetry: a panoramic window into the underwater world. Science 348:1255642.

Irlich, U. M., J. S. Terblanche, T. M. Blackburn, and S. L. Chown. 2009. Insect ratetemperature relationships: Environmental variation and the metabolic theory of ecology. The American Naturalist 174:819-835.

Jain, K., J. Hamilton, and A. Farrell. 1997. Use of a ramp velocity test to measure critical swimming speed in rainbow trout (Onchorhynchus mykiss). Comparative Biochemistry and Physiology Part A: Physiology 117:441-444.

Jetz, W., C. Carbone, J. Fulford, and J. H. Brown. 2004. The scaling of animal space use. Science 306:266-268. 
Jobling, M. 1995. Fish bioenergetics. Oceanographic Literature Review 9:785.

Jobling, M., P. S. Davies. 1980. Effects of feeding on metabolic rate, and the specific dynamic action in plaice, Pleuronectes platessa L. Journal of Fish Biology 16:629-638.

Jodice, P., D. Roby, R. Suryan, D. Irons, A. Kaufman, K. Turco, and G. Visser. 2003. Variation in Energy Expenditure among Black - Legged Kittiwakes: Effects of Activity - Specific Metabolic Rates and Activity Budgets. Physiological and Biochemical Zoology 76:375-388.

Johnson, A., J. Wiens, B. Milne, and T. Crist. 1992. Animal movements and population dynamics in heterogeneous landscapes. Landscape Ecology 7:63-75.

Jones, D. R., J. Kiceniuk, and O. Bamford. 1974. Evaluation of the swimming performance of several fish species from the Mackenzie River. Journal of the Fisheries Board of Canada 31:1641-1647.

Klefoth, T., A. Kobler, and R. Arlinghaus. 2008. The impact of catch-and-release angling on short-term behaviour and habitat choice of northern pike (Esox lucius L.). Hydrobiologia 601:99-110.

Kleiber, M. 1961. The fire of life. An introduction to animal energetics. The fire of life. An introduction to animal energetics.

Kooijman, S. A. L. M. 2010. Dynamic energy budget theory for metabolic organisation. Cambridge University Press.

Kramer, D. L., and M. R. Chapman. 1999. Implications of fish home range size and relocation for marine reserve function. Environmental Biology of Fishes 55:65-79. 
Krumme, U. 2009. Diel and tidal movements by fish and decapods linking tropical coastal ecosystems. In: Nagelkerken, I. (ed.). Ecological connectivity among tropical coastal ecosystems. Springer, New York. pp. 271-324.

Langerhans, R. B., C. A. Layman, A. K. Langerhans, and T. J. Dewitt. 2003. Habitat associated morphological divergence in two Neotropical fish species. Biological Journal of the Linnean Society 80:689-698.

Lantry, B. F., and D. J. Stewart. 1993. Ecological energetics of rainbow smelt in the Laurentian Great Lakes: an interlake comparison. Transactions of the American Fisheries Society 122:951-976.

Larkin, M. F. 2011. Assessment of South Florida's bonefish stock. Ph.D. Thesis, University of Miami.

Law, R. 2007. Fisheries-induced evolution: present status and future directions. Marine Ecology Progress Series 335:271-277.

Le Provost, C., F. Lyard, J. Molines, M. Genco, and F. Rabilloud. 1998. A hydrodynamic ocean tide model improved by assimilating a satellite altimeter-derived data set. Journal of Geophysical Research: Oceans 103:5513-5529.

Lee, C., A. Farrell, A. Lotto, M. MacNutt, S. Hinch, and M. Healey. 2003. The effect of temperature on swimming performance and oxygen consumption in adult sockeye (Oncorhynchus nerka) and coho (O. kisutch) salmon stocks. Journal of Experimental Biology 206:3239-3251.

Lemon, W. C. 1991. Fitness consequences of foraging behaviour in the zebra finch. Nature 352:153-155. 
Lemon, W. C. 1993. The energetics of lifetime reproductive success in the zebra finch Taeniopygia guttata. Physiological Zoology 946-963.

Letourneur, Y. 2000. Spatial and temporal variability in territoriality of a tropical benthic damselfish on a coral reef (Reunion Island). Environmental Biology of Fishes 57:377-391.

Levin, S. A. 1992. The problem of pattern and scale in ecology: the Robert H. MacArthur award lecture. Ecology 73:1943-1967.

Lewin, W. C., R. Arlinghaus, and T. Mehner. 2006. Documented and Potential Biological Impacts of Recreational Fishing: Insights for Management and Conservation. Reviews in Fisheries Science 14:305-367.

Lima, S. L. 1986. Predation risk and unpredictable feeding conditions: determinants of body mass in birds. Ecology 377-385.

Lima, S. L., and L. M. Dill. 1990. Behavioral decisions made under the risk of predation: a review and prospectus. Canadian Journal of Zoology 68:619-640.

Lima, S. L., and P. A. Zollner. 1996. Towards a behavioral ecology of ecological landscapes. Trends in Ecology \& Evolution 11:131-135.

Lind, J., and W. Cresswell. 2005. Determining the fitness consequences of antipredation behavior. Behavioral Ecology 16:945-956.

Litvaitis, J. A., J. A. Sherburne, and J. A. Bissonette. 1986. Bobcat habitat use and home range size in relation to prey density. The Journal of wildlife management 110-117.

Lotze, H. K., H. S. Lenihan, B. J. Bourque, R. H. Bradbury, R. G. Cooke, M. C. Kay, S. M. Kidwell, M. X. Kirby, C. H. Peterson, and J. B. Jackson. 2006. Depletion, 
Degradation, and Recovery Potential of Estuaries and Coastal Seas. Science 312:18061809.

Lusseau, D., and L. Bejder. 2007. The long-term consequences of short-term responses to disturbance experiences from whalewatching impact assessment. International Journal of Comparative Psychology 20.

Mace, G. M., and P. H. Harvey. 1983. Energetic constraints on home-range size. American Naturalist 120-132.

Magnuson, J. J., L. B. Crowder, and P. A. Medvick. 1979. Temperature as an ecological resource. American Zoologist 19:331-343.

Mathot, K. J., and N. J. Dingemanse. 2015. Energetics and behavior: unrequited needs and new directions. Trends in Ecology \& Evolution 30:199-206.

McElroy, B., A. DeLonay, and R. Jacobson. 2012. Optimum swimming pathways of fish spawning migrations in rivers. Ecology 93:29-34.

McIvor, C. C., and W. E. Odum. 1988. Food, predation risk, and microhabitat selection in a marsh fish assemblage. Ecology 1341-1351.

McNab, B. K. 1963. Bioenergetics and the determination of home range size. American Naturalist 133-140.

Metcalfe, N. B., N. H. Fraser, and M. D. Burns. 1999. Food availability and the nocturnal vs. diurnal foraging trade-off in juvenile salmon. Journal of Animal Ecology 68:371-381.

Milinski, M. 1986. Constraints placed by predators on feeding behaviour. In: Pitcher, T. J. (ed.). The behaviour of teleost fishes. Springer, New York. pp. 236-252. 
Milinski, M., and R. Heller. 1978. Influence of a predator on the optimal foraging behaviour of sticklebacks (Gasterosteus aculeatus L.). Nature 275:642-644.

Milner-Gulland, E. J., J. Barlow, M. W. Cadotte, P. E. Hulme, G. Kerby, and M. J. Whittingham. 2012. Ensuring applied ecology has impact. Journal of Applied Ecology 49:1-5.

Mittelbach, G. G., N. G. Ballew, M. K. Kjelvik, and D. Fraser. 2014. Fish behavioral types and their ecological consequences. Canadian Journal of Fisheries and Aquatic Sciences 71:927-944.

Moberg, F., and C. Folke. 1999. Ecological goods and services of coral reef ecosystems. Ecological Economics 29:215-233.

Morales, J. M., P. R. Moorcroft, J. Matthiopoulos, J. L. Frair, J. G. Kie, R. A. Powell, E. H. Merrill, and D. T. Haydon. 2010. Building the bridge between animal movement and population dynamics. Philosophical Transactions of the Royal Society B: Biological Sciences 365:2289-2301.

Morris, D. W. 2003. Toward an ecological synthesis: a case for habitat selection. Oecologia 136:1-13.

Morrison, M. L., B. Marcot, and W. Mannan. 2012. Wildlife-habitat relationships: concepts and applications. Island Press, Washington.

Mosser, A. A., T. Avgar, G. S. Brown, C. S. Walker, and J. M. Fryxell. 2014. Towards an energetic landscape: broad-scale accelerometry in woodland caribou. Journal of Animal Ecology 83:916-922. 
Muoneke, M. I., and W. M. Childress. 1994. Hooking mortality: a review for recreational fisheries. Reviews in Fisheries Science 2:123-156.

Murchie, K. J., S. J. Cooke, A. J. Danylchuk, S. E. Danylchuk, T. L. Goldberg, C. D. Suski, and D. P. Philipp. 2011b. Thermal biology of bonefish (Albula vulpes) in Bahamian coastal waters and tidal creeks: An integrated laboratory and field study. Journal of Thermal Biology 36:38-48.

Murchie, K. J., S. J. Cooke, A. J. Danylchuk, S. E. Danylchuk, T. L. Goldberg, C. D. Suski, and D. P. Philipp. 2013. Movement patterns of bonefish (Albula vulpes) in tidal creeks and coastal waters of Eleuthera, The Bahamas. Fisheries Research 147:404-412.

Murchie, K. J., S. J. Cooke, A. J. Danylchuk, and C. D. Suski. 2011a. Estimates of field activity and metabolic rates of bonefish (Albula vulpes) in coastal marine habitats using acoustic tri-axial accelerometer transmitters and intermittent-flow respirometry. Journal of Experimental Marine Biology and Ecology 396:147-155.

Murchie, K. J., S. E. Danylchuk, C. E. Pullen, E. Brooks, A. D. Shultz, C. D. Suski, A. J. Danylchuk, and S. J. Cooke. 2009. Strategies for the capture and transport of bonefish, Albula vulpes, from tidal creeks to a marine research laboratory for long-term holding. Aquaculture Research 40:1538-1550.

Nagy, K. A. 1987. Field metabolic rate and food requirement scaling in mammals and birds. Ecological Monographs 112-128.

Nagy, K. A., I. A. Girard, and T. K. Brown. 1999. Energetics of free-ranging mammals, reptiles, and birds. Annual Review of Nutrition 19:247-277.

Naiman, R. J. 1988. Animal influences on ecosystem dynamics. Bioscience 38:750-752. 
Nakagawa, S., H. Schielzeth, and R. B. O'Hara. 2013. A general and simple method for obtaining $\mathrm{R}^{2}$ from generalized linear mixed-effects models. Methods in Ecology and Evolution 4:133-142.

Narazaki, T., K. Sato, K. J. Abernathy, G. J. Marshall, and N. Miyazaki. 2013.

Loggerhead turtles (Caretta caretta) use vision to forage on gelatinous prey in mid-water. PLoS ONE 8:e66043.

Nathan, R., W. M. Getz, E. Revilla, M. Holyoak, R. Kadmon, D. Saltz, and P. E. Smouse. 2008. A movement ecology paradigm for unifying organismal movement research. Proceedings of the National Academy of Sciences 105:19052-19059.

Nowell, L. B., J. W. Brownscombe, L. F. Gutowsky, K. J. Murchie, C. D. Suski, A. J. Danylchuk, A. Shultz, and S. J. Cooke. 2015. Swimming energetics and thermal ecology of adult bonefish (Albula vulpes): A combined laboratory and field study in Eleuthera, The Bahamas. Environmental Biology of Fishes 98:2133-2146.

O'Connor, M. P., S. J. Kemp, S. J. Agosta, F. Hansen, A. E. Sieg, B. P. Wallace, J. N. McNair, and A. E. Dunham. 2007. Reconsidering the mechanistic basis of the metabolic theory of ecology. Oikos 116:1058-1072.

O'Toole, A., K. Murchie, C. Pullen, K. Hanson, C. Suski, A. Danylchuk, and S. Cooke. 2011. Locomotory activity and depth distribution of adult great barracuda (Sphyraena barracuda) in Bahamian coastal habitats determined using acceleration and pressure biotelemetry transmitters. Marine and Freshwater Research 61:1446-1456.

Pamatmat, M. M. 1968. Ecology and metabolism of a benthic community on an intertidal sandflat. Internationale Revue der gesamten hydrobiologie und Hydrographie 53:211-298.

Parker, R. 1985. Survival of released red snapper. progress report to south Atlantic and Gulf of Mexico fishery management councils. Charleston, SC and Tampa, FL. 
Paterson, S. E. 1998. Group occurrence of great barracuda (Sphyraena barracuda) in the Turks and Caicos Islands. Bulletin of Marine Science 63:633-638.

Payne, N. L., B. M. Gillanders, R. S. Seymour, D. M. Webber, E. P. Snelling, and J. M. Semmens. 2011. Accelerometry estimates field metabolic rate in giant Australian cuttlefish Sepia apama during breeding. Journal of Animal Ecology 80:422-430.

Pelletier, C., K. C. Hanson, and S. J. Cooke. 2007. Do Catch-and-Release Guidelines from State and Provincial Fisheries Agencies in North America Conform to Scientifically Based Best Practices? Environmental Management 39:760-773.

Pettifor, R., C. Perrins, and R. McCleery. 1988. Individual optimization of clutch size in great tits. Nature 336:160-162.

Pfeiler, E., M. A. Mendoza, and F. A. Manrique. 1988. Premetamorphic bonefish (Albula sp.) leptocephali from the Gulf of California with comments on life history.

Environmental Biology of Fishes 21:241-249.

Pimm, S. L., and P. Raven. 2000. Biodiversity: extinction by numbers. Nature 403:843845.

Pimm, S. L., G. J. Russell, J. L. Gittleman, and T. M. Brooks. 1995. The future of biodiversity. Science 269:347.

Pinheiro, J., D. Bates, S. DebRoy, and D. Sarkar. 2014. R Core Team (2014) nlme: linear and nonlinear mixed effects models. R package version 3.1-117.

Pinheiro, J. C., and D. M. Bates. 2000. Mixed-E ects Models in S and S-Plus. Statistics and Computing. Springer. 
Pörtner, H. O., and G. Lannig. 2009. Oxygen and capacity limited thermal tolerance. Fish Physiology 27:143-191.

Pyke, G. H. 1984. Optimal foraging theory: a critical review. Annual Review of Ecology and Systematics 15:523-575.

Pyke, G. H., H. R. Pulliam, and E. L. Charnov. 1977. Optimal foraging: a selective review of theory and tests. Quarterly Review of Biology 52:137-154.

Raby, G. D., M. R. Donaldson, S. G. Hinch, D. A. Patterson, A. G. Lotto, D. Robichaud, K. K. English, W. G. Willmore, A. P. Farrell, and M. W. Davis. 2012. Validation of reflex indicators for measuring vitality and predicting the delayed mortality of wild coho salmon bycatch released from fishing gears. Journal of Applied Ecology 49:90-98.

Raby, G. D., J. R. Packer, A. J. Danylchuk, and S. J. Cooke. 2014. The understudied and underappreciated role of predation in the mortality of fish released from fishing gears. Fish and Fisheries 15:489-505.

Reale, D., S. M. Reader, D. Sol, P. T. McDougall, and N. J. Dingemanse. 2007. Integrating animal temperament within ecology and evolution. Biological reviews of the Cambridge Philosophical Society 82:291-318.

Reynolds, A. M., and D. R. Reynolds. 2009. Aphid aerial density profiles are consistent with turbulent advection amplifying flight behaviours: abandoning the epithet 'passive'. Proceedings of the Royal Society of London B: Biological Sciences 276:137-143.

Ricklefs, R. E., and M. Wikelski. 2002. The physiology/life-history nexus. Trends in Ecology \& Evolution 17:462-468.

Robbins, W., V. Peddemors, and S. Kennelly. 2011. Assessment of permanent magnets and electropositive metals to reduce the line-based capture of Galapagos sharks, Carcharhinus galapagensis. Fisheries Research 109:100-106. 
Robinson, J. G., and K. H. Redford. 1991. The use and conservation of wildlife. Neotropical Wildlife Use and Conservation 3-5.

Robinson, K. A., S. G. Hinch, G. D. Raby, M. R. Donaldson, D. Robichaud, D. A. Patterson, and S. J. Cooke. 2015. Influence of postcapture ventilation assistance on migration success of adult sockeye salmon following capture and release. Transactions of the American Fisheries Society 144:693-704.

Robinson, S. K., F. R. Thompson III, T. M. Donovan, D. R. Whitehead, and J. Faaborg. 1995. Regional forest fragmentation and the nesting success of migratory birds. Science 267:1987-1990

Rosell, C., and F. Llimona. 2012. Human-wildlife interactions. Animal Biodiversity and Conservation 35:219-220.

Rosser, A. M., and S. A. Mainka. 2002. Overexploitation and species extinctions. Conservation Biology 16:584-586.

Rutz, C., and G. C. Hays. 2009. New frontiers in biologging science. Biology Letters 5:289-292.

Rypel, A. L., C. A. Layman, and D. A. Arrington. 2007. Water depth modifies relative predation risk for a motile fish taxon in Bahamian tidal creeks. Estuaries and Coasts 30:518-525.

Sakamoto, K. Q., K. Sato, M. Ishizuka, Y. Watanuki, A. Takahashi, F. Daunt, and S. Wanless. 2009. Can ethograms be automatically generated using body acceleration data from free-ranging birds? PLoS ONE 4:e5379. 
Sapir, N., N. Horvitz, M. Wikelski, R. Avissar, Y. Mahrer, and R. Nathan. 2011. Migration by soaring or flapping: numerical atmospheric simulations reveal that turbulence kinetic energy dictates bee-eater flight mode. Proceedings of the Royal Society of London B: Biological Sciences DOI: 10.1098/rspb.2011.0358.

Schlaepfer, M. A., M. C. Runge, and P. W. Sherman. 2002. Ecological and evolutionary traps. Trends in Ecology \& Evolution 17:474-480.

Shafiei, N., M. Gray, V. Viau, and S. B. Floresco. 2012. Acute stress induces selective alterations in cost/benefit decision-making. Neuropsychopharmacology 37:2194-2209.

Shepard, E. L., R. P. Wilson, W. G. Rees, E. Grundy, S. A. Lambertucci, and S. B. Vosper. 2013. Energy landscapes shape animal movement ecology. American Naturalist 182:298-312.

Shepard, E. L. C., R. P. Wilson, L. G. Halsey, F. Quintana, A. Gómez Laich, A. C. Gleiss, N. Liebsch, A. E. Myers, and B. Norman. 2008. Derivation of body motion via appropriate smoothing of acceleration data. Aquatic Biology 4:235-241.

Shorrocks, B., and I. R. Swingland. 1990. Living in a patchy environment. Oxford University Press, London.

Shultz, A. D., K. J. Murchie, C. Griffith, S. J. Cooke, A. J. Danylchuk, T. L. Goldberg, and C. D. Suski. 2011. Impacts of dissolved oxygen on the behavior and physiology of bonefish: Implications for live-release angling tournaments. Journal of Experimental Marine Biology and Ecology 402:19-26.

Sih, A. 1980. Optimal behavior: can foragers balance two conflicting demands. Science 210:1041-1043.

Sih, A. 1984. The behavioral response race between predator and prey. The American Naturalist 123:143-150. 
Sih, A., A. Bell, and J. C. Johnson. 2004. Behavioral syndromes: an ecological and evolutionary overview. Trends in Ecology \& Evolution 19:372-378.

Smith, F. 2013. Understanding HPE in the VEMCO positioning system (VPS).

Soulé, M. E. 1985. What is conservation biology? A new synthetic discipline addresses the dynamics and problems of perturbed species, communities, and ecosystems. Bioscience 35:727-734.

Stalmaster, M. V., and J. A. Gessaman. 1984. Ecological energetics and foraging behavior of overwintering bald eagles. Ecological Monographs 407-428.

Starcke, K., and M. Brand. 2012. Decision making under stress: a selective review. Neuroscience \& Biobehavioral Reviews 36:1228-1248.

Suski, C. D., S. J. Cooke, A. J. Danylchuk, C. M. O'Connor, M.-A. Gravel, T. Redpath, K. C. Hanson, A. J. Gingerich, K. J. Murchie, and S. E. Danylchuk. 2007. Physiological disturbance and recovery dynamics of bonefish (Albula vulpes), a tropical marine fish, in response to variable exercise and exposure to air. Comparative Biochemistry and Physiology Part A: Molecular \& Integrative Physiology 148:664-673.

Suski, C. D., S. S. Killen, S. J. Cooke, J. D. Kieffer, D. P. Philipp, and B. L. Tufts. 2004. Physiological significance of the weigh-in during live-release angling tournaments for largemouth bass. Transactions of the American Fisheries Society 133:1291-1303.

Suzuki, I., Y. Naito, L. P. Folkow, N. Miyazaki, and A. S. Blix. 2009. Validation of a device for accurate timing of feeding events in marine animals. Polar Biology 32:667-671. 
Thorstad, E., B. Finstad, F. Økland, R. McKinley, and R. Booth. 1997. Endurance of farmed and sea-ranched Atlantic salmon Salmo salar L. at spawning. Aquaculture Research 28:635-640.

Tillman, D. T., R. M. May, C. L. Lehman, and M. A. Nowak. 1994. Habitat destruction and the extinction debt. Letters to Nature 371:56-66.

Tinbergen, N. 1963. On aims and methods of ethology. Zeitschrift für Tierpsychologie 20:410-433.

Tinbergen, N. 2005. On aims and methods of ethology. Animal Biology 55:297-321.

Tytler, P., Calow, P. 1985. Fish energetics: new perspectives. Croom Helm Ltd., Australia.

Villareal, T., S. Hanson, S. Qualia, E. Jester, H. Granade, and R. Dickey. 2007. Petroleum production platforms as sites for the expansion of ciguatera in the northwestern Gulf of Mexico. Harmful Algae 6:253-259.

Violle, C., P. B. Reich, S. W. Pacala, B. J. Enquist, and J. Kattge. 2014. The emergence and promise of functional biogeography. Proceedings of the National Academy of Sciences 111:13690-13696.

Vitousek, P. M., H. A. Mooney, J. Lubchenco, and J. M. Melillo. 1997. Human domination of Earth's ecosystems. Science 277:494-499.

Viviant, M., A. W. Trites, D. A. S. Rosen, P. Monestiez, and C. Guinet. 2009. Prey capture attempts can be detected in Steller sea lions and other marine predators using accelerometers. Polar Biology 33:713-719. 
Wall, J., Douglas-Hamilton, I., Vollrath, F. 2006. Elephants avoid costly mountaineering. Current Biology 16:R527-R529.

Wallace, E. M. 2014. Assessing Biodiversity, Evolution, and Biogeography in Bonefishes (Albuliformes): Resolving Relationships and Aiding Management. Ph.D. Thesis, University of Minnesota.

Walpole, M. J., and C. R. Thouless. 2005. Increasing the value of wildlife through nonconsumptive use? Deconstructing the myths of ecotourism and community-based tourism in the tropics. Conservation Biology Series-Cambridge 9:122.

Walsh, P. J., C. M. Veauvy, M. D. McDonald, M. E. Pamenter, L. T. Buck, and M. P. Wilkie. 2007. Piscine insights into comparisons of anoxia tolerance, ammonia toxicity, stroke and hepatic encephalopathy. Comparative Biochemistry and Physiology Part A: Molecular \& Integrative Physiology 147:332-343.

Walter, E. D. 1971. Aspects of the ecological energetics of the wolf spider Pardosa (Lycosa) lugubris (Walckenaer). Oecologia 7:136-154.

Weiser, L. A., and N. Stamp. 1998. Combined effects of allelochemicals, prey availability, and supplemental plant material on growth of a generalist insect predator. Entomologia Experimentalis et Applicata 87:181-189.

Wendelaar Bonga, S. 1997. The stress response in fish. Physiological Reviews 77:591625

Wenger, S. J., D. J. Isaak, C. H. Luce, H. M. Neville, K. D. Fausch, J. B. Dunham, D. C. Dauwalter, M. K. Young, M. M. Elsner, and B. E. Rieman. 2011. Flow regime, temperature, and biotic interactions drive differential declines of trout species under climate change. Proceedings of the National Academy of Sciences 108:14175-14180. 
Werner, E. E., J. F. Gilliam, D. J. Hall, and G. G. Mittelbach. 1983. An experimental test of the effects of predation risk on habitat use in fish. Ecology 64:1540-1548.

West, G. B., J. H. Brown, and B. J. Enquist. 1997. A general model for the origin of allometric scaling laws in biology. Science 276:122-126.

Whitney, N. M., Y. P. Papastamatiou, K. N. Holland, and C. G. Lowe. 2007. Use of an acceleration data logger to measure diel activity patterns in captive whitetip reef sharks,Triaenodon obesus. Aquatic Living Resources 20:299-305.

Whitney, N. M., H. L. Pratt, T. C. Pratt, and J. C. Carrier. 2010. Identifying shark mating behaviour using three-dimensional acceleration loggers. Endangered Species Research 10:71-82.

Wikelski, M., L. Spinney, W. Schelsky, A. Scheuerlein, and E. Gwinner. 2003. Slow pace of life in tropical sedentary birds: a common-garden experiment on four stonechat populations from different latitudes. Proceedings of the Royal Society of London B: Biological Sciences 270:2383-2388.

Wilson, A. D., J. W. Brownscombe, B. Sullivan, S. Jain-Schlaepfer, and S. J. Cooke. 2015. Does Angling Technique Selectively Target Fishes Based on Their Behavioural Type? PLoS ONE 10:e0135848.

Wilson, R., I. Griffiths, P. Legg, M. Friswell, O. Bidder, L. Halsey, S. Lambertucci, and E. Shepard. 2013. Turn costs change the value of animal search paths. Ecology Letters 16:1145-1150.

Wilson, R. P., B. Culik, D. Adelung, N. R. Coria, and H. J. Spairani. 1991. To slide or stride: when should Adélie penguins (Pygoscelis adeliae) toboggan? Canadian Journal of Zoology 69:221-225. 
Wilson, R. P., F. Quintana, and V. J. Hobson. 2012. Construction of energy landscapes can clarify the movement and distribution of foraging animals. Proceedings of the Royal Society B 279:975-980.

Wilson, R. P., E. L. C. Shepard, and N. Liebsch. 2008. Prying into the intimate details of animal lives: use of a daily diary on animals. Endangered Species Research 4:123-137.

Wilson, S., S. Hinch, E. Eliason, A. Farrell, and S. Cooke. 2013. Calibrating acoustic acceleration transmitters for estimating energy use by wild adult Pacific salmon. Comparative Biochemistry and Physiology Part A: Molecular \& Integrative Physiology 164:491-498.

Withers, P. C. 1992. Comparative animal physiology. Fort Worth, Saunders College.

Wolf, M., and F. J. Weissing. 2012. Animal personalities: consequences for ecology and evolution. Trends in Ecology \& Evolution 27:452-461.

Wright, S., J. D. Metcalfe, S. Hetherington, and R. Wilson. 2014. Estimating activityspecific energy expenditure in a teleost fish, using accelerometer loggers. Marine Ecology Progress Series 496:19-32.

Wydoski, R. S. 1977. Relation of hooking mortality and sublethal hooking stress to quality fishery management. Catch-and-release fishing as a management tool. Humboldt State University, Arcata, California.

Zhdanova, I. V., and S. G. Reebs. 2006. Circadian rhythms in fish. Fish physiology 24:197.

Zuur, A., E. N. Ieno, N. Walker, A. A. Saveliev, and G. M. Smith. 2009. Mixed effects models and extensions in ecology with R. Springer Science \& Business Media. 
